\title{
1 Unified neural pathways that gate affective pain and 2 multisensory innate threat signals to the amygdala
}

3

4
Sukjae Joshua Kang ${ }^{1,5}$, Shijia Liu ${ }^{1,2,5}$, Mao Ye ${ }^{1,5}$, Dong-Il Kim ${ }^{1}$, Jong-Hyun Kim¹ ${ }^{1}$, Tae Gyu Oh ${ }^{3}$, Jiahang Peng $^{1,2}$, Ronald M. Evans ${ }^{3}$, Kuo-Fen Lee ${ }^{1}$, Martyn Goulding ${ }^{4}$, Sung Han ${ }^{1,2}$ *

${ }^{1}$ Peptide Biology Laboratories, The Salk Institute for Biological Studies, La Jolla, CA 92037, USA

${ }^{2}$ Section of Neurobiology, Division of Biological Sciences, University of California, San Diego, La Jolla, CA 92093, USA

${ }^{3}$ Howard Hughes Medical Institute, Gene Expression Laboratories, The Salk Institute for Biological Studies, La Jolla, CA 92037, USA

${ }^{4}$ Molecular Neurobiology Laboratories, The Salk Institute for Biological Studies, La Jolla, CA 92037, USA

${ }^{5}$ These authors contributed equally

*Correspondence to:

Sung Han, Ph.D.

Peptide Biology Laboratories,

Salk Institute for Biological Studies

10010 N. Torrey Pines Rd.

La Jolla, CA 92037, USA

Email: sunghan@salk.edu

Phone: (858) 453-4100, x1856 


\section{Abstract}

29 Perception of aversive sensory stimuli such as pain and innate threat cues is essential for animal survival.

30 The amygdala is critical for aversive sensory perception, and it has been suggested that multiple parallel

31 pathways independently relay aversive cues from each sensory modality to the amygdala. However, a

32 convergent pathway that relays multisensory aversive cues to the amygdala has not been identified. Here,

33 we report that neurons expressing calcitonin gene-related peptide (CGRP) in the parvocellular

34 subparafasicular thalamic nucleus (SPFp) are necessary and sufficient for affective-motivational pain

35 perception by forming a spino-thalamo-amygdaloid pain pathway. In addition, we find that this thalamic

36 CGRP pain pathway, together with well-known parabrachio-amygdaloid CGRP pain pathway, is critical

37 for the perception of multisensory innate threat cues. The discovery of unified pathways that collectively

38 gate aversive sensory stimuli from all sensory modalities may provide critical circuit-based insights for

39 developing therapeutic interventions for affective pain- and innate fear-related disorders. 


\section{Introduction}

Pain is a complex sensory and emotional experience caused by tissue-damaging noxious stimuli that produce immediate avoidance behavior, as well as long-lasting aversive memories so that future damage can be avoided (Julius and Basbaum, 2001; Melzack and Casey, 1968). Therefore, the perception of pain results in behavioral outcomes similar to those associated with the perception of threats. Indeed, most research on Pavlovian threat learning has used electric foot shock, a painful noxious stimulus, as a threat cue. It has also been suggested that pain and threat perceptions interact with each other (Elman and Borsook, 2018). Individuals with pain asymbolia, who have deficits in perceiving affective-motivational aspects of pain due to damage to limbic structures, show compromised perception of threats (Berthier et al., 1988; Rubins and Friedman, 1948). Alternatively, people with affective pain disorders, such as migraine and fibromyalgia, are often hypersensitive to sensory inputs and perceive normal sensory signals as aversive (Bar-Shalita et al., 2019; López-Solà et al., 2017). Therefore, it is likely that there are unified neural circuits and brain areas that process both pain-causing noxious stimuli and threat-producing aversive sensory cues (Price, 1999).

The amygdala is a key limbic structure that integrates sensory stimuli with an internal state to generate appropriate emotional responses (Janak and Tye, 2015; LeDoux, 2012, 2000). It is activated by aversive sensory stimuli, including noxious stimuli (Ren and Neugebauer, 2010; Simons et al., 2014; Veinante et al., 2013), and lesioning the amygdala greatly attenuates the perception of multimodal sensory threats (Bach et al., 2015; Blanchard and Blanchard, 1972; Dal Monte et al., 2015) and pain (Gao et al., 2004; Helmstetter, 1992; Manning and Mayer, 1995; Tanimoto et al., 2003). Therefore, the amygdala may serve as a pivotal node in integrating and unifying all threat cues from different sensory modalities, including pain-causing noxious stimuli. Recent studies suggest that aversive sensory stimuli from each sensory modality relay threat cues to the distinct brain areas including the hypothalamus and amygdala through parallel nonoverlapping pathways. These include somatosensory (Barsy et al., 2020; Choi et al., 2020; Han et al., 2015; Sato et al., 2015), visual (Salay et al., 2018; Wei et al., 2015; Zhou et al., 2019), auditory (Barsy et al., 2020), gustatory (Carter et al., 2013; Kim et al., 2017; Wang et al., 2018) and olfactory (Rosen et al., 2015; Tong et al., 2020). However, little is known about the convergent neural circuits that relay and integrate multimodal aversive sensory signals, including nociceptive stimuli, to the amygdala.

Noxious stimuli from the periphery are relayed to the brain through two ascending pain pathways, the spinoparabrachial pathway and the spino-thalamic pathway (Bushnell et al., 2013). It is a well-established idea that the spino-thalamic pathway is involved in sensory and discriminative pain perception and that the spino-parabrachial pathway is involved in the perception of affective and motivational pain. This is because the former projects to the somatosensory cortex and the latter projects to the amygdala (Basbaum et al., 2009). Among multiple areas of the amygdala, the capsular subdivision of the central nucleus of the amygdala $(\mathrm{CeC})$ is known as the nociceptive amygdala since it is activated by nociceptive stimuli and receives direct input from the parabrachial nucleus (PBN) through the spino-parabrachial pathway (Gauriau and Bernard, 2002; Neugebauer, 2015). Nevertheless, it remains unclear how the nociceptive information is relayed to other areas of the amygdala involved in pain processing, such as the amygdala-striatum transition area (AStr) (Xiu et al., 2014) and the lateral nucleus of the amygdala (LA) (Bernard et al., 1992; Thompson and Neugebauer, 2017). In particular, the LA is critically involved in pain-induced aversive learning (LeDoux, 2007; Ressler and Maren, 2019), but the detailed pain pathway that relays nociceptive information to the LA has not been fully understood. 
Although the thalamus has been implicated in sensory and discriminative pain (Dado et al., 1994; Zhang and Giesler, 2005), some thalamic nuclei, such as the ventromedial posterior thalamus (VMpo) in primates, or the triangular subdivision of the posterior thalamus (PoT) in rodents, are thought to be involved in affective and motivational pain perception by relaying nociceptive signals to the limbic areas (Craig et al., 1994, 2000; Gauriau and Bernard, 2004a; Price, 2002). However, the involvement of these nuclei in affective and motivational pain perception is still inconclusive (Willis et al., 2002), mainly because the thalamus has many small, functionally distinct nuclei without clear anatomical boundaries. Therefore, to understand the roles of the thalamus in affective pain processing, it is critical to identify genetically defined populations of thalamic neurons that directly convey spinal nociceptive input to the limbic areas, such as the amygdala.

Calcitonin gene-related peptide (CGRP) is a 37-amino acid neuropeptide produced by peripheral neurons and mediates vasodilation and nociception (Russell et al., 2014; Russo, 2015). It is also produced in the brain and plays an essential role in aversive learning and pain perception (Palmiter, 2018; Shinohara et al., 2017; Yu et al., 2009). CGRP-expressing neurons are highly clustered in two brain areas: the external lateral subdivision of the PBN (PBel) and the parvocellular subparafascicular nucleus (SPFp) (D'Hanis et al., 2007; Dobolyi et al., 2005); (Experiment 79587715, Allen Brain Atlas). Previous studies have shown that CGRP neurons in the $\mathrm{PBel}\left(\mathrm{CGRP}^{\mathrm{PBel}}\right)$ are critically involved in transmitting affective pain signals during aversive learning (Han et al., 2015) and in transmitting visceral aversive signals to the CeA (Chen et al., 2018). On the other hand, the latter is a relatively unexplored area. The SPFp is an elongated structure that extends from the anteromedial to posterolateral thalamus (D'Hanis et al., 2007). It has been speculated that CGRP neurons in the medial part of the SPFp (CGRP ${ }^{\mathrm{SPF}}$ ) may play a role in sexual behaviors (Coolen et al., 2003a, 2003b), whereas those in the posterolateral part of the CGRP ${ }^{\mathrm{SPF}}$ may be involved in emotional behaviors, based on anatomical projections to the amygdala (D'Hanis et al., 2007; LeDoux et al., 1985; Yasui et al., 1991). The neighboring thalamic nucleus, such as the posterior intralaminar nucleus (PIL), or medial subdivision of medial geniculate nucleus (MGm) have been implicated to relay noxious stimuli to the amygdala during aversive learning (Barsy et al., 2020; Shi and Davis, 1999). However, it has not been tested whether the CGRP ${ }^{\mathrm{SPF}}$ neurons are involved in conveying pain signals to the amygdala during affective-motivational pain perception. Furthermore, it is unknown to what extent CGRP ${ }^{\mathrm{PBel}}$ and CGRP ${ }^{\mathrm{SPFp}}$ neurons play similar roles in conveying aversive sensory information to the amygdala.

Here, we report that $\mathrm{CGRP}^{\mathrm{SPF}}$ neurons receive direct monosynaptic inputs from projection neurons within the dorsal horn of the spinal cord and project their axons to multiple regions within the amygdala (namely (the AStr and the LA) and to the posterior insular cortex, but not to the somatosensory cortex. These neurons are activated by multimodal nociceptive stimuli. Silencing these neurons substantially attenuates affective and motivational pain perception, and activating these neurons induces aversion and aversive memory. Furthermore, CGRP ${ }^{\mathrm{SPF}}$ neurons, together with CGRP ${ }^{\text {PBel }}$ neurons, are collectively activated by aversive sensory stimuli from all sensory modalities (visual, auditory, somatosensory, gustatory, and olfactory), and silencing these neurons attenuates the perception of all aversive sensory stimuli. Taken together, CGRP neurons within the SPFp and PBel not only form two affective pain pathways, namely the spino-thalamoamygdaloid and the spino-parabracho-amygdaloid pathways, but they also convey aversive sensory signals from all sensory modalities to the amygdala during threat perception. 


\section{Results}

\section{CGRP $^{\text {SPFp }}$ and CGRP ${ }^{\text {PBel }}$ neurons connect multiple sensory relay areas to the amygdala}

The amygdala is critically involved in the affective-motivational pain perception. However, it is not fully understood by which the nociceptive information is conveyed to the amygdala. The spinal dorsal horn projection neurons relay nociceptive signals to the brain (Basbaum et al., 2009; Todd, 2010). One example is Tacrl-expressing neurons in the spinal dorsal horn (Barik et al., 2020; Chiang et al., 2020; Choi et al., 2020; Deng et al., 2020). To identify direct spino-recipient areas in the brain, we genetically labeled only the spinal Tacrl neurons with tdTomato fluorescent protein by the triple crossing of $\mathrm{Tacr} 1^{\mathrm{Cre}}, \mathrm{Cdx} 2^{\mathrm{FlpO}}$, and Ai65 (Rosa-CAG-FrtSTOPFrt-LoxSTOPLox-tdTomato; dsTomato) mice as described previously (Bourane et al., 2015) (Figure S1A). The tdTomato-expressing cell bodies were only observed in the spinal dorsal horn (Figure S1A). Fluorescently labeled axonal terminals were observed in multiple brain areas, including the PBN, SPFp, the posterior complex of the thalamus (Po), the ventral posterolateral nucleus of the thalamus (VPL), superior colliculus (SC), periaqueductal gray (PAG), dorsal column nuclei (DCN), and ventrolateral medulla (VLM) (Figure S1B). We then asked which of these areas project to the amygdala. By searching through the Allen Mouse Connectivity Atlas (http://connectivity.brain-map.org/), we found that the SPFp and PBel project to the LA and CeA, respectively. Interestingly, CGRP neurons are found in both the SPFp and PBel, and CGRP ${ }^{\text {PBel }}$ neurons are known to play a role in affective pain perception (Han et al., 2015). Therefore, we sought to dissect and compare the roles of these CGRP circuits in processing nociceptive sensory information and relaying this information to the amygdala.

To identify regions that lie downstream of CGRP neurons in the SPFp and PBel, we injected Credependent AAVs encoding EYFP or mCherry into the SPFp and PBel of the same Calca ${ }^{\text {Cre }}$ mouse that expresses Cre-recombinase in the CGRP-expressing neurons ( Calca gene encodes CGRP), respectively (Figure 1A). Coronal slices around AP -1.1 showed an intermingled green and red expression pattern in the CeA and LA (Figure S2A). However, posterior slices (AP -1.5) revealed distinct patterns of EYFP and mCherry in the amygdala. While the CGRP ${ }^{\text {SPFp }}$ synaptic terminals were found in the AStr, LA, and medial amygdala (MEA), the CGRP ${ }^{\mathrm{PBel}}$ terminals were most abundant in the CeA, and basomedial amygdala (BMA) (Figure 1B). CGRP ${ }^{\mathrm{SPF}}$ neurons did not project to the somatosensory cortex, instead they projected to the auditory cortex and the dorsal regions of the posterior insular cortex (pIC), whereas CGRP ${ }^{\text {PBel }}$ neurons projected to the bed nuclei of the stria terminalis (BNST), ventral posteromedial nucleus of the thalamus parvicellular part (VPMpc), parasubthalamic nucleus (PSTN), and the ventral portion of the pIC (Figure S2B).

To identify upstream brain regions that directly project their axons to the CGRP ${ }^{\text {SPFp }}$, or CGRP ${ }^{\text {PBel }}$ neurons, we performed cell-type-specific monosynaptic retrograde tracing using pseudotyped rabies virus (Kim et al., 2016). We injected AAV8-hSyn-FLEX-TVA-P2A-GFP-2A-oG into the SPFp or PBel of Calca ${ }^{C r e}$ mice and then waited three weeks before injecting EnvA- $\Delta$ G-rabies-mCherry into the same region (Figure 1C). Five days after the rabies injection, mice were sacrificed to detect starter cells in both regions (Figure 1D) and mCherry-labeled direct upstream neurons in the brain and spinal cord (Figure 1E). Histological analyses revealed that CGRP ${ }^{\mathrm{SPF}}$ neurons received inputs from superficial and deep layers of the spinal dorsal horn in multiple spinal cord segments, but most abundantly from the cervical segment (Figures 1F-1H). By contrast, fewer neurons in the spinal cord project to the $\mathrm{CGRP}^{\mathrm{PBel}}$ neurons compared to those projecting to the CGRP ${ }^{\mathrm{SPF}}$ neurons (Figures $1 \mathrm{I}-1 \mathrm{~K}$ ). Other than the spinal cord, CGRP ${ }^{\mathrm{SPFp}}$ neurons received inputs from sensory relay areas including the SC, inferior colliculus (IC), vestibular nucleus (VN), and trigeminal spinal nucleus ( $\mathrm{SpV}$ ), as well as other regions such as the hypothalamus and cortex (Figures S3A, S3C, and S3E). 
the nucleus tractus solitarius (NTS), as well as other areas such as the amygdala (in particular the CeA) and the hypothalamus including the lateral hypothalamus (LHA), zona inserta (ZI), and PSTN (Figures S3B, $\mathrm{S} 3 \mathrm{D}$, and S3F). Thus, these two populations of CGRP neurons may receive multimodal sensory inputs.

Our results show that both CGRP ${ }^{\mathrm{SPFp}}$ and $\mathrm{CGRP}^{\mathrm{PBel}}$ neurons receive monosynaptic inputs from the spinal dorsal horn, other sensory-related regions, hypothalamus, and amygdala (CGRP ${ }^{\text {PBel }}$ neurons in particular). However, in terms of output patterns, CGRP ${ }^{\text {SPFp }}$ neurons project to the LA and AStr, while CGRP ${ }^{\text {PBel }}$ neurons project to the $\mathrm{CeA}$, thereby forming complementary parallel sensory pathways to the amygdala.

\section{CGRP $^{\text {SPFp }}$ and CGRP ${ }^{\text {PBel }}$ neurons are activated by multimodal nociceptive stimuli}

Next, we investigated the response of CGRP ${ }^{\text {SPFp }}$ and CGRP ${ }^{\text {PBel }}$ neurons to multimodal nociceptive stimuli, such as mechanical, thermal, and inflammatory stimuli using the fiber photometry in vivo calcium imaging (Figure 2A). AAV-DIO-GCaMP6m was injected into the SPFp or PBel of $\mathrm{Calca}^{\mathrm{Cre}}$ mice, and an optic fiber (400 $\mu \mathrm{m}, 0.37 \mathrm{NA}$ ) was implanted above the injection site (Figures $2 \mathrm{~B}$ and $2 \mathrm{C}$ ). Previous in vivo electrophysiology studies have shown that nociceptive signals can be conveyed from the spinal cord to the brain under anesthesia (Gauriau and Bernard, 2004b; Peschanski et al., 1981). Therefore, we performed the fiber photometry experiments under light anesthesia to remove other emotional and movement artifacts.

Various intensities of mechanical stimuli $(0,50,100,200$, and $300 \mathrm{~g}$ of pressure delivered by a pressure meter, not von Frey hairs) were applied for 5 seconds to the ipsi- or contra-lateral paws or tail, resulting in intensity-dependent increases in calcium signals (Area under curve analysis; A.U.C.) in both CGRP ${ }^{\text {SPFp }}$ (Figures $2 \mathrm{D}-2 \mathrm{~F}$ ) and $\mathrm{CGRP}^{\mathrm{PBel}}$ neurons (Figures 2G-2I), but there was a significantly decreased calcium response in the $\mathrm{CGRP}^{\mathrm{PBel}}$ neurons by $300 \mathrm{~g}$ stimulation which created the inverted U-shaped intensity response curve. Nevertheless, CGRP ${ }^{\text {SPFp }}$ and CGRP $^{\text {PBel }}$ neurons display different dynamics to noxious stimuli. CGRP ${ }^{\mathrm{PBel}}$ neurons reached maximum response at lower stimulation intensity compared to the CGRP $^{\text {SPFp }}$ neurons (Figures S4A and S4B), but the latter responded faster to the stimuli, which was observed by greater initial rise slope of calcium peak in the CGRP ${ }^{\mathrm{SPF}}$ neurons compared to the CGRP ${ }^{\text {PBel }}$ neurons (Figure S4C). Although contralateral stimulation evoked greater responses than the ipsilateral stimulation, both the CGRP ${ }^{\mathrm{SPF}}$ and $\mathrm{CGRP}^{\mathrm{PBel}}$ neurons were robustly activated by noxious stimuli from both sides, contrary to the conventional lateralized ascending sensory pain pathways. (Figures S4D-S4G).

Heat stimuli $\left(25,35,45\right.$, and $\left.55^{\circ} \mathrm{C}\right)$ also induced intensity-dependent calcium increases in both $\mathrm{CGRP}^{\mathrm{SPFp}}$ (Figure 2J-2L) and CGRP ${ }^{\mathrm{PBel}}$ neurons (Figure 2M-2O). The maximum calcium peak values were higher overall in CGRP ${ }^{\mathrm{PBel}}$ neurons than in CGRP ${ }^{\mathrm{SPF}}$ neurons, both for contralateral (Figure $\mathrm{S} 4 \mathrm{H}$ ) and ipsilateral stimulations (Figure S4I). However, the CGRPSPFp neurons again responded faster to $55{ }^{\circ} \mathrm{C}$ stimuli than $\mathrm{CGRP}^{\mathrm{PBel}}$ neurons (Figure S4J). Although the difference between ipsilateral and contralateral stimulation was observed in CGRP ${ }^{\mathrm{SPF}}$ neurons, these neurons were robustly activated by thermal noxious stimuli from both sides (Figures S4K-S4N). Interestingly, calcium responses induced by mechanical stimuli were much stronger than those induced by thermal stimuli in CGRPSPFp neurons; the opposite was observed for $\mathrm{CGRP}^{\mathrm{PBel}}$ neurons. These results indicate that the CGRP ${ }^{\mathrm{SPF}}$ and $\mathrm{CGRP}^{\mathrm{PBel}}$ neurons may play different roles in conveying mechanical and thermal pain.

To assess the effects of inflammatory pain, we injected $10 \mu \mathrm{l}$ of $4 \%$ formalin into the contralateral forepaw, and calcium activity was recorded with fiber photometry under light anesthesia (Figures 2P-2U). We observed increases in activity during both the initial acute pain phase (5-10 min; Figures 2Q and 2T) and the inflammatory phase (15-45 min; Figures $2 \mathrm{R}$ and $2 \mathrm{U}$ ). Activation of these neurons by formalin was confirmed by Fos immunostaining, which again showed that bilateral CGRP ${ }^{\mathrm{SPF}}$ and $\mathrm{CGRP}^{\mathrm{PBel}}$ neurons 
were activated by unilateral formalin injection with slightly higher response in the contralateral neurons (Figures S5A-S5G).

Our data indicate that multimodal nociceptive stimuli bilaterally activate CGRP ${ }^{\text {SPFp }}$ and CGRP ${ }^{\text {PBel }}$ neurons and that these two populations differentially respond to nociceptive inputs of distinct modalities. Moreover, there is a potential feedback inhibitory circuit in the CGRP ${ }^{\text {PBel }}$ neurons, as we observed activity decreases in response to $300 \mathrm{~g}$ of mechanical pain and during the acute phase of the formalin test.

\section{Pain-induced synaptic plasticity change in CGRP ${ }^{\mathrm{SPF} p}$ and CGRP ${ }^{\mathrm{PBel}}$ neurons}

As we observed that noxious stimuli activate CGRP ${ }^{\mathrm{SPF}}$ and $\mathrm{CGRP}^{\mathrm{PBel}}$ neurons, we hypothesized that pain might alter the glutamatergic synaptic strength of these neurons. We performed ex vivo electrophysiology to test pain-induced synaptic plasticity changes. To induce pain, we injected $50 \mu 1$ of $5 \%$ formalin into the upper lip. Twenty-four hours after the injection, we prepared acute brain slices that contained the SPFp (Figure 3A) or PBel (Figure 3I). We then measured the AMPA/NMDA ratio, an index of glutamatergic synaptic strength, for both $\mathrm{CGRP}^{\mathrm{SPFp}}$ and $\mathrm{CGRP}^{\mathrm{PBel}}$ neurons using the whole-cell patch-clamp recording. For both CGRP ${ }^{\mathrm{SPF}}$ (Figures 3B and 3C) and CGRP ${ }^{\mathrm{PBel}}$ neurons (Figures $3 \mathrm{~J}$ and 3K), the AMPA/NMDA ratio increased (indicating long-term potentiation) in mice treated with formalin compared to controls. No differences were observed in the paired-pulse ratio (which indicates a presynaptic mechanism) for CGRP $^{\text {SPFp }}$ neurons (Figures 3D and 3E), but an increase in this ratio was found for CGRP ${ }^{\text {PBel }}$ neurons (Figures 3L and 3M). To determine whether the AMPA current was affected, we recorded AMPA-mediated mini EPSCs (Figures 3F and 3N). mEPSC amplitude (Figure 3G), but not frequency (Figure 3H), increased in $\mathrm{CGRP}^{\mathrm{SPFp}}$ neurons of mice subjected to pain compared with controls. For CGRP ${ }^{\mathrm{PBel}}$ neurons, the pain did not affect mEPSC amplitude (Figure 3O) but decreased frequency (Figure 3P).

These data together suggest that CGRP ${ }^{\mathrm{SPF}}$ and CGRP ${ }^{\mathrm{PBel}}$ neuronal synapses differentially increase the strength of glutamatergic signaling in response to pain.

\section{The transcriptome profiling of CGRP ${ }^{\mathrm{SPF} p}$ and CGRP ${ }^{\mathrm{PBel}}$ neurons}

To further investigate whether $\mathrm{CGRP}^{\mathrm{SPF}}$ and $\mathrm{CGRP}^{\mathrm{PBel}}$ neurons exhibit specific transcriptomic profiles associated with the affective pain perception, we conducted cell-type-specific transcriptomic profiling of CGRP neurons in the SPFp and PBel regions. The $\mathrm{Calca}^{\text {CreER }}$ mouse line was crossed with the RiboTag mouse line (Sanz et al., 2009) that has a floxed allele of hemagglutinin (HA)-tagged Rpl22 gene. As a result, the HA-tagged ribosomal protein, RPL22 is Cre-dependently expressed in the CGRP-expressing neurons. After fresh brain tissues containing the SPFp or PBel region were collected and homogenized, the ribosome-associated transcriptome was captured by immunoprecipitation with anti-HA antibodyconjugated magnetic beads. Precipitated / unprecipitated total RNAs were sequenced to profile active transcriptome enriched / deenriched in CGRP ${ }^{\mathrm{SPFp}}$ and CGRP ${ }^{\mathrm{PBel}}$ neurons. RNA sequencing results revealed that the Calca gene that encodes CGRP is highly enriched in both CGRP ${ }^{\mathrm{SPFp}}$ and CGRP ${ }^{\mathrm{PBel}}$-specific transcriptome, which served as a positive control. Moreover, genes encoding neuropeptide were specifically enriched in $\mathrm{CGRP}^{\mathrm{PBel}}$ neurons (Figure 4D), and genes encoding markers for inhibitory neurons or glia were de-enriched in both regions (Figure 4E), confirming that these neurons are glutamatergic neurons. Notably, several pain-related genes, in particular those encoding membrane proteins, were enriched in both populations (Figures 4A-4C). Interestingly, genes associated with affective pain disorders, such as Scn9a, and Faah for congenital insensitivity to pain (CIP), and Cacnala for migraine (Nassar et al., 2004; van den Maagdenberg et al., 2004; Cravatt and Lichtman, 2004) are highly enriched in these neurons (Figures 4A4C). Expression of the proteins encoded by these genes ( $\mathrm{Na}_{\vee} 1.7, \mathrm{Ca}_{\vee} 2.1$, and FAAH) in the SPFp and PBel 
was confirmed via immunohistochemistry (Figures 4F-4H). These data indicate that both CGRPSPF and $\mathrm{CGRP}^{\mathrm{PBel}}$ neurons express genes involved in pain perception, further supporting our results that these neurons form ascending pain pathways.

\section{CGRP $^{\mathrm{SPF}}$ and CGRP ${ }^{\mathrm{PBel}}$ neurons are activated by multisensory innate threat stimuli} conveying sensory information from multiple sensory modalities, such as visual stimulus via the SC, auditory stimulus via the IC, gustatory stimulus via NTS, and nociceptive stimuli via the SC and SpV. We, therefore, examined whether they are also activated by multisensory innate threat stimuli. We used the fiber photometry system to measure neural activity in response to five different aversive sensory stimuli. AAVDIO-GCaMP7s was injected into the SPFp or PBel of $\mathrm{Calca}^{\mathrm{Cre}}$ mice, and an optic fiber (400 $\left.\mu \mathrm{m}, 0.37 \mathrm{NA}\right)$ was implanted above the injection site to measure calcium activity of CGRP ${ }^{\mathrm{SPFp}}$ or $\mathrm{CGRP}^{\mathrm{PBel}}$ neurons (Figures 5A and 5B). A somatosensory stimulus was first tested by applying a foot shock in a cued fear conditioning test (Figure 5C). We associated a non-aversive low-volume tone (70 dB) with the shock to minimize the tone's aversive effect (Figure S6A). For both CGRP ${ }^{\mathrm{SPF}}$ and $\mathrm{CGRP}^{\mathrm{PBel}}$ neurons, immediate increases in neural activity were detected in response to the 2-s foot shock, but not during habituation or during the cue test (when the tone was on; Figures 5D-5E). Freezing was observed during the cue test, indicating that fear memory was formed (Figures S6B and S6C). To assess the auditory threat, an intense sound $(85 \mathrm{~dB})$ was delivered for $2 \mathrm{~s}$ (Figure 5F). Time-locked calcium responses were detected at the onset of an $85-\mathrm{dB}$ intense sound, but not a $70-\mathrm{dB}$ sound for both CGRP ${ }^{\mathrm{SPF}}$ (Figure 5G) and CGRP ${ }^{\mathrm{PBel}}$ neurons (Figure 5H). For an innate visual stimulus, a 2-s looming stimulus was given three times with 10-s intervals. Both CGRP $^{\text {SPFp }}$ (Figure 5J) and CGRP ${ }^{\text {PBel }}$ neurons (Figure 5K) displayed an increase of activity in response to the looming (large disk) compared to the control (small disk) stimulus. As an innate olfactory stimulus test, we exposed mice to a cotton swab soaked with trimethylthiazoline (TMT; Figure 5L). CGRP ${ }^{\text {SPFp }}$ neurons did not respond to TMT (Figure 5M), whereas CGRP ${ }^{\text {PBel }}$ neurons exhibited a slight increase in activity (Figure $5 \mathrm{~N}$ ). Finally, a gustatory stimulus was administered by exposing mice to quinine (vs. water; Figure 5O). When overnight water restricted mice licked quinine solution $(0.5 \mathrm{mM}), \mathrm{CGRP}^{\mathrm{SPFp}}$ neurons did not respond, compared with water controls (Figure 5P), but $\mathrm{CGRP}^{\mathrm{PBel}}$ neurons exhibited an increase in calcium activity (Figure 5Q). The calcium peak amplitude analysis shows that the calcium responses between the CGRP ${ }^{\mathrm{SPF}}$ and $\mathrm{CGRP}^{\mathrm{PBel}}$ neurons were not significantly different by somatosensory and auditory stimuli (Figures S7A and S7B). However, the CGRP ${ }^{\text {SPFp }}$ neurons showed a greater response to the visual stimulus compared to the $\mathrm{CGRP}^{\mathrm{PBel}}$ neurons (Figure S7C). In contrast, the CGRP ${ }^{\text {PBel }}$ neurons showed greater responses to the olfactory and gustatory stimuli compared to the CGRP ${ }^{\text {SPFp }}$ neurons (Figures S7D and S7E).

Our results indicate that the CGRP ${ }^{S P F p}$ and CGRP ${ }^{\text {PBel }}$ neurons are both involved in the perception of innate multisensory threat but respond differently to inputs from distinct modalities. CGRP ${ }^{\mathrm{PBel}}$ neurons were activated by all five aversive sensory stimuli, whereas the CGRP ${ }^{\text {SPFp }}$ neurons were only activated by somatosensory, auditory, and visual aversive stimuli.

\section{Silencing CGRP neurons attenuates responses to multimodal threat stimuli}

299 Our rabies tracing and fiber photometry results imply that CGRP ${ }^{\mathrm{SPF}}$ and CGRP ${ }^{\mathrm{PBel}}$ neurons are critically 300 involved in innate threat perception. Thus, we next tested whether these neurons are necessary for innate 301 threat perception. We silenced these neurons by bilateral injection of AAV-DIO-TetTox::GFP into the 
threat stimuli (Figure 6A). We first performed the formalin assay to test the affective pain perception (Figure 6B). Following injection of $4 \%$ formalin into the forepaw, mice in which CGRP ${ }^{\mathrm{SPF}}$ or CGRP $\mathrm{CBel}^{\mathrm{PBel}}$ neurons were silenced spent less time licking the injected paw (Figure 6C). In addition, the CGRP ${ }^{\text {SPFp }}$ silenced group exhibited decreased thermal sensitivity in the $55{ }^{\circ} \mathrm{C}$ hot plate test (Figure S8A and S8B), decreased mechanical sensitivity in the electronic von Frey test (Figures S8C and S8D), and decreased freezing in response to the contextual fear conditioning test (Figures S8E and S8F). Interestingly, previous

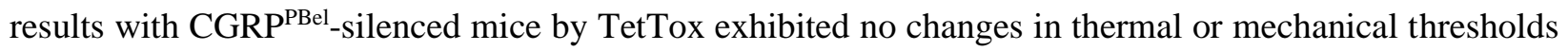
but decreased freezing in the fear conditioning test (Han et al., 2015), suggesting that both CGRP ${ }^{\mathrm{SPF}}$ and $\mathrm{CGRP}^{\mathrm{PBel}}$ neurons are necessary for affective pain perception. However, CGRP ${ }^{\mathrm{SPF}}$ neurons, not CGRP ${ }^{\text {PBel }}$ neurons, are also necessary for sensory pain perception. The elevated plus maze (EPM) test shows that silencing the CGRP ${ }^{\mathrm{SPF}}$ or CGRP ${ }^{\mathrm{PBel}}$ neurons decreased anxiety-like behaviors in mice (Figures S8G and $\mathrm{S} 8 \mathrm{H})$.

To test the role of these neurons in multisensory threat perception, the same group of mice were subjected to multiple aversive sensory threat cues, as described in Figure 5. Levels of immediate freezing in response to the aversive somatosensory stimulus (2-s, $0.6 \mathrm{~mA}$ electric foot shock) were significantly reduced in both the CGRP ${ }^{\mathrm{SPF}}$ and $\mathrm{CGRP}^{\mathrm{PBel}}$ TetTox groups compared to the EYFP control groups (Figure 6D). In the auditory threat test with $85-\mathrm{dB}$ intense sound, EYFP control mice displayed freezing behavior, but freezing levels were reduced in both the CGRP ${ }^{\mathrm{SPF}}$ and CGRP ${ }^{\mathrm{PBel}}$ TetTox groups (Figure 6E). Defensive behaviors (freezing) were also attenuated in response to a looming visual stimulus in the CGRP ${ }^{\text {SPFp }}$ TetTox group compared with controls, but no difference in freezing was observed between the CGRP ${ }^{\mathrm{PBel}}$ TetTox group and controls (Figure 6F). Interestingly, CGRP ${ }^{\mathrm{PBel}}$ neurons were activated by looming (Figure 5K), but their silencing was not enough to attenuate the animals' response to a visual threat, indicating that they play a less significant role in transmitting aversive visual stimulus to the amygdala compared to the CGRP ${ }^{\mathrm{SPF}}$ neurons. The aversive olfactory test was performed using a two-chamber system, with one chamber containing water-soaked cotton and the other containing TMT-soaked cotton. Silencing the CGRP ${ }^{\text {SPFp }}$ neurons did not affect the perception of the aversive olfactory cue, as these mice and EYFP controls both avoided the TMT chamber, while the CGRP ${ }^{\text {PBel }}$ TetTox group exhibited no aversion to TMT, spending equal amounts of time in the water and TMT chambers (Figure 6G). The gustatory test was performed as a two-bottle choice test between water and quinine solution. The CGRPSPFp TetTox group consumed minimal quinine solution $(0.5 \mathrm{mM})$, like controls, whereas the CGRP ${ }^{\mathrm{PBel}}$ TetTox group showed much less aversion to quinine (Figure $6 \mathrm{H}$ ).

These results indicate that both the CGRP ${ }^{\mathrm{SPFp}}$ and CGRP ${ }^{\mathrm{PBel}}$ neurons are necessary for the perception of innate sensory threat cues, as well as affective pain.

\section{Activating CGRP $^{\mathrm{SPF}} / \mathrm{CGRP}^{\mathrm{PBel}}$ to amygdala pathways induces negative valence}

Next, we performed optogenetic gain-of-function experiments to test whether activation of these neurons is sufficient to induce negative affect in mice. We bilaterally injected AAV-DIO-ChR2 into the SPFp of $\mathrm{Calca}^{\mathrm{Cre}}$ mice and implanted optic fibers $(200 \mu \mathrm{m}$, NA 0.22) above the injection site (Figure 7A). $20-\mathrm{Hz}$ photo-stimulation of CGRP ${ }^{\mathrm{SPF}}$ neurons did not change responses in the hot plate thermal sensitivity test and the electronic von Frey mechanical threshold test for sensory and discriminative pain perception (Figures S8I-S8L). To test whether these neurons encode negative valence, we performed the real-time place aversion (RTPA) test. Optogenetic stimulation is delivered only when the test mouse stays on one side of a two-chamber apparatus (Stamatakis and Stuber, 2012; Figure 7B). Optogenetic activation of the CGRP $^{\text {SPFp }}$ neurons induced aversion to the photo-stimulated chamber, suggesting that these neurons play a 
role in negative emotion or affective-motivational pain (Figures 7B and 7C). Next, we replaced the foot shock with photo-stimulation $(20 \mathrm{~Hz})$ as the unconditioned stimulus (US) in the context and cued fear conditioning test. This was to assess whether activation of CGRP ${ }^{\mathrm{SPF}}$ neurons was sufficient to induce fear behaviors. Context-dependent optogenetic conditioning was achieved by 10 mins of photo-stimulation in an open field arena; freezing behavior was then assessed in the same context $24 \mathrm{~h}$ after the conditioning (Figure S8M). The ChR2 group exhibited more freezing than the control group, suggesting that CGRP ${ }^{\text {SPFp }}$ activation can act as the US (Figure S8N). For cue-dependent optogenetic conditioning, photo-stimulation was associated with a tone as a non-noxious conditioned stimulus (CS+) in a fear conditioning chamber (Figure S8O). Both context and cue tests were performed after the conditioning. The ChR2 group exhibited more freezing in both context (Figure S8P) and cue tests (Figure S8Q). Together with the optogenetic conditioning results of $\mathrm{CGRP}^{\mathrm{PBel}}$ neurons in the previous result (Han et al., 2015), these results indicate that activation of both CGRP ${ }^{\mathrm{SPF}}$ and $\mathrm{CGRP}^{\mathrm{PBel}}$ neurons is sufficient to induce negative valence associated with affective-motivational pain perception.

We then sought to characterize the functional downstream of the CGRP ${ }^{\mathrm{SPF}}$ neurons. To examine the functional connectivity of anatomical downstream regions from the CGRP ${ }^{\mathrm{SPF}}$ neurons, we performed $e x$ vivo electrophysiology recording. AAV-DIO-ChR2-EYFP was injected into the SPFp of Calca ${ }^{\text {Cre }}$ mice (Figure S9A). After four weeks of ChR2 expression, we performed whole-cell recordings of neurons from AStr, LA, and pIC to measure optogenetically-evoked excitatory/inhibitory postsynaptic currents (EPSC/IPSC; Figure S9B). We found that CGRP ${ }^{\text {SPFp }}$ neurons form functional glutamatergic synapses with neurons within AStr, LA (Figure S9C), and pIC (data not shown). Moreover, the onset of IPSCs lagged 4$5 \mathrm{~ms}$ compared to the onset of EPSCs, indicating a feed-forward inhibition circuit. The number of cells that had both EPSCs and IPSCs, EPSCs only, IPSCs only, and non-responsive were also counted (Figures S9DS9L). To investigate whether these connections form functional circuits that encode negative valence, we optogenetically stimulated axonal terminals from the CGRP ${ }^{\mathrm{SPF}}$ neurons and performed behavioral tests. AAV-DIO-ChR2-EYFP was injected into the SPFp of the Calca ${ }^{C r e}$ mice, and optical fibers were implanted into the postsynaptic areas of CGRP ${ }^{\mathrm{SPFp}}$ neurons, namely the LA, AStr, and pIC (Figure 7D). Optogenetic activation of each of these three projections induced aversion in the RTPA experiment (Figures 7E and S9M), as observed in direct photo-stimulation of CGRP ${ }^{\text {SPFp }}$ cell bodies (Figure 7C). Cue-dependent optogenetic conditioning of the downstream circuits was then performed and only the CGRP ${ }^{\mathrm{SPF} \rightarrow \mathrm{LA}}$ circuit caused significant freezing in the context test (Figure 7F). The other two projections only showed a trend (Figure S9N). Increased freezing was observed in the cue test for all three projections (Figures 7G and S9O), but the most prominent effect was observed with the CGRPSPF $\rightarrow$ LA circuit.

The same behavioral experiments were performed as above with the CGRP ${ }^{\mathrm{PBel}}$ neurons to compare their role in encoding negative valence with the CGRP ${ }^{\mathrm{SPF} p}$ neurons. First, photo-stimulation of the CGRP $\mathrm{PBel}^{\mathrm{PBe}}$ neuronal cell body (Figure 7H) induced aversion during the RTPA test (Figures 7I and 7J). Then, we investigated the CGRP ${ }^{\mathrm{PBel} \rightarrow \mathrm{CeA}}$ circuit as in Figure 7D-7H by optogenetic terminal stimulation (Figure 7K). Photo-stimulation of CGRP ${ }^{\mathrm{PBel} \rightarrow \mathrm{CeA}}$ terminals induced aversion in the RTPA test (Figure 7L), and freezing in the optogenetic conditioning context, and cue tests (Figures $7 \mathrm{M}$ and $7 \mathrm{~N}$ ).

These results satisfy the idea that $\mathrm{CGRP}^{\mathrm{SPFp} \rightarrow \mathrm{LA}}$ and $\mathrm{CGRP}^{\mathrm{PBel} \rightarrow \mathrm{CeA}}$ circuits induce negative valence associated with either affective pain or innate sensory threat cues. 


\section{Discussion}

We report a genetically defined population of neurons that express the neuropeptide CGRP in the SPFp and PBel mediate perception of affective pain and innate sensory threat cues by relaying aversive sensory signals from the spinal cord and other sensory relay areas to the amygdala. These analyses provide the first evidence of convergent innate threat pathways that relay multisensory aversive sensory cues to the amygdala.

\section{CGRP neurons convey affective pain signals to the amygdala}

Perception of pain protects us from physical harm by locating the source of a harmful stimulus. Painful experiences also elicit emotional and motivational responses, which help us remember these events and avoid similar stimuli in the future (Yeh et al., 2018). Thus, pain is not just a simple sensory process but also a complex cognitive process that generates sensory and emotional responses. This unique aspect of pain gives rise to the concept of two aspects of pain: sensory-discriminative and affective-motivational (Auvray et al., 2010; Melzack and Casey, 1968). It is thought that the sensory-discriminative aspect of pain is processed within the sensory cortex via the spino-thalamic tract (STT), and the affective-motivational aspect of pain is processed within the amygdala via the spino-parabrachial tract (SPT). Indeed, previous studies have shown that the PBN-to-CeA circuit is critical for affective-motivational pain perception (Han et al., 2015; Sato et al., 2015). However, it has also been suggested that the thalamus is actively involved in affective-motivational pain perception (Craig, 2003; Willis et al., 2002). Importantly, posterior regions of the thalamus (e.g., the VMpo in primates and humans, as well as the SPFp and PoT in rodents) are anatomically connected to limbic areas (Craig, 1998; Gauriau and Bernard, 2004a) and activated by noxious stimuli (Craig et al., 1994; Peschanski et al., 1981). Therefore, the thalamus likely also plays a critical role in affective-motivational pain perception. Our results demonstrate that a genetically defined population of neurons expressing the neuropeptide CGRP within the SPFp receives monosynaptic inputs from projection neurons within the spinal dorsal horn. They then project to specific nuclei within the amygdala, namely the AStr and LA (Figure 1). Multimodal nociceptive stimuli activate these neurons in an intensity-dependent manner in anesthetized mice (Figure 2). Inactivating these neurons attenuates the perception of affectivemotivational pain, and pain signals induces the changes in synaptic plasticity of these neurons (Figure 3). These data indicate that CGRP ${ }^{\mathrm{SPF}}$ neurons create the spino-thalamo-amygdaloid affective pain pathway.

Unlike the STT, the SPT has been well-characterized as an affective-motivational pain pathway. Recent studies have shown that the lateral PBN receives direct nociceptive inputs from projection neurons within the spinal dorsal horn (Barik et al., 2020; Chiang et al., 2020; Choi et al., 2020; Deng et al., 2020). The dorsolateral PBN (PBdl) predominantly receives nociceptive inputs from the spinal cord and then projects to multiple limbic structures, such as the PAG, VMH, and ILN, thereby producing emotional and physiological changes in response to pain signals (Chiang et al., 2020; Deng et al., 2020). Although the PBdl does not directly project to the amygdala, it indirectly sends pain signals to the CeA through the PBel (Deng et al., 2020). In particular, CGRP ${ }^{\mathrm{PBel}}$ neurons are critical for relaying aversive unconditioned stimuli to the CeA during aversive fear learning (Han et al., 2015). It has been shown that the dynorphin neurons in the PBdl project to the PBel (Chiang et al., 2020), and a recent study has shown that spinal projection neurons that express GPR83 directly innervate CGRP ${ }^{\text {PBel }}$ neurons to relay noxious signals (Choi et al., 2020). Therefore, it is clear that $\mathrm{CGRP}^{\mathrm{PBel}}$ neurons relay nociceptive information from the PBdl and the spinal cord to the CeA. Our retrograde tracing results show that CGRP $^{\text {PBel }}$ neurons receive less direct inputs from the spinal cord than the CGRP ${ }^{\mathrm{SPF} p}$ neurons (Figure 1), and these neurons are activated slower than the CGRP ${ }^{\mathrm{SPFp}}$ 
neurons (Figure S4C and S4J), implying that the CGRP ${ }^{\mathrm{PBel}}$ neurons may receive nociceptive inputs more predominantly through the PBdl than through directly from the spinal cord. The CGRP ${ }^{\text {PBel }}$ neurons also exhibit intensity-dependent activation by multimodal nociceptive stimuli in anesthetized mice (Figure 2) and induce changes in synaptic plasticity by pain signals (Figure 3). Therefore, together with previous studies, our results reaffirm that $\mathrm{CGRP}^{\mathrm{PBel}}$ neurons comprise the spino-parabrachio-amygdaloid pain pathway.

Comparing the CGRP ${ }^{\mathrm{SPF}}$ and $\mathrm{CGRP}^{\mathrm{PBel}}$ neuronal activity in response to multimodal nociceptive stimuli at various intensities in anesthetized mice provides us with novel insights into understanding central affective pain pathways. First, unlike sensory pain signals that are conveyed to the contralateral side of the somatosensory cortex, these neurons are activated by both contralateral and ipsilateral noxious stimuli indicating that the affective pain pathway may not be strictly lateralized (Figure S4). Second, the CGRP ${ }^{\text {SPFp }}$ neurons more robustly respond to the mechanical stimulus, whereas the CGRP ${ }^{\text {PBel }}$ neurons respond more robustly to the thermal stimulus suggesting that these two parallel pathways may convey different modalities of nociceptive information (Figure 2). Lastly, the $\mathrm{CGRP}^{\mathrm{PBel}}$ neurons are activated at lower stimulus intensity but respond slowly to nociceptive signals compared with the CGRP ${ }^{\mathrm{SPF}}$ neurons (Figures 2 and S4). Therefore, our results demonstrate that CGRP-expressing neurons in two brain areas (the SPFp and $\mathrm{PBel}$ ) play complementary roles in relaying multimodal nociceptive signals from the spinal cord to the amygdala through two parallel ascending pain pathways, which are critical for affective-motivational pain perception.

\section{CGRP neurons convey multisensory innate threat cues to the amygdala}

Both pain and innate sensory threats motivate animals to execute immediate avoidance behaviors to escape the threatening or tissue-damaging situation and produce long-lasting aversive memories. Indeed, Pavlovian threat conditioning uses noxious electric foot shock, an acute painful stimulus that motivates the animals to create aversive memory (LeDoux, 2012; Maren, 2001). However, the neural circuit mechanisms by which noxious information is conveyed to the amygdala during aversive learning is not fully understood. Recent advances in the neural circuit-based understanding of innate predator threat perception suggest that innate threat cues from each sensory modality are conveyed to discrete brain areas, such as the amygdala and hypothalamus through parallel pathways (Canteras, 2002; Gross and Canteras, 2012; Kunwar et al., 2015; Silva et al., 2013), which do not overlap with the unconditioned stimulus (affective pain) pathway in Pavlovian threat learning (Silva et al., 2016). However, it is beneficial for animals to use unified neural circuits that encode multimodal aversive sensory signals including pain because animals search for and detect imminent threats using multiple sensory modalities simultaneously. Moreover, previous clinical and animal studies have shown that pain and threat perceptions interact with each other (Berthier et al., 1988; Crook et al., 2014; Elman and Borsook, 2018; Lister et al., 2020). Therefore, it is plausible that there is a unified mechanism that conveys multisensory threat cues from multiple sensory modalities to the amygdala. Our results demonstrate that CGRP-expressing neurons in the PBel and the SPFp not only relay nociceptive stimuli to the amygdala during aversive learning, but also convey innate sensory threat cues from various sensory modalities (Figures 5 and 6). The CGRP ${ }^{S P F p}$ neurons relay aversive sensory cues from the somatosensory, visual, and auditory modalities to the LA, AStr, and pIC. By contrast, CGRP ${ }^{\text {PBel }}$ neurons relay aversive cues from all sensory modalities (somatosensory, visual, auditory, olfactory, and gustatory) (Figures 5, 6, and S3). In addition, previous studies have shown that $\mathrm{CGRP}^{\mathrm{PBel}}$ neurons are activated by hypercapnic conditions (high $\mathrm{CO}_{2}$ levels) (Kaur et al., 2017; Yokota et al., 2015), and aversive visceral 
cues, such as lithium chloride and lipopolysaccharide (Carter et al., 2013; Paues et al., 2001). Therefore, it is tempting to speculate that $\mathrm{CGRP}^{\mathrm{SPFp}}$ neurons relay exteroceptive threat signals, whereas $\mathrm{CGRP}^{\mathrm{PBel}}$ neurons relay both exteroceptive and interoceptive threat cues to the amygdala. Consistent with a previous report (Han et al., 2015), our data provide strong evidence that the CGRP ${ }^{\text {PBel }}$ neurons mediate aversive learning via their projection to the CeA (Figure $7 \mathrm{~K}-7 \mathrm{~N}$ ). Our results seem to be contradictory to a recent study by Bowen et al., (2020), which argued that optogenetic stimulation of $\mathrm{CGRP}^{\mathrm{PBel}}$ axon terminals in the VPMpc, instead of the CeA, evokes strong aversive memory. This discrepancy can be explained by the differences in optogenetic stimulation protocols. Whereas we used a $40 \mathrm{~Hz}$ light stimulation for $10 \mathrm{~s}$ as described (Han et al., 2015), Bowen et al. used a $30 \mathrm{~Hz}$ light train for only $2 \mathrm{~s}$. Moreover, it is worth noting that axonal bundles from all glutamatergic neurons in the PBel that project to forebrain regions, including the CeA, BNST, and PSTN pass through the VPMpc (Huang et al., 2020), which suggests that optogenetic stimulation within the VPMpc activates both CGRP ${ }^{\mathrm{PBel}}$ axonal terminals and axon bundles passing through this area. Therefore, an alternative explanation of their observation is that concurrent stimulation of all downstream areas produces stronger aversive memory than stimulating CeA alone.

Together, our results demonstrate that the US pathways for relaying electric foot shock cues during the Pavlovian fear learning also collectively relay innate threat cues from multiple sensory modalities.

\section{Pain-threat interactions in affective pain disorders}

Our results show that the affective-motivational pain and multisensory threat stimuli arrive in the amygdala via the same neural pathways. Interestingly, pain-threat interactions have been reported in many human clinical cases. People with pain asymbolia, caused by damage to limbic brain areas, have the normal sensory perception of noxious stimuli, but they have impaired affective pain perception (Berthier et al., 1988; Rubins and Friedman, 1948). Interestingly, pain asymbolia patients often display deficits in perceiving general threats (Klein, 2015; Price, 2000), indicating that the perception of affective pain and other sensory threat cues share the same neural substrate. People with congenital insensitivity to pain (CIP) are insensitive to all sensory and affective components of pain, but they also display profound deficits in general threat perception, which is the primary cause of their short life expectancy (McMurray, 1955; Nagasako et al., 2003). CIP is caused by loss-of-function mutations in genes critical for pain transmission, such as $\operatorname{Scn} 9 a$ (Dabby, 2012; Fischer and Waxman, 2010; Lampert et al., 2010) and Faah (Drissi et al., 2020). Scn9a encodes voltage-gated sodium channel type 7 ( $N a v 1.7)$, and Faah encodes fatty acid amide hydrolase, both of which are critical for pain transmission in the spinal cord (Cajanus et al., 2016; Kim et al., 2006; Nantermet and Henze, 2011; Nassar et al., 2004). However, the functional loss of these genes in the spinal neurons cannot explain the insensitivity to general threats exhibited by CIP patients. Surprisingly, our cell type-specific transcriptome analysis revealed that $S c n 9 a$ and Faah transcripts are highly enriched in both CGRP $^{\text {PBel }}$ and CGRP ${ }^{\mathrm{SPF}}$ neurons (Figure 4). Thus, mutations in these genes may prevent these neurons from relaying sensory threat signals to the amygdala, thereby causing insensitivity to general threats in CIP. This speculation should be addressed by testing the causal relationship between mutations in these genes in CGRP neurons and threat perception.

Opposite clinical cases also exist. People with affective pain disorders, such as migraine, and fibromyalgia experience chronic pain and suffer from hypersensitivity to normal sensory stimuli (Demarquay and 
Mauguière, 2016; Harriott and Schwedt, 2014; Harte et al., 2016; López-Solà et al., 2017). Further, normal sensory stimuli often trigger or aggravate their pain symptoms (Bar-Shalita and Cermak, 2020; Bar-Shalita et al., 2019). Surprisingly, the gene Cacnala, which has been linked to migraines, is highly enriched in both CGRP ${ }^{\mathrm{PBel}}$ and CGRP ${ }^{\mathrm{SPF}}$ neurons (Figure 4). Further studies should address the causal relationship 523 between loss of Cacnala function in CGRP neurons and sensory hypersensitivity in migraine. In addition, 524 CGRP signaling is a proven therapeutic target for treating migraine (Ashina, 2020). Therefore, we speculate that the CGRP-expressing neurons characterized in this study may serve as the functional substrate for 526 sensory hypersensitivity in migraine. Identifying membrane proteins commonly expressed in both the $527 \mathrm{CGRP}^{\mathrm{PBel}}$ and CGRP ${ }^{\mathrm{SPFp}}$ neurons (Figure 4C) may provide potential candidates of therapeutic targets for 528 treating affective pain-, and innate threat-related disorders, such as migraine, fibromyalgia, phobias, panic 529 disorder, and post-traumatic stress disorder. Indeed, the CGRP receptor antagonists or neutralizing monoclonal antibodies are promising drugs for treating migraine, but they may also serve as potential therapeutic interventions for treating the disorders mentioned above.

\section{Conclusion}

Our findings demonstrate that the CGRP ${ }^{\text {SPFp }}$ neurons create a novel spino-thalamo-amygdaloid affective pain pathway and, together with the previously characterized CGRP ${ }^{\mathrm{PBel}}$ neurons, serve as complementary parallel pathways for conveying the unconditioned stimulus during Pavlovian threat learning, as well as multisensory innate threat cues from all sensory modalities to the amygdala (Figure S10). The discovery of a unified threat perception system that conveys multimodal interoceptive and exteroceptive aversive sensory stimuli greatly enhances our understanding of the neural mechanisms of innate threat perception. These insights also provide opportunities to discover novel targets for developing therapeutic interventions against affective pain- and innate threat-related disorders. 


\section{Acknowledgments}

544 We thank Dr. D. O'Keefe, Ms. C. Jia, and Han lab members for critical discussions during manuscript 545 preparation. S.H. is supported by 1R01MH116203 from NIMH and the Bridge to Independence award from 546 the Simons Foundation Autism Research Initiative (SFARI \#388708). S.L. is supported by the Salk Women $547 \&$ Science Special Award, the Mary K. Chapman Foundation, and the Jesse \& Caryl Philips Foundation.

\section{Author Contributions}

552 S.H. conceived of the idea and secured funding. S.H., S.J.K., S.L., and M.Y. designed and performed the 553 experiments. S.H. and S.J.K wrote the manuscript. S.J.K. performed most of the CGRP ${ }^{\text {SPFp }}$ experiments. 554 S.L., and S.J.K. performed CGRP ${ }^{\text {PBel }}$ experiments. M.Y. performed electrophysiology and CGRP ${ }^{\text {PBel }}$ RTPA. 555 D.I.K., and J.H.K. performed RiboTag experiments, and T.G.O analyzed it. R.M.E. provided resources for 556 Ribotag analysis. J.P. performed spinal projection histology experiments. M.G. provided $C d x 2^{F l p O}$ mouse 557 line. K.F.L. provided resources for microscopy.

558

560 The authors declare no competing interests. 


\section{References}

Ashina, M. (2020). Migraine. N. Engl. J. Med. 383, 1866-1876.

Auvray, M., Myin, E., and Spence, C. (2010). The sensory-discriminative and affectivemotivational aspects of pain. Neurosci. Biobehav. Rev. 34, 214-223.

Bach, D.R., Hurlemann, R., and Dolan, R.J. (2015). Impaired threat prioritisation after selective bilateral amygdala lesions. Cortex 63, 206-213.

Barik, A., Sathyamurthy, A., Thompson, J., Seltzer, M., Levine, A., and Chesler, A. (2020). A spinoparabrachial circuit defined by Tacr1 expression drives pain. BioRxiv 2020.07.15.205484.

Bar-Shalita, T., and Cermak, S.A. (2020). Multi-sensory Responsiveness and Personality Traits Predict Daily Pain Sensitivity. Front. Integr. Neurosci. 13, 77.

Bar-Shalita, T., Granovsky, Y., Parush, S., and Weissman-Fogel, I. (2019). Sensory Modulation Disorder (SMD) and Pain: A New Perspective. Front. Integr. Neurosci. 13.

Barsy, B., Kocsis, K., Magyar, A., Babiczky, Á., Szabó, M., Veres, J.M., Hillier, D., Ulbert, I., Yizhar, O., and Mátyás, F. (2020). Associative and plastic thalamic signaling to the lateral amygdala controls fear behavior. Nat. Neurosci. 23, 625-637.

Basbaum, A.I., Bautista, D.M., Scherrer, G., and Julius, D. (2009). Cellular and Molecular Mechanisms of Pain. Cell 139, 267-284.

Bernard, J.F., Huang, G.F., and Besson, J.M. (1992). Nucleus centralis of the amygdala and the globus pallidus ventralis: electrophysiological evidence for an involvement in pain processes. J. Neurophysiol. 68, 551-569.

Berthier, M., Starkstein, S., and Leiguarda, R. (1988). Asymbolia for pain: A sensory-limbic disconnection syndrome. Ann. Neurol. 24, 41-49.

Blanchard, D.C., and Blanchard, R.J. (1972). Innate and conditioned reactions to threat in rats with amygdaloid lesions. J. Comp. Physiol. Psychol. 81, 281-290.

Bourane, S., Grossmann, K.S., Britz, O., Dalet, A., Del Barrio, M.G., Stam, F.J., GarciaCampmany, L., Koch, S., and Goulding, M. (2015). Identification of a Spinal Circuit for Light Touch and Fine Motor Control. Cell 160, 503-515.

Bowen, A.J., Chen, J.Y., Huang, Y.W., Baertsch, N.A., Park, S., and Palmiter, R.D. (2020). Dissociable control of unconditioned responses and associative fear learning by parabrachial CGRP neurons. ELife 9, e59799.

Bushnell, M.C., Čeko, M., and Low, L.A. (2013). Cognitive and emotional control of pain and its disruption in chronic pain. Nat. Rev. Neurosci. 14, 502-511. 
Cajanus, K., Holmström, E.J., Wessman, M., Anttila, V., Kaunisto, M.A., and Kalso, E. (2016). Effect of endocannabinoid degradation on pain: role of: FAAH: polymorphisms in experimental and postoperative pain in women treated for breast cancer. PAIN 157, 361-369.

Canteras, N.S. (2002). The medial hypothalamic defensive system: Hodological organization and functional implications. Pharmacol. Biochem. Behav. 71, 481-491.

Carter, M.E., Soden, M.E., Zweifel, L.S., and Palmiter, R.D. (2013). Genetic identification of a neural circuit that suppresses appetite. Nature advance online publication.

Chen, J.Y., Campos, C.A., Jarvie, B.C., and Palmiter, R.D. (2018). Parabrachial CGRP Neurons Establish and Sustain Aversive Taste Memories. Neuron 100, 891-899.e5.

Chiang, M.C., Nguyen, E.K., Canto-Bustos, M., Papale, A.E., Oswald, A.-M.M., and Ross, S.E. (2020). Divergent Neural Pathways Emanating from the Lateral Parabrachial Nucleus Mediate Distinct Components of the Pain Response. Neuron 106, 927-939.e5.

Choi, S., Hachisuka, J., and Ginty, D. (2020). Parallel ascending spinal pathways for affective touch and pain. Nature In press.

Coolen, L.M., Veening, J.G., Wells, A.B., and Shipley, M.T. (2003a). Afferent connections of the parvocellular subparafascicular thalamic nucleus in the rat: Evidence for functional subdivisions. J. Comp. Neurol. 463, 132-156.

Coolen, L.M., Veening, J.G., Petersen, D.W., and Shipley, M.T. (2003b). Parvocellular subparafascicular thalamic nucleus in the rat: Anatomical and functional compartmentalization. J. Comp. Neurol. 463, 117-131.

Craig, A.D. (1998). A new version of the thalamic disinhibition hypothesis of central pain. Pain Forum 7, 1-14.

Craig, A.D. (Bud) (2003). PAIN MECHANISMS: Labeled Lines Versus Convergence in Central Processing. Annu. Rev. Neurosci. 26, 1-30.

Craig, A.D., Bushnell, M.C., Zhang, E.-T., and Blomqvist, A. (1994). A thalamic nucleus specific for pain and temperature sensation. Nature 372, 770-773.

Craig, A.D., Chen, K., Bandy, D., and Reiman, E.M. (2000). Thermosensory activation of insular cortex. Nat. Neurosci. 3, 184-190.

Cravatt, B.F., and Lichtman, A.H. (2004). The endogenous cannabinoid system and its role in nociceptive behavior. J. Neurobiol. 61, 149-160.

Crook, R.J., Dickson, K., Hanlon, R.T., and Walters, E.T. (2014). Nociceptive Sensitization Reduces Predation Risk. Curr. Biol. 24, 1121-1125.

Dabby, R. (2012). Pain Disorders and Erythromelalgia Caused by Voltage-Gated Sodium Channel Mutations. Curr. Neurol. Neurosci. Rep. 12, 76-83. 
Dado, R.J., Katter, J.T., and Giesler, G.J. (1994). Spinothalamic and spinohypothalamic tract neurons in the cervical enlargement of rats. II. Responses to innocuous and noxious mechanical and thermal stimuli. J. Neurophysiol. 71, 981-1002.

Dal Monte, O., Costa, V.D., Noble, P.L., Murray, E.A., and Averbeck, B.B. (2015). Amygdala lesions in rhesus macaques decrease attention to threat. Nat. Commun. 6, 10161.

Demarquay, G., and Mauguière, F. (2016). Central Nervous System Underpinnings of Sensory Hypersensitivity in Migraine: Insights from Neuroimaging and Electrophysiological Studies. Headache J. Head Face Pain 56, 1418-1438.

Deng, J., Zhou, H., Lin, J.-K., Shen, Z.-X., Chen, W.-Z., Wang, L.-H., Li, Q., Mu, D., Wei, Y.-C., $\mathrm{Xu}, \mathrm{X} .-\mathrm{H}$. , et al. (2020). The Parabrachial Nucleus Directly Channels Spinal Nociceptive Signals to the Intralaminar Thalamic Nuclei, but Not the Amygdala. Neuron 107, 923.

D'Hanis, W., Linke, R., and Yilmazer-Hanke, D. m. (2007). Topography of thalamic and parabrachial calcitonin gene-related peptide (CGRP) immunoreactive neurons projecting to subnuclei of the amygdala and extended amygdala. J. Comp. Neurol. 505, 268-291.

Dobolyi, A., Irwin, S., Makara, G., Usdin, T.B., and Palkovits, M. (2005). Calcitonin gene-related peptide-containing pathways in the rat forebrain. J. Comp. Neurol. 489, 92-119.

Drissi, I., Woods, W.A., and Woods, C.G. (2020). Understanding the genetic basis of congenital insensitivity to pain. Br. Med. Bull. 133, 65-78.

Elman, I., and Borsook, D. (2018). Threat Response System: Parallel Brain Processes in Pain visà-vis Fear and Anxiety. Front. Psychiatry 9.

Fischer, T.Z., and Waxman, S.G. (2010). Familial pain syndromes from mutations of the Nav1.7 sodium channel. Ann. N. Y. Acad. Sci. 1184, 196-207.

Gao, Y.-J., Ren, W.-H., Zhang, Y.-Q., and Zhao, Z.-Q. (2004). Contributions of the anterior cingulate cortex and amygdala to pain- and fear-conditioned place avoidance in rats: Pain 110, 343-353.

Gauriau, C., and Bernard, J.-F. (2002). Pain pathways and parabrachial circuits in the rat. Exp. Physiol. 87, 251-258.

Gauriau, C., and Bernard, J.-F. (2004a). Posterior Triangular Thalamic Neurons Convey Nociceptive Messages to the Secondary Somatosensory and Insular Cortices in the Rat. J. Neurosci. $24,752-761$.

Gauriau, C., and Bernard, J.-F. (2004b). A comparative reappraisal of projections from the superficial laminae of the dorsal horn in the rat: The forebrain. J. Comp. Neurol. 468, 24-56.

Gross, C.T., and Canteras, N.S. (2012). The many paths to fear. Nat. Rev. Neurosci. 13, 651-658. 
Han, S., Soleiman, M.T., Soden, M.E., Zweifel, L.S., and Palmiter, R.D. (2015). Elucidating an Affective Pain Circuit that Creates a Threat Memory. Cell 162, 363-374.

Harriott, A.M., and Schwedt, T.J. (2014). Migraine is Associated With Altered Processing of Sensory Stimuli. Curr. Pain Headache Rep. 18, 458.

Harte, S.E., Ichesco, E., Hampson, J.P., Peltier, S.J., Schmidt-Wilcke, T., Clauw, D.J., and Harris, R.E. (2016). Pharmacologic attenuation of cross-modal sensory augmentation within the chronic pain insula: PAIN 157, 1933-1945.

Helmstetter, F.J. (1992). The Amygdala Is Essential for the Expression of Conditional Hypoalgesia. Behav. Neurosci. 106, 518-528.

Huang, D., Grady, F.S., Peltekian, L., and Geerling, J.C. (2020). Efferent projections of VGLUT2, FOXP2, and PDYN parabrachial neurons in mice. J. Comp. Neurol. cne.24975.

Janak, P.H., and Tye, K.M. (2015). From circuits to behaviour in the amygdala. Nature 517, 284292.

Julius, D., and Basbaum, A.I. (2001). Molecular mechanisms of nociception. Nature 413, 203210.

Kaur, S., Wang, J.L., Ferrari, L., Thankachan, S., Kroeger, D., Venner, A., Lazarus, M., Wellman, A., Arrigoni, E., Fuller, P.M., et al. (2017). A Genetically Defined Circuit for Arousal from Sleep during Hypercapnia. Neuron 96, 1153-1167.e5.

Kim, E.J., Jacobs, M.W., Ito-Cole, T., and Callaway, E.M. (2016). Improved Monosynaptic Neural Circuit Tracing Using Engineered Rabies Virus Glycoproteins. Cell Rep. 15, 692-699.

Kim, H., Mittal, D.P., Iadarola, M.J., and Dionne, R.A. (2006). Genetic predictors for acute experimental cold and heat pain sensitivity in humans. J. Med. Genet. 43, e40-e40.

Kim, J., Zhang, X., Muralidhar, S., LeBlanc, S.A., and Tonegawa, S. (2017). Basolateral to Central Amygdala Neural Circuits for Appetitive Behaviors. Neuron 93, 1464-1479.e5.

Klein, C. (2015). What Pain Asymbolia Really Shows. Mind 124, 493-516.

Kunwar, P.S., Zelikowsky, M., Remedios, R., Cai, H., Yilmaz, M., Meister, M., and Anderson, D.J. (2015). Ventromedial hypothalamic neurons control a defensive emotion state. ELife 4, e06633.

Lampert, A., O'Reilly, A.O., Reeh, P., and Leffler, A. (2010). Sodium channelopathies and pain. Pflüg. Arch. - Eur. J. Physiol. 460, 249-263.

LeDoux, J. (2007). The amygdala. Curr. Biol. 17, R868-R874.

LeDoux, J. (2012). Rethinking the Emotional Brain. Neuron 73, 653-676.

LeDoux, J.E. (2000). Emotion Circuits in the Brain. Annu. Rev. Neurosci. 23, 155-184. 
LeDoux, J.E., Ruggiero, D.A., and Reis, D.J. (1985). Projections to the subcortical forebrain from anatomically defined regions of the medial geniculate body in the rat. J. Comp. Neurol. 242, 182213.

Lister, K.C., Bouchard, S.M., Markova, T., Aternali, A., Denecli, P., Pimentel, S.D., Majeed, M., Austin, J.-S., de C. Williams, A.C., and Mogil, J.S. (2020). Chronic pain produces hypervigilance to predator odor in mice. Curr. Biol. 30, R866-R867.

López-Solà, M., Woo, C.-W., Pujol, J., Deus, J., Harrison, B.J., Monfort, J., and Wager, T.D. (2017). Towards a neurophysiological signature for fibromyalgia. PAIN 158, 34-47.

Manning, B.H., and Mayer, D.J. (1995). The central nucleus of the amygdala contributes to the production of morphine antinociception in the formalin test. PAIN® 63, 141-152.

Maren, S. (2001). Neurobiology of Pavlovian Fear Conditioning. Annu. Rev. Neurosci. 24, 897931.

McMurray, G.A. (1955). Congenital insensitivity to pain and its implications for motivational theory. Can. J. Psychol. Can. Psychol. 9, 121-131.

Melzack, R., and Casey, K. (1968). Sensory, Motivational, and Central Control Determinants of Pain. In Skin Senses, pp. 423-439.

Nagasako, E.M., Oaklander, A.L., and Dworkin, R.H. (2003). Congenital insensitivity to pain: an update. PAIN 101, 213-219.

Nantermet, P.G., and Henze, D.A. (2011). Recent Advances Toward Pain Therapeutics. In Annual Reports in Medicinal Chemistry, (Elsevier), pp. 19-32.

Nassar, M.A., Stirling, L.C., Forlani, G., Baker, M.D., Matthews, E.A., Dickenson, A.H., and Wood, J.N. (2004). Nociceptor-specific gene deletion reveals a major role for Nav1.7 (PN1) in acute and inflammatory pain. Proc. Natl. Acad. Sci. 101, 12706-12711.

Neugebauer, V. (2015). Amygdala Pain Mechanisms. In Pain Control, (Springer, Berlin, Heidelberg), pp. 261-284.

Palmiter, R.D. (2018). The Parabrachial Nucleus: CGRP Neurons Function as a General Alarm. Trends Neurosci. 41, 280-293.

Paues, J., Engblom, D., Mackerlova, L., Ericsson-Dahlstrand, A., and Blomqvist, A. (2001). Feeding-related immune responsive brain stem neurons: association with CGRP. NeuroReport 12, 2399-2403.

Peschanski, M., Guilbaud, G., and Gautron, M. (1981). Posterior intralaminar region in rat: Neuronal responses to noxious and nonnoxious cutaneous stimuli. Exp. Neurol. 72, 226-238.

Price, D.D. (1999). Multisensory integration in pain and consciousness. Pain Forum 8, 130-132. 
Price, D.D. (2000). Psychological and Neural Mechanisms of the Affective Dimension of Pain. Science 288, 1769-1772.

Price, D.D. (2002). Central Neural Mechanisms that Interrelate Sensory and Affective Dimensions of Pain. Mol. Interv. 2, 392.

Ren, W., and Neugebauer, V. (2010). Pain-related increase of excitatory transmission and decrease of inhibitory transmission in the central nucleus of the amygdala are mediated by mGluR1. Mol. Pain 6, 93.

Ressler, R.L., and Maren, S. (2019). Synaptic encoding of fear memories in the amygdala. Curr. Opin. Neurobiol. 54, 54-59.

Rosen, J.B., Asok, A., and Chakraborty, T. (2015). The smell of fear: innate threat of 2,5-dihydro2,4,5-trimethylthiazoline, a single molecule component of a predator odor. Front. Neurosci. 9.

Rubins, J.L., and Friedman, E.D. (1948). ASYMBOLIA FOR PAIN. Arch. Neurol. Psychiatry 60, 554-573.

Russell, F.A., King, R., Smillie, S.-J., Kodji, X., and Brain, S.D. (2014). Calcitonin Gene-Related Peptide: Physiology and Pathophysiology. Physiol. Rev. 94, 1099-1142.

Russo, A.F. (2015). Calcitonin Gene-Related Peptide (CGRP): A New Target for Migraine. Annu. Rev. Pharmacol. Toxicol. 55, 533-552.

Salay, L.D., Ishiko, N., and Huberman, A.D. (2018). A midline thalamic circuit determines reactions to visual threat. Nature 557, 183.

Sanz, E., Yang, L., Su, T., Morris, D.R., McKnight, G.S., and Amieux, P.S. (2009). Cell-typespecific isolation of ribosome-associated mRNA from complex tissues. Proc. Natl. Acad. Sci. 106, 13939-13944.

Sato, M., Ito, M., Nagase, M., Sugimura, Y.K., Takahashi, Y., Watabe, A.M., and Kato, F. (2015). The lateral parabrachial nucleus is actively involved in the acquisition of fear memory in mice. Mol. Brain 8, 22.

Shi, C., and Davis, M. (1999). Pain Pathways Involved in Fear Conditioning Measured with FearPotentiated Startle: Lesion Studies. J. Neurosci. 19, 420-430.

Shinohara, K., Watabe, A.M., Nagase, M., Okutsu, Y., Takahashi, Y., Kurihara, H., and Kato, F. (2017). Essential role of endogenous calcitonin gene-related peptide in pain-associated plasticity in the central amygdala. Eur. J. Neurosci. 46, 2149-2160.

Silva, B.A., Mattucci, C., Krzywkowski, P., Murana, E., Illarionova, A., Grinevich, V., Canteras, N.S., Ragozzino, D., and Gross, C.T. (2013). Independent hypothalamic circuits for social and predator fear. Nat. Neurosci. 16, 1731-1733. 
Silva, B.A., Gross, C.T., and Gräff, J. (2016). The neural circuits of innate fear: detection, integration, action, and memorization. Learn. Mem. 23, 544-555.

Simons, L.E., Moulton, E.A., Linnman, C., Carpino, E., Becerra, L., and Borsook, D. (2014). The human amygdala and pain: Evidence from neuroimaging. Hum. Brain Mapp. 35, 527-538.

Stamatakis, A.M., and Stuber, G.D. (2012). Activation of lateral habenula inputs to the ventral midbrain promotes behavioral avoidance. Nat. Neurosci. 15, 1105-1107.

Tanimoto, S., Nakagawa, T., Yamauchi, Y., Minami, M., and Satoh, M. (2003). Differential contributions of the basolateral and central nuclei of the amygdala in the negative affective component of chemical somatic and visceral pains in rats. Eur. J. Neurosci. 18, 2343-2350.

Thompson, J.M., and Neugebauer, V. (2017). Amygdala Plasticity and Pain. Pain Res. Manag. 2017.

Todd, A.J. (2010). Neuronal circuitry for pain processing in the dorsal horn. Nat. Rev. Neurosci. $11,823-836$.

Tong, W.H., Abdulai-Saiku, S., and Vyas, A. (2020). Medial Amygdala Arginine Vasopressin Neurons Regulate Innate Aversion to Cat Odors in Male Mice. Neuroendocrinology.

Veinante, P., Yalcin, I., and Barrot, M. (2013). The amygdala between sensation and affect: a role in pain. J. Mol. Psychiatry 1.

Wang, L., Gillis-Smith, S., Peng, Y., Zhang, J., Chen, X., Salzman, C.D., Ryba, N.J.P., and Zuker, C.S. (2018). The coding of valence and identity in the mammalian taste system. Nature 558, 127131.

Wei, P., Liu, N., Zhang, Z., Liu, X., Tang, Y., He, X., Wu, B., Zhou, Z., Liu, Y., Li, J., et al. (2015). Processing of visually evoked innate fear by a non-canonical thalamic pathway. Nat. Commun. 6 , 6756.

Willis, W.D., Zhang, X., Honda, C.N., and Giesler, G.J. (2002). A critical review of the role of the proposed VMpo nucleus in pain. J. Pain 3, 79-94.

Xiu, J., Zhang, Q., Zhou, T., Zhou, T., Chen, Y., and Hu, H. (2014). Visualizing an emotional valence map in the limbic forebrain by TAI-FISH. Nat. Neurosci. 17, 1552-1559.

Yasui, Y., Saper, C.B., and Cechetto, D.F. (1991). Calcitonin gene-related peptide (CGRP) immunoreactive projections from the thalamus to the striatum and amygdala in the rat. J. Comp. Neurol. 308, 293-310.

Yeh, L.-F., Watanabe, M., Sulkes-Cuevas, J., and Johansen, J.P. (2018). Dysregulation of aversive signaling pathways: a novel circuit endophenotype for pain and anxiety disorders. Curr. Opin. Neurobiol. 48, 37-44. 
793 Yokota, S., Kaur, S., VanderHorst, V.G., Saper, C.B., and Chamberlin, N.L. (2015). Respiratory794 related outputs of glutamatergic, hypercapnia-responsive parabrachial neurons in mice. J. Comp.

795 Neurol. 523, 907-920.

796 Yu, L.-C., Hou, J.-F., Fu, F.-H., and Zhang, Y.-X. (2009). Roles of calcitonin gene-related peptide 797 and its receptors in pain-related behavioral responses in the central nervous system. Neurosci. 798 Biobehav. Rev. 33, 1185-1191.

799 Zhang, X., and Giesler, G.J. (2005). Response Characterstics of Spinothalamic Tract Neurons That 800 Project to the Posterior Thalamus in Rats. J. Neurophysiol. 93, 2552-2564.

801 Zhou, Z., Liu, X., Chen, S., Zhang, Z., Liu, Y., Montardy, Q., Tang, Y., Wei, P., Liu, N., Li, L., et 802 al. (2019). A VTA GABAergic Neural Circuit Mediates Visually Evoked Innate Defensive 803 Responses. Neuron 103, 473-488.e6. 
Figure Legends

A

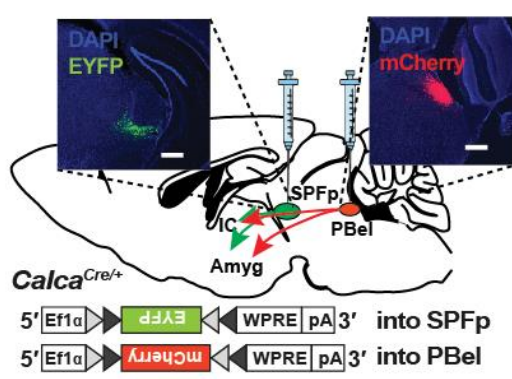

C

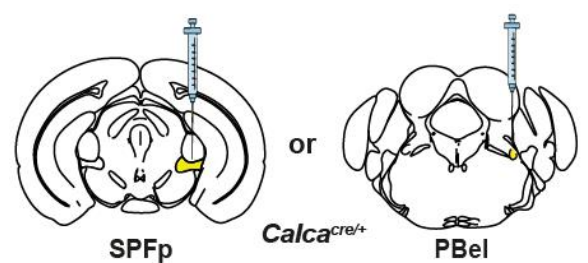

B

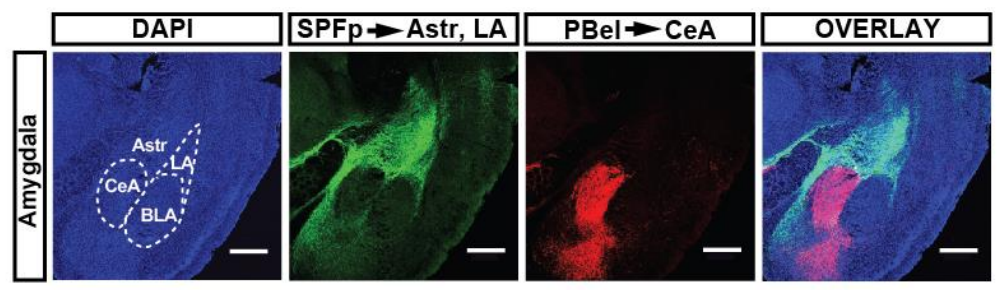

$5^{\prime}$ Syn $D N=+\forall M \perp$ WPRE $\mathrm{pA} 3^{\prime}$ $\downarrow$ Calca $^{\text {cre/+ }}$ 5 'Syn $>$ TVA + G WPRE $P A$ 3' $\downarrow$ 2-3 weeks EnvA RVdG-mCherry

\section{D}
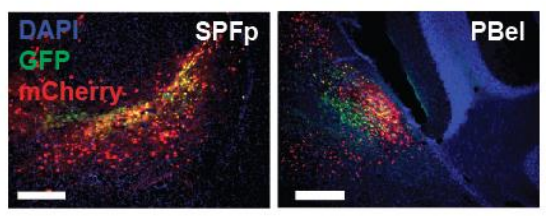

E

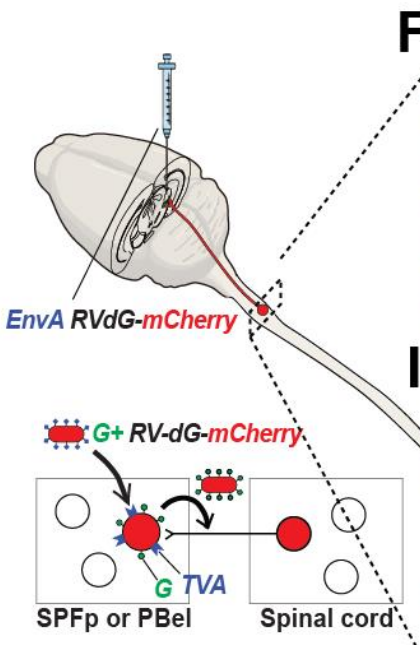

806

807

808

809

810

811

812

813

814

815

816

817

818

819

820

821
F

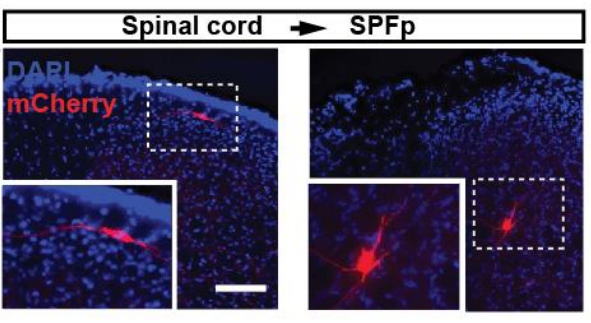

G
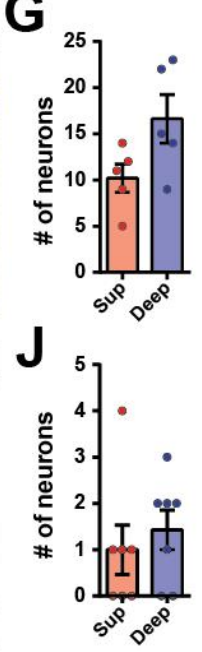

H

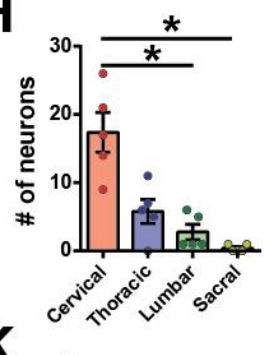

K

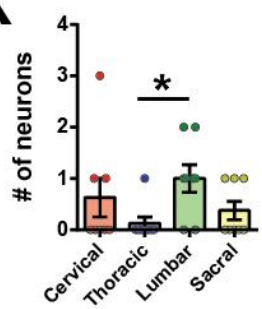

Figure 1. CGRP ${ }^{\mathrm{SPF}}$ and CGRP ${ }^{\mathrm{PBel}}$ neurons form spino-thalamo-amygdaloid and spino-parabrachioamygdaloid pathways.

(A) Schematic and representative images of Cre-dependent expression of EYFP in the SPFp and mCherry in the PBel of a Calca ${ }^{\text {Cre }}$ mouse. Scale bars, $200 \mu \mathrm{m}$.

(B) The axonal projections from the CGRP ${ }^{\mathrm{SPFp}}$ and CGRP ${ }^{\mathrm{PBel}}$ neurons are mutually exclusive in the amygdala sub-regions. Scale bars, $500 \mu \mathrm{m}$.

(C) Schematic diagrams and images of Cre-dependent expression of TVA and G in SPFp or PBel neurons of $\mathrm{Calca}^{\mathrm{Cre}}$ mice for the rabies tracing.

(D) Representative images of the SPFp and PBel five days after EnvA-RVdG-mCherry injection. Yellow indicates the starter cells. Scale bars, $200 \mu \mathrm{m}$.

(E) A schematic diagram of identifying presynaptic neurons by monosynaptic rabies tracing.

(F) Representative images of superficial and deep layer dorsal horn neurons that project to CGRP ${ }^{\text {SPFp }}$ neurons.

(G) The number of spinal dorsal horn neurons in the superficial (Sup) and deep (deep) layers project to the CGRP ${ }^{\text {SPFp }}$ neurons. 
822 (H) The number of spinal cord neurons in different spinal segments that project to the CGRP ${ }^{\text {SPFp }}$ neurons.

823 (I) Representative images of the superficial dorsal horn and lateral spinal nucleus neurons project to 824 CGRP $^{\text {PBel }}$ neurons.

825 (J) The number of spinal dorsal horn neurons in the superficial (Sup) and deep (deep) layers project to the 826 CGRP $^{\mathrm{PBel}}$ neurons.

827 (K) The number of spinal cord neurons in different spinal segments that project to the CGRP ${ }^{\text {PBel }}$ neurons.

828

829

830

831

\section{Statistics}

(G) Superficial: $10.20 \pm 1.53$, Deep: $16.60 \pm 2.62(\mathrm{n}=5)$. Paired t-test (two-tailed), $\mathrm{p}=0.0723$. (H) Cervical: $17.40 \pm 2.91$, Thoracic: 5.80 \pm 2.91 , Lumbar: $2.80 \pm 1.11$, Sacral: $0.40 \pm 0.24(n=5)$. Repeated measure one-way ANOVA, $\mathrm{p}=0.0017$. Cervical group was significantly different with lumbar $(\mathrm{p}<0.05)$ and sacral $(\mathrm{p}<0.05$, Tukey's multiple comparisons). (J) Superficial: $1.00 \pm 0.53$, Deep: $1.43 \pm 0.43(\mathrm{n}=8)$. Paired t-test (two-tailed), $\mathrm{p}=0.6286$. (K) Cervical: $0.63 \pm 0.38$, Thoracic: $0.13 \pm 0.13$, Lumbar: $1.00 \pm 0.27$, Sacral: $0.38 \pm 0.18(n=8)$. Repeated measure one-way ANOVA, $\mathrm{p}=0.1615$. Thoracic group was significantly different with lumbar $(\mathrm{p}<0.05$, Tukey's multiple comparisons). 

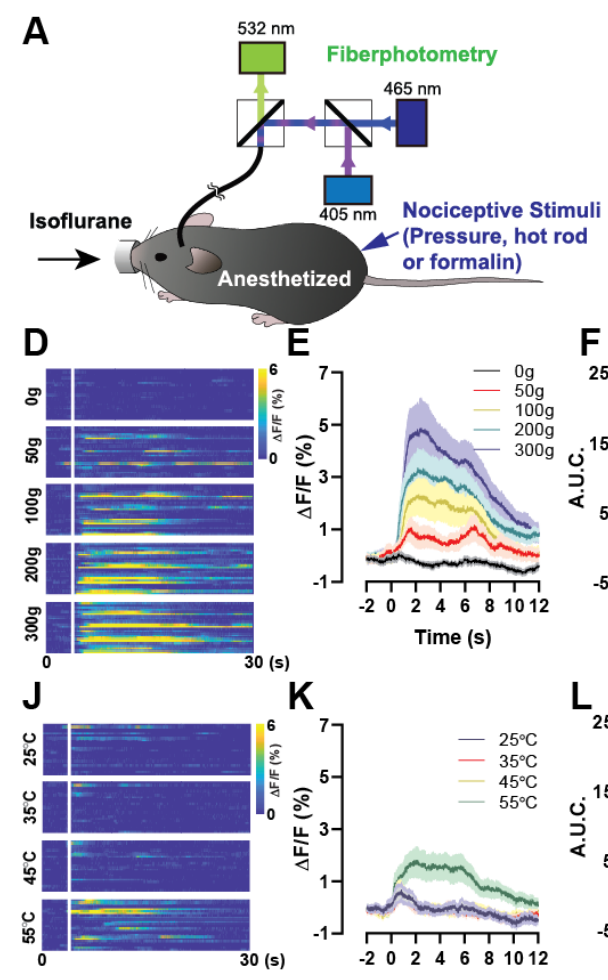

$E_{7}$
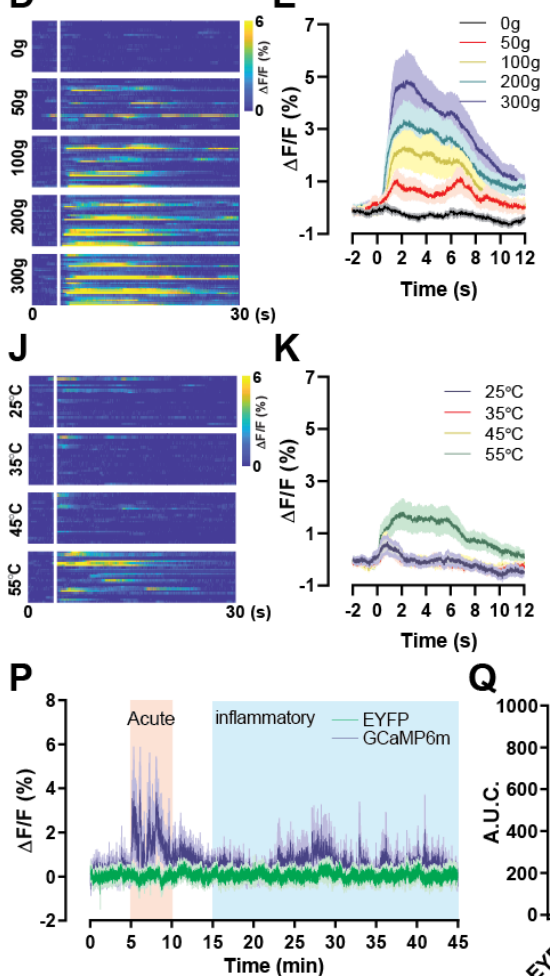

840

841

842

843

844

845

846

847

848

849

850

851

852

853

854

855

856 above the SPFp and PBel. Scale bars, $200 \mu \mathrm{m}$. $(0,50,100,200$, and $300 \mathrm{~g})$. $\operatorname{rod}\left(25,35,45\right.$, or $\left.55^{\circ} \mathrm{C}\right)$. controlled rod. the paw. $10 \mathrm{~min}$ ) of formalin response.

Figure 2. CGRP ${ }^{\text {SPFp }}$ and CGRP ${ }^{\text {PBel }}$ neurons are activated by multimodal nociceptive stimuli.
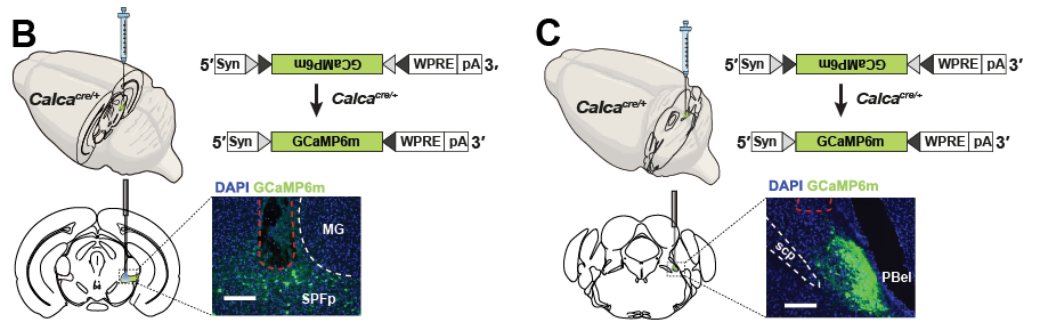

G
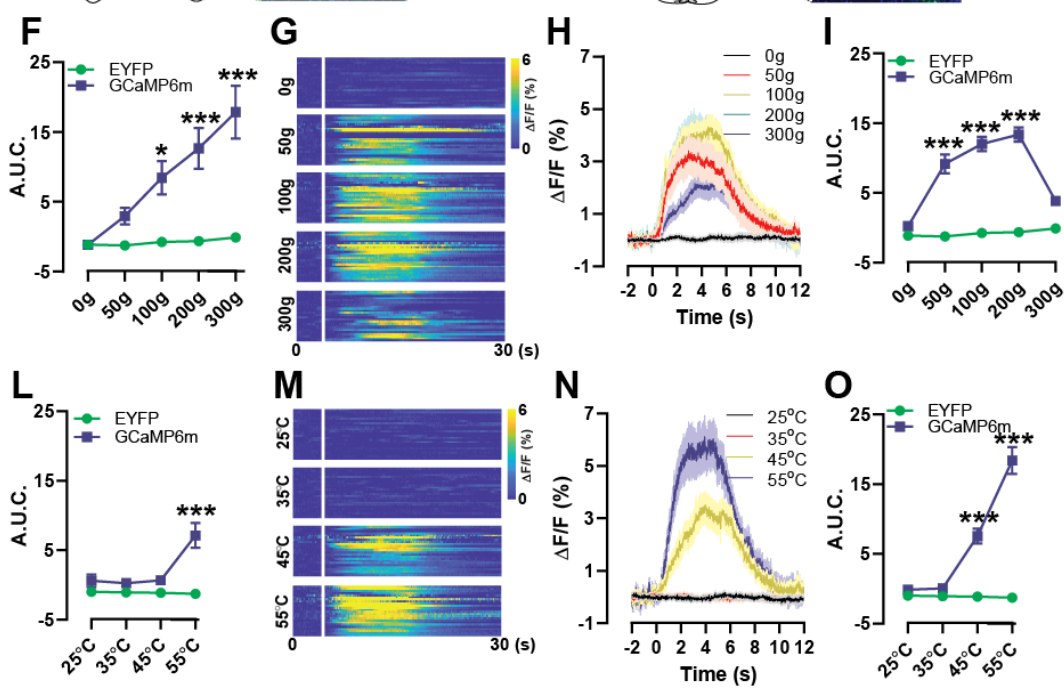

Q $\quad \mathbf{R}$
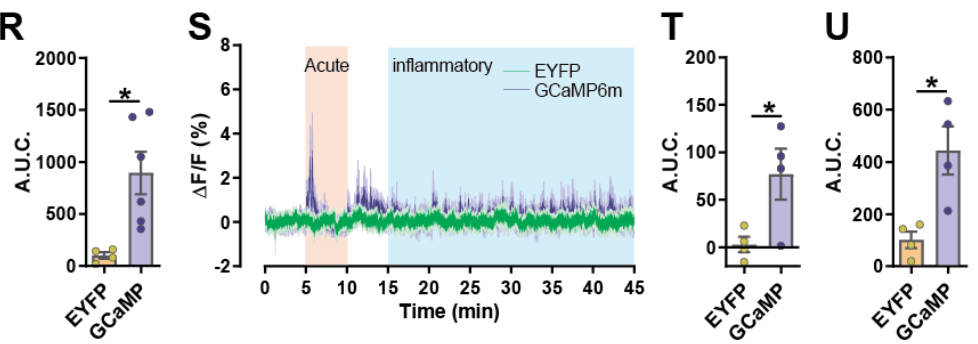
(A) A diagram of the fiber photometry calcium imaging experiment in an anesthetized mouse.

(B and C) A schematic and representative image of the Cre-dependent expression of GCaMP6m in the $\mathrm{Calca}^{\mathrm{Cre}}$ mice with an optical fiber implanted (red dashed line in the overlay image indicates fiber track)

(D-F) Intensity-dependent calcium activity increase in CGRP ${ }^{\mathrm{SPFp}}$ neurons in response to a pressure meter

(G-I) Intensity-dependent calcium activity increase in $\mathrm{CGRP}^{\mathrm{PBel}}$ neurons to a pressure meter.

(J-L) Intensity-dependent calcium increase in CGRP ${ }^{\mathrm{SPF}}$ neurons in response to a temperature-controlled

(M-O) Intensity-dependent calcium activity increase in $\mathrm{CGRP}^{\mathrm{PBel}}$ neurons in response to a temperature-

(P) Calcium signal increases in CGRPSPFp neurons following subcutaneous injection of formalin (4\%) into

(Q) Area under curve (A.U.C.) quantification of the CGRP ${ }^{\mathrm{SPF}}$ neuronal activity during the acute phase (5- 
(R) A.U.C. quantification of the CGRP ${ }^{\mathrm{SPF} p}$ neuronal activity during the inflammatory phase (15-45 min) of formalin injection.

(S) Calcium signal increases in $\mathrm{CGRP}^{\mathrm{PBel}}$ neurons in response to subcutaneous injection of formalin (4\%) into the paw.

(T) A.U.C. quantification of the CGRP ${ }^{\text {PBel }}$ neuronal activity during the acute phase (5-10 min) of formalin response.

(U) A.U.C. quantification of the CGRP ${ }^{\text {PBel }}$ neuronal activity during the inflammatory phase (15-45 min) of formalin response.

\section{Statistics}

(F) Repeated measure two-way ANOVA showed significance in interaction $(\mathrm{F}(4,152)=9.838, \mathrm{p}<0.0001)$, intensity $(\mathrm{F}(4,152)=12.28, \mathrm{p}<0.0001)$ and group $(\mathrm{F}(1,38)=16.01, \mathrm{p}=0.0003) .100(\mathrm{p}<0.05), 200(\mathrm{p}$ $<0.0001)$ and $300 \mathrm{~g}(\mathrm{p}<0.0001)$ points were significantly different between EYFP and GCaMP6m with Sidak's multiple comparisons test.

(I) Repeated measure two-way ANOVA showed significance in interaction $(\mathrm{F}(4,248)=15.08, \mathrm{p}<0.0001)$, intensity $(\mathrm{F}(4,248)=15.17, \mathrm{p}<0.0001)$ and group $(\mathrm{F}(1,62)=64.40, \mathrm{p}<0.0001) .50(\mathrm{p}<0.0001), 100$ $(\mathrm{p}<0.0001)$ and $200 \mathrm{~g}(\mathrm{p}<0.0001)$ points were significantly different between EYFP and GCaMP6m with Sidak's multiple comparisons test.

(L) Repeated measure two-way ANOVA showed significance in interaction $(\mathrm{F}(3,114)=10.97, \mathrm{p}<0.0001)$, intensity $(\mathrm{F}(3,114)=9.61, \mathrm{p}<0.0001)$ and group $(\mathrm{F}(1,38)=11.12, \mathrm{p}=0.0019) .55^{\circ} \mathrm{C}(\mathrm{p}<0.0001)$ was significantly different between EYFP and GCaMP6m with Sidak's multiple comparisons test.

(M) Repeated measure two-way ANOVA showed significance in interaction $(\mathrm{F}(3,186)=23.96, \mathrm{p}<$ $0.0001)$, intensity $(\mathrm{F}(3,186)=22.67, \mathrm{p}<0.0001)$ and $\operatorname{group}(\mathrm{F}(1,62)=46.05, \mathrm{p}<0.0001) .45$ and $55^{\circ} \mathrm{C}$ (both $\mathrm{p}<0.0001$ ) points were significantly different between EYFP and GCaMP6m with Sidak's multiple comparisons test.

(Q) EYFP: $2.86 \pm 8.00$ ( $\mathrm{n}=4$ mice), GCaMP6m: $471.9 \pm 85.53(\mathrm{n}=6$ mice). Unpaired t-test (two-tailed), $\mathrm{p}=0.0024$.

(R) EYFP: $100.60 \pm 31.89(\mathrm{n}=4)$, GCaMP6m: $894.80 \pm 203.40(\mathrm{n}=6)$. Unpaired t-test (two-tailed), $\mathrm{p}=$ 0.0145 .

(K) EYFP: $6.18 \pm 4.05(\mathrm{n}=4)$, GCaMP6m: $77.00 \pm 26.83(\mathrm{n}=4)$. Unpaired t-test (two-tailed), $\mathrm{p}=0.0381$. (L) EYFP: $100.60 \pm 31.89(\mathrm{n}=4)$, GCaMP6m: $443.50 \pm 92.46(\mathrm{n}=4)$. Unpaired t-test (two-tailed), $\mathrm{p}=$ 0.0127 . 

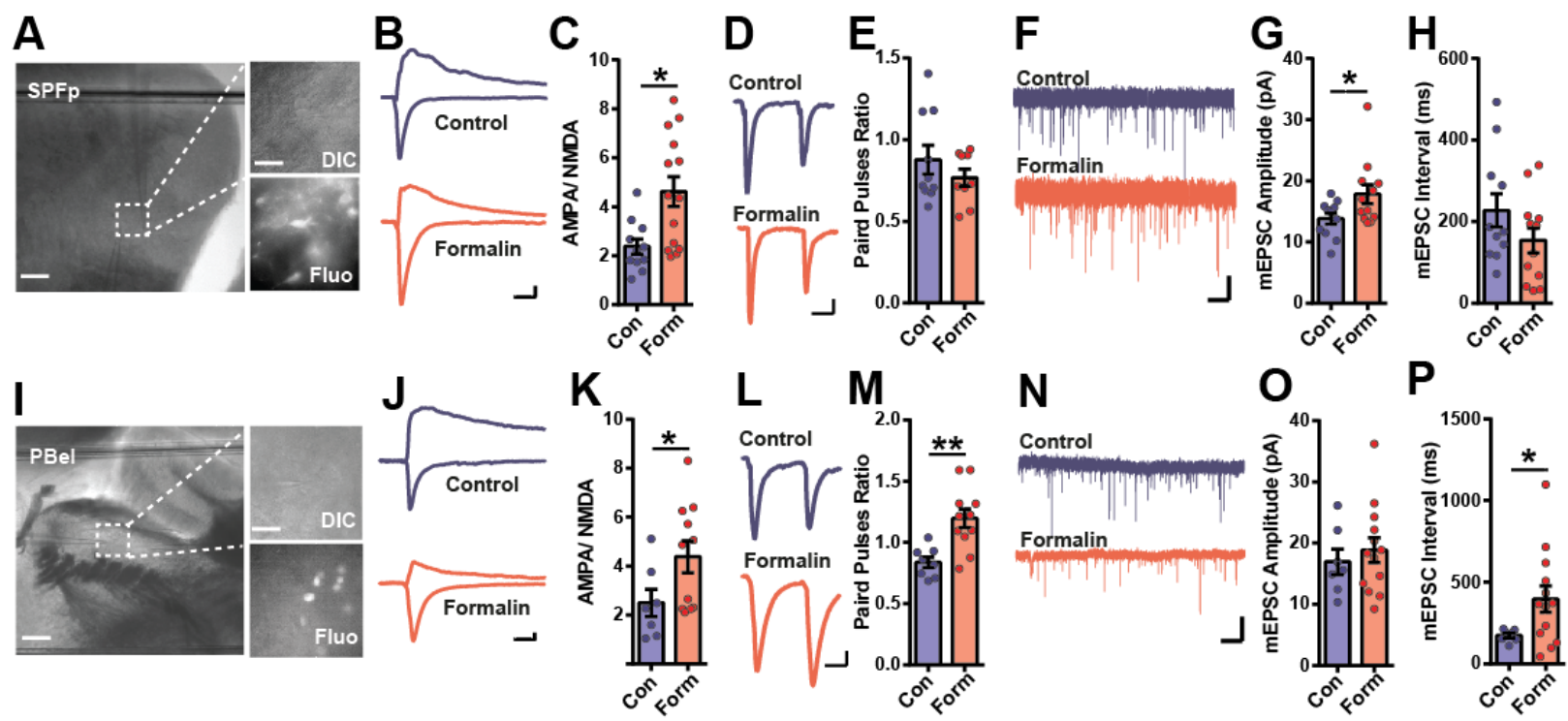

Figure 3. Pain-induced synaptic plasticity changes in CGRP ${ }^{\mathrm{SPFp}}$ and CGRP ${ }^{\mathrm{PBel}}$ neurons. (A) A representative image of a brain slice including SPFp used for the whole-cell patch-clamp experiment. Enlarged images show the fluorescence from Cre:GFP-expressing CGRP neurons. Scale bars, $100 \mu \mathrm{m}$ in left images, and $10 \mu \mathrm{m}$ in enlarged images.

(B) Example traces of AMPA and NMDA EPSCs in control (blue) and formalin (red) injected groups. Scale bars, $20 \mathrm{~ms}$, and $50 \mathrm{pA}$.

(C) The AMPA/NMDA ratio was increased in the formalin injected group compared with the control group. (D and E) No significant differences in the paired-pulse ratio were observed between the formalin-injected group and the control group. Scale bars, $20 \mathrm{~ms}$, and $50 \mathrm{pA}$.

(F) Example traces of mEPSCs in the control (blue) and formalin (red) injected group. Scale bars, $1 \mathrm{~s}$, and $20 \mathrm{pA}$.

(G) mEPSC amplitude was increased in the formalin injected group compared with the control group. (H) mEPSC interval was not changed in the formalin injected group compared with the control group.

(I) A representative image of a brain slice including PBel for the whole-cell patch-clamp experiment. Enlarged images are the PBel cells with and without fluorescence. Cells with fluorescence are CGRP neurons. Scale bars, $100 \mu \mathrm{m}$ in left images, and $10 \mu \mathrm{m}$ in enlarged images.

(J) Example traces of AMPA and NMDA EPSCs in control (blue) and formalin (red) injected groups. Scale bars, $20 \mathrm{~ms}$, and $20 \mathrm{pA}$.

(K) The AMPA/NMDA ratio was increased in the formalin injected group compared with the control group. (L and $\mathbf{M})$ The formalin injected group display an increased paired-pulse ratio compared to the control group. Scale bars, $20 \mathrm{~ms}$, and $50 \mathrm{pA}$.

913 (N) Example traces of mEPSCs in the control (blue) and formalin (red) injected group. Scale bars. $1 \mathrm{~s}$ and $91450 \mathrm{pA}$.

915 (O) mEPSC amplitude was not changed in the formalin injected group compared with the control group. 


\section{Statistics}

922 (C) SPFp; Control: $2.38 \pm 0.31(\mathrm{n}=11$ cells), formalin: $4.62 \pm 0.61(\mathrm{n}=14$ cells). Unpaired $\mathrm{t}$ test (two923 tailed), $\mathrm{p}=0.0063$.

924 (E) SPFp; Control: $0.88 \pm 0.09(\mathrm{n}=10$ cells), formalin: $0.77 \pm 0.05(\mathrm{n}=9$ cells). Unpaired t test (two925 tailed), $\mathrm{p}=0.2957$.

926 (G) SPFp; Control: $13.81 \pm 0.90$ ( $\mathrm{n}=11$ cells), formalin: $17.83 \pm 1.56(\mathrm{n}=12$ cells). Unpaired t test (two927 tailed), $\mathrm{p}=0.0390$.

928 (H) SPFp; Control: $227.4 \pm 40.90(\mathrm{n}=11$ cells), formalin: $154.2 \pm 30.80(\mathrm{n}=12$ cells). Unpaired t test 929 (two-tailed), $\mathrm{p}=0.1687$.

930 (K) PBel; Control: $2.49 \pm 0.56$ ( $\mathrm{n}=7$ cells), formalin: $4.38 \pm 0.65$ ( $\mathrm{n}=11$ cells). Unpaired t test (two-tailed), $931 \mathrm{p}=0.0437$.

932 (M) PBel; Control: $0.84 \pm 0.04$ ( $\mathrm{n}=8$ cells), formalin: $1.20 \pm 0.77(\mathrm{n}=11$ cells). Unpaired t test (two933 tailed), $\mathrm{p}=0.001$.

934 (O) PBel; Control: $16.93 \pm 2.08$ ( $\mathrm{n}=7$ cells), formalin: $18.85 \pm 2.04$ ( $\mathrm{n}=13$ cells). Unpaired t test (two935 tailed), $\mathrm{p}=0.5196$.

936 (P) PBel; Control: $173.2 \pm 13.89$ ( $\mathrm{n}=7$ cells), formalin: $396.5 \pm 80.66$ ( $\mathrm{n}=13$ cells). Unpaired $\mathrm{t}$ test (two937 tailed), $\mathrm{p}=0.0175$. 


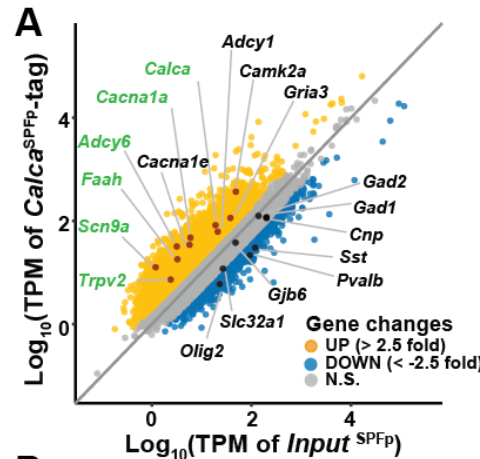

C
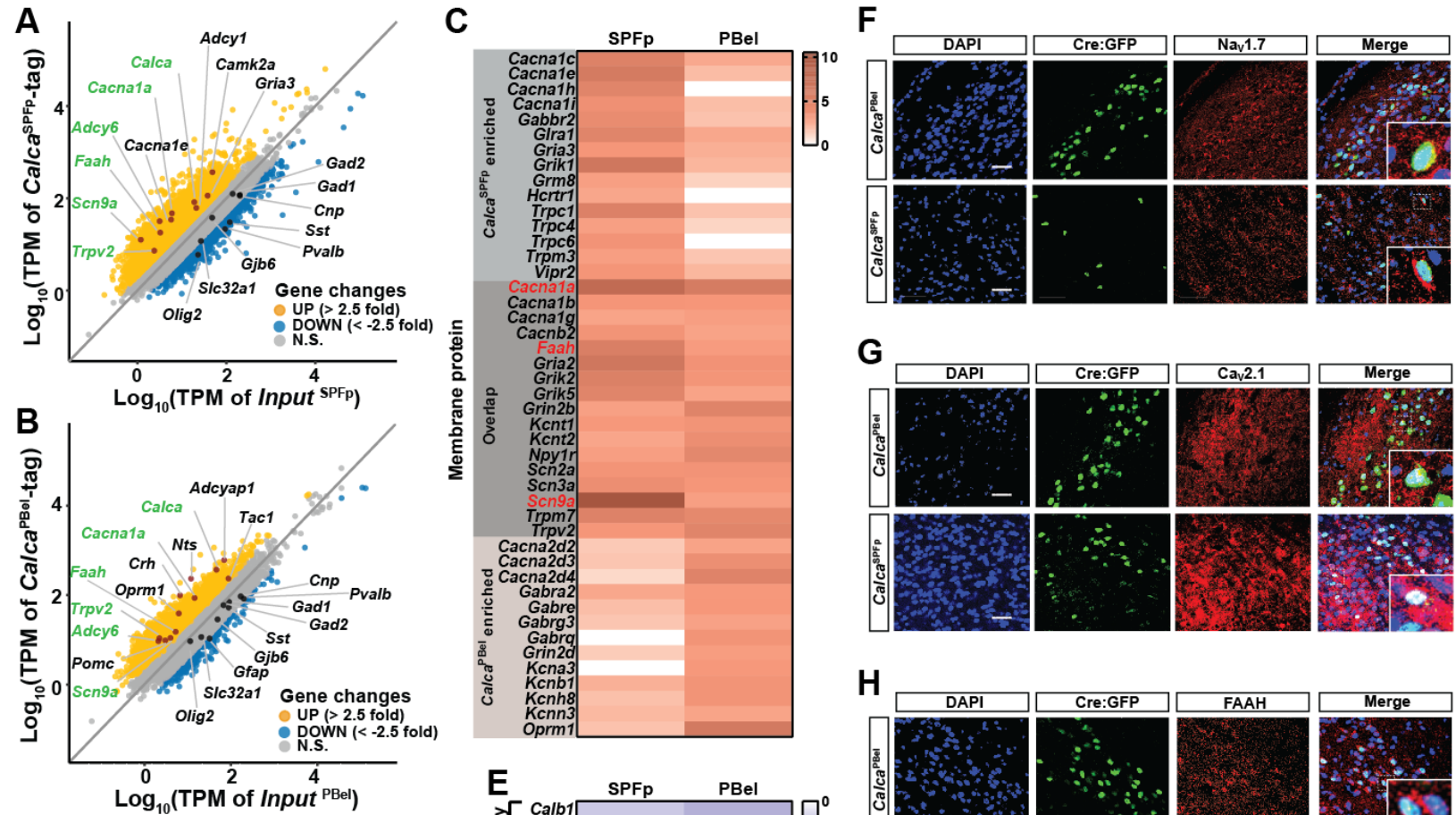

D
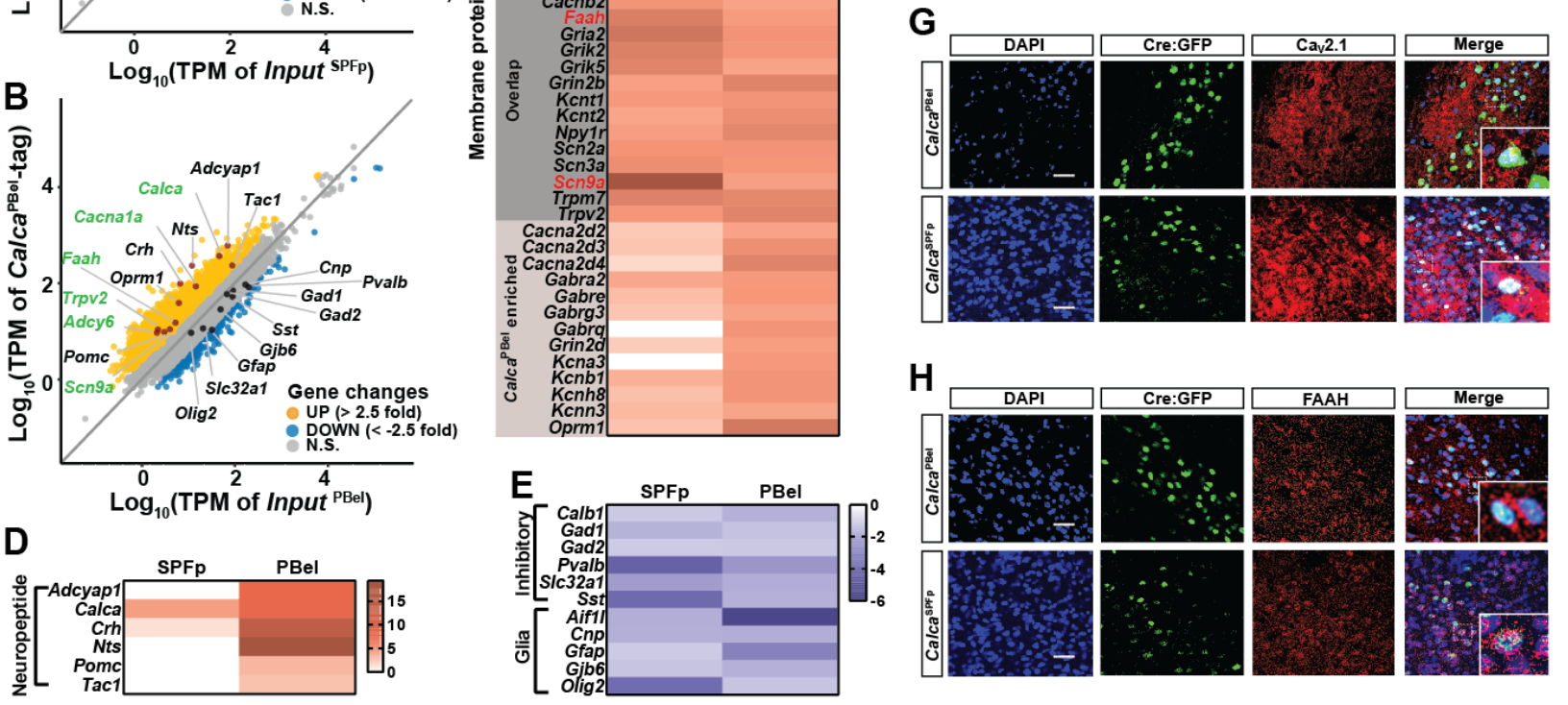

940 Figure 4. Active transcriptome profiling of CGRP ${ }^{\text {SPFp }}$ and CGRP ${ }^{\text {PBel }}$ neurons.

941 (A) Correlation plot showing expression (transcript per million, TPM in log 10 scale) of genes enriched in 942 CGRP $^{\text {SPFp }}$ neurons compared with the total SPFp inputs using RiboTag transcriptome profiling. Up and 943 down-regulated genes were separated based on 2.5 or -2.5 -fold enrichment.

944 (B) Correlation plot of the transcriptome profiles of CGRP ${ }^{\text {PBel }}$ neurons versus total PBel inputs.

945 (C) Heatmaps showing fold changes of genes in the SPFp and PBel that encode membrane proteins.

946 (D) Heatmaps showing fold changes of genes in the SPFp and PBel that encode neuropeptides,

947 (E) Heatmaps showing fold changes of genes in the SPFp and PBel that encode markers of inhibitory neuron 948 or glia.

949 (F-H) Co-expression of Nav1.7 (F), Cav2.1 (G), or FAAH (H) with CGRP by double IHC. Scale bars, 50 $950 \mu \mathrm{m}$. 

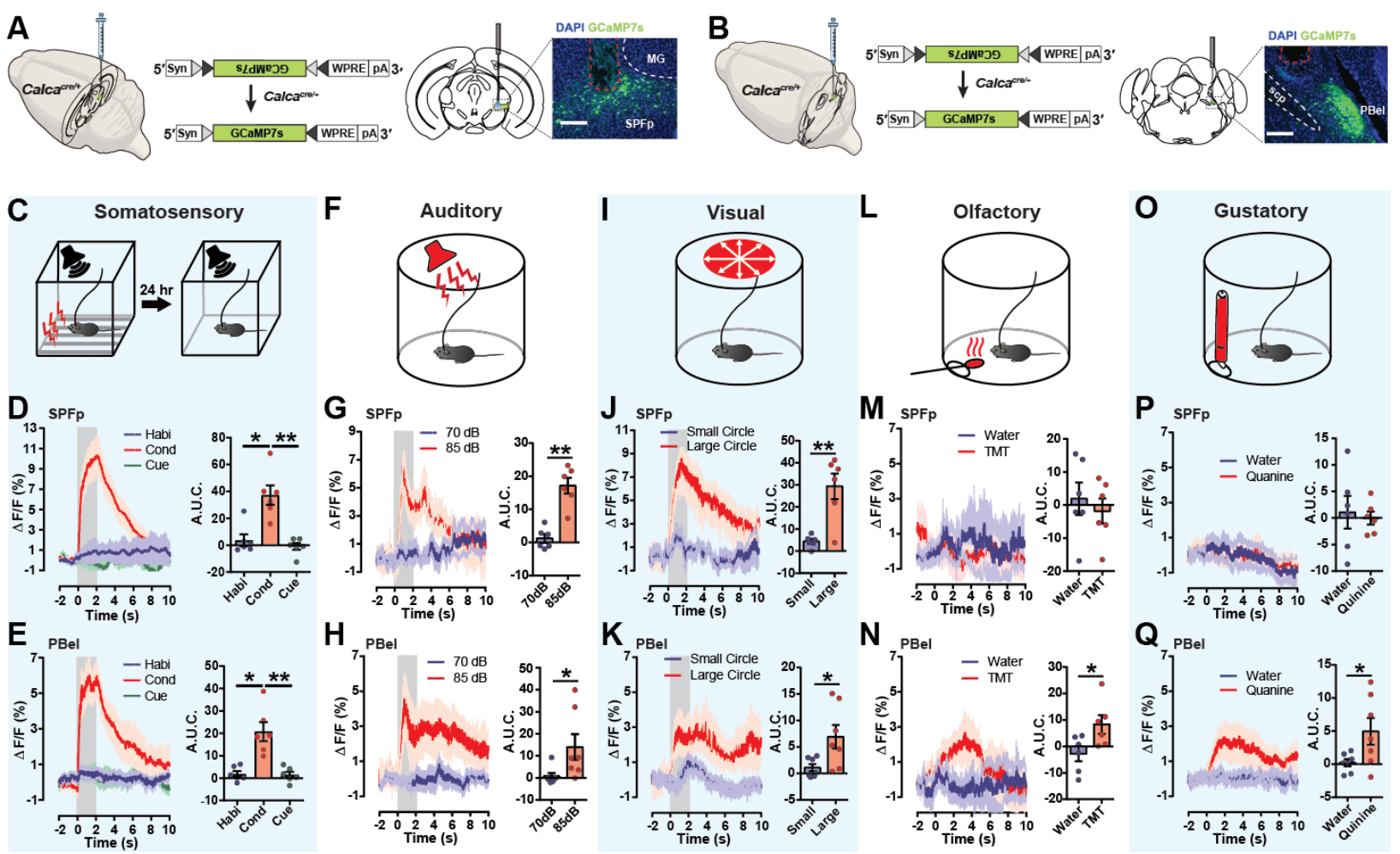

Figure 5. CGRP ${ }^{S P F p}$ and CGRP ${ }^{\text {PBel }}$ neurons are activated by multimodal sensory threat stimuli.

(A and B) A schematic and representative images of Cre-dependent expression of GCaMP7s in the $\operatorname{CGRP}^{\mathrm{SPFp}}(\mathbf{A})$ and CGRP ${ }^{\mathrm{PBel}}(\mathbf{B})$ neurons for fiber photometry. Scale bars, $200 \mu \mathrm{m}$.

(C) Cued fear conditioning test with low volume tone $(72 \mathrm{~dB})$ was performed to examine the responses of CGPR neurons to the somatosensory aversive stimulus (2-s, $0.6 \mathrm{~mA}$ electric foot shock).

(E) neurons were activated by the electric foot shock during the conditioning period, but not habituation, nor the cued retrieval period. Left, averaged calcium traces. Right, A.U.C. quantification.

(F) Intense sound $(85 \mathrm{~dB})$ was used as an aversive auditory stimulus, with a70-dB low-intensity sound as a control.

(G and $\mathbf{H}) \operatorname{CGRP}^{\mathrm{SPF}}(\mathbf{G})$ and $\mathrm{CGRP}^{\mathrm{PBel}}(\mathbf{H})$ neurons were activated by the intense sound. Left, averaged calcium traces. Right, A.U.C. quantification. (I) A large looming disk was used as an aversive visual stimulus, with a small disk as a control. (J and K) CGRP ${ }^{\text {SPFp }}(\mathbf{J})$ and CGRP ${ }^{\text {PBel }}(\mathbf{K})$ neurons were activated by the large looming disk. Left, averaged calcium traces. Right, A.U.C. quantification.

(L) TMT-soaked cotton was used as an aversive olfactory stimulus, with water as a control.

(M) There was no activity change in CGRP ${ }^{\mathrm{SPF}}$ neurons when the animal approached the TMT-soaked cotton. Left, averaged calcium traces. Right, A.U.C. quantification.

(N) CGRP ${ }^{\mathrm{PBel}}$ neurons were activated when the animal approached the TMT-soaked cotton. Left, averaged

972 (O) Quinine solution $(0.5 \mathrm{mM})$ was introduced as an aversive gustatory stimulus (water was the control).

973

974

(P) Quinine did not affect CGRP ${ }^{S P F}$ neurons. Left, averaged calcium traces. Right, A.U.C. quantification.

975

(Q) CGRP ${ }^{\text {PBel }}$ neurons were activated by quinine solution $(0.5 \mathrm{mM})$. Left, averaged calcium traces. Right, 


\section{Statistics}

978 (D) SPFp; Habituation: $3.63 \pm 4.45$, conditioning: $37.31 \pm 7.18$, cue test: $-0.92 \pm 2.57(\mathrm{n}=6)$. Repeated

979 measure one-way ANOVA, $\mathrm{p}=0.0029$. Conditioning was significantly different with habituation $(\mathrm{p}<0.05)$

980 and cue test ( $\mathrm{p}<0.01$, Tukey's multiple comparisons).

981 (E) PBel; SPFp; Habituation: $1.85 \pm 1.33$, conditioning: $20.73 \pm 4.25$, cue test: $1.49 \pm 1.35(\mathrm{n}=6)$. Repeated

982 measure one-way ANOVA, $\mathrm{p}=0.0022$. Conditioning was significantly different with habituation $(\mathrm{p}<0.05)$

983 and cue test ( $\mathrm{p}<0.01$, Tukey's multiple comparisons).

984 (G) SPFp; $70 \mathrm{~dB}: 1.226 \pm 1.27,85 \mathrm{~dB}: 17.14 \pm 2.38(\mathrm{n}=6)$. Paired t test (two-tailed), $\mathrm{p}=0.0018$.

985 (H) PBel; $70 \mathrm{~dB}: 0.78 \pm 1.46,85 \mathrm{~dB}: 14.06 \pm 5.86(\mathrm{n}=7)$. Paired t test (two-tailed), $\mathrm{p}=0.0437$.

986 (J) SPFp; Small disk: $4.41 \pm 1.11$, large disk: $29.30 \pm 5.81(\mathrm{n}=6)$. Paired t test (two-tailed), $\mathrm{p}=0.0091$.

987 (K) PBel; Small disk: $1.08 \pm 0.69$, large disk: $6.93 \pm 2.23(\mathrm{n}=7)$. Paired t test (two-tailed), $\mathrm{p}=0.0357$.

988 (M) SPFp; Water: $1.93 \pm 4.88$, TMT: $-1.82 \pm 3.78(\mathrm{n}=6)$. Paired t test (two-tailed), $\mathrm{p}=0.4411$.

989 (N) PBel; Water: $-2.72 \pm 2.84$, TMT: $8.28 \pm 3.57(\mathrm{n}=6)$. Paired t test (two-tailed), $\mathrm{p}=0.0145$.

990 (P) SPFp; Water: $1.10 \pm 3.09$, quinine: $-0.01 \pm 1.22(\mathrm{n}=6)$. Paired t test (two-tailed), $\mathrm{p}=0.7707$.

991 (Q) PBel; Water: 0.2209 \pm 0.83, quinine: $4.96 \pm 2.01(\mathrm{n}=7)$. Paired t test (two-tailed), $\mathrm{p}=0.0377$. 


\section{A}
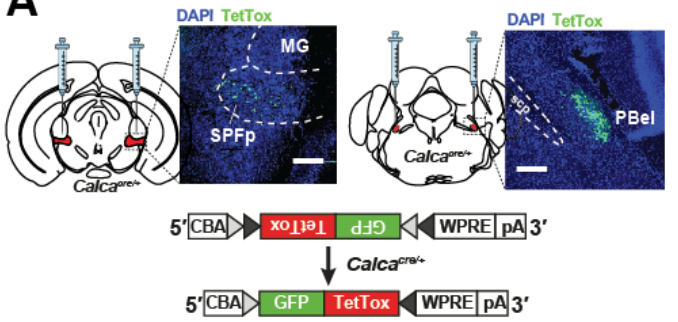
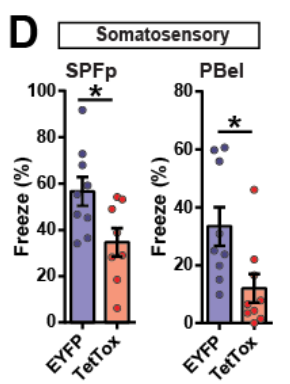

E

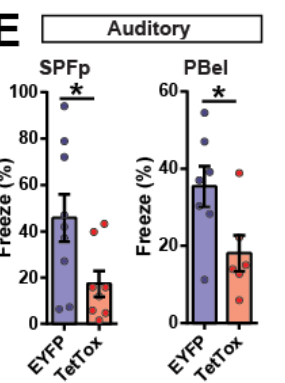

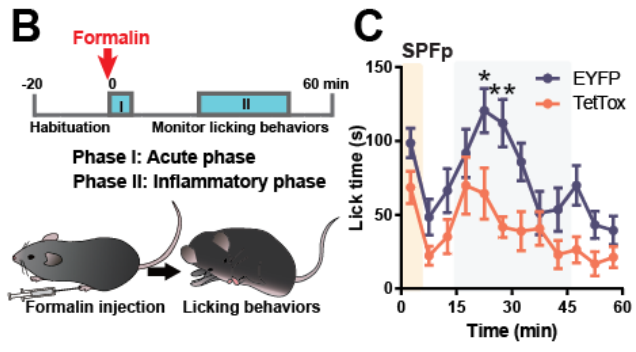

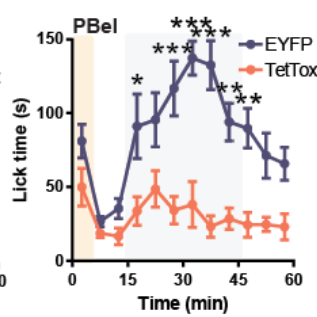

$\mathbf{F}$

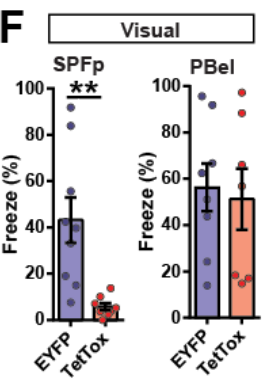

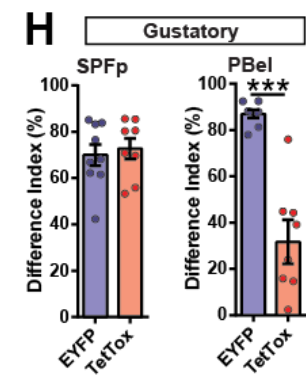

Figure 6. Silencing CGRP ${ }^{\mathrm{SPF} p}$ or CGRP ${ }^{\mathrm{PBel}}$ neuronal activities attenuates perception of affective pain and multisensory threat stimuli.

(A) Schematics and representative images of Cre-dependent TetTox expression targeting CGRP ${ }^{\mathrm{SPF}}$ or CGRP ${ }^{\text {PBel }}$ neurons. Scale bars, $200 \mu \mathrm{m}$.

(B) A schematic diagram of the Formalin assay to test inflammatory pain responses.

(C) The CGRP ${ }^{\mathrm{SPF}}$ and $\mathrm{CGRP}^{\mathrm{PBel}}$ silenced groups displayed significantly alleviated inflammatory pain responses.

(D) Both the CGRPSPFp and CGRP ${ }^{\text {PBel }}$ silenced groups froze less in response to electric foot shock (2-s, 0.6 $\mathrm{mA})$ compared to controls.

(E) Both the CGRP ${ }^{\mathrm{SPF}}$ and $\mathrm{CGRP}^{\mathrm{PBel}}$ silenced groups froze less in response to $85-\mathrm{dB}$ intense sound compared with controls.

(F) In response to a looming aversive visual stimulus, the CGRP ${ }^{S P F p}$ silenced group showed less freezing, whereas there was no difference between EYFP and CGRP ${ }^{\text {PBel }}$ silenced groups.

(G) A two-chamber choice test with TMT- and water-soaked cotton placed at each corner of the chamber was used to test animals' responses to aversive olfactory stimulus. The CGRP ${ }^{\mathrm{SPF}}$ silenced group, and EYFP controls both avoided the TMT chamber, while the CGRP ${ }^{\mathrm{PBel}}$ TetTox group exhibited less aversion to TMT, spending equal amounts of time in the water and TMT chambers.

(H) A quinine-water choice test was performed to test the animals' responses to the aversive gustatory stimulus (0.5 mM quinine solution). There was no change in water preference in the CGRP ${ }^{\mathrm{SPFp}}$ silenced group, but the $\mathrm{CGRP}^{\mathrm{PBel}}$ silenced group showed a lower difference index than the control group.

\section{Statistics}

(C) SPFp: Repeated measure two-way ANOVA showed no significance in interaction $(\mathrm{F}(11,187)=1.32$, $\mathrm{p}=0.2148)$, but in time $(\mathrm{F}(11,187)=9.05, \mathrm{p}<0.0001)$ and group $(\mathrm{F}(1,17)=9.57, \mathrm{p}=0.0066) .20-25(\mathrm{p}$ $<0.05)$ and 25-30 ( $<$ 0.01) min point were significantly different between EYFP and TetTox with Sidak's multiple comparisons test.

PBel: Repeated measure two-way ANOVA showed significance in interaction $(\mathrm{F}(11,187)=3.45, \mathrm{p}=$ $0.0002)$, time $(\mathrm{F}(11,187)=5.97, \mathrm{p}<0.0001)$ and group $(\mathrm{F}(1,17)=53.24, \mathrm{p}<0.0001) .15-20(\mathrm{p}<0.05)$, 
25-30 ( $\mathrm{p}<0.0001), 30-35$ ( $\mathrm{p}<0.0001), 35-40$ ( $\mathrm{p}<0.0001), 40-45(\mathrm{p}<0.01), 45-50(\mathrm{p}<0.01) \min$ point were significantly different between EYFP and TetTox with Sidak's multiple comparisons test.

1025 (D) SPFp; EYFP: $56.62 \pm 6.21 \%(\mathrm{n}=9)$, TetTox: $34.65 \pm 6.11 \%(\mathrm{n}=8)$. Unpaired t test (two-tailed), $\mathrm{p}=$ 10260.0240.

1027 PBel; EYFP: $33.43 \pm 6.66 \%(\mathrm{n}=9)$, TetTox: $12.04 \pm 4.88 \%(\mathrm{n}=9)$. Unpaired t test (two-tailed), $\mathrm{p}=$ 10280.0197.

1029 (E) SPFp; EYFP: $45.74 \pm 10.26 \%(\mathrm{n}=9)$, TetTox: $17.32 \pm 5.58 \%(\mathrm{n}=8)$. Unpaired t test (two-tailed), $\mathrm{p}$ $1030=0.0332$.

1031 PBel; EYFP: $35.39 \pm 5.29 \%(\mathrm{n}=7)$, TetTox: $18.14 \pm 4.66 \%(\mathrm{n}=6)$. Unpaired t test (two-tailed), $\mathrm{p}=$ 10320.0350.

1033 (F) SPFp; EYFP: $43.12 \pm 9.82 \%(\mathrm{n}=9)$, TetTox: $5.73 \pm 1.57 \%(\mathrm{n}=8)$. Unpaired $\mathrm{t}$ test (two-tailed), $\mathrm{p}=$ 10340.0029.

1035 PBel; EYFP: $56.20 \pm 10.33 \%(\mathrm{n}=8)$, TetTox: $51.22 \pm 13.21 \%(\mathrm{n}=7)$. Unpaired t test (two-tailed), $\mathrm{p}=$ 10360.0029.

1037 (G) SPFp; EYFP: $20.12 \pm 4.91 \%(\mathrm{n}=9)$, TetTox: $15.55 \pm 7.75 \%(\mathrm{n}=8)$. Unpaired $\mathrm{t}$ test (two-tailed), $\mathrm{p}=$ 10380.6175.

1039 PBel; EYFP: $13.87 \pm 3.94 \%(\mathrm{n}=11)$, TetTox: $-1.53 \pm 5.89 \%(\mathrm{n}=10)$. Unpaired $\mathrm{t}$ test (two-tailed), $\mathrm{p}=$ $1040 \quad 0.0396$.

1041 (H) SPFp; EYFP: $69.96 \pm 4.64 \%(\mathrm{n}=9)$, TetTox: $72.66 \pm 4.48 \%(\mathrm{n}=8)$. Unpaired $\mathrm{t}$ test (two-tailed), $\mathrm{p}=$ 10420.6823.

1043 PBel; EYFP: $86.87 \pm 1.77 \%(\mathrm{n}=8)$, TetTox: $31.66 \pm 9.45 \%(\mathrm{n}=7)$. Unpaired t test (two-tailed), $\mathrm{p}<$ 10440.0001. 

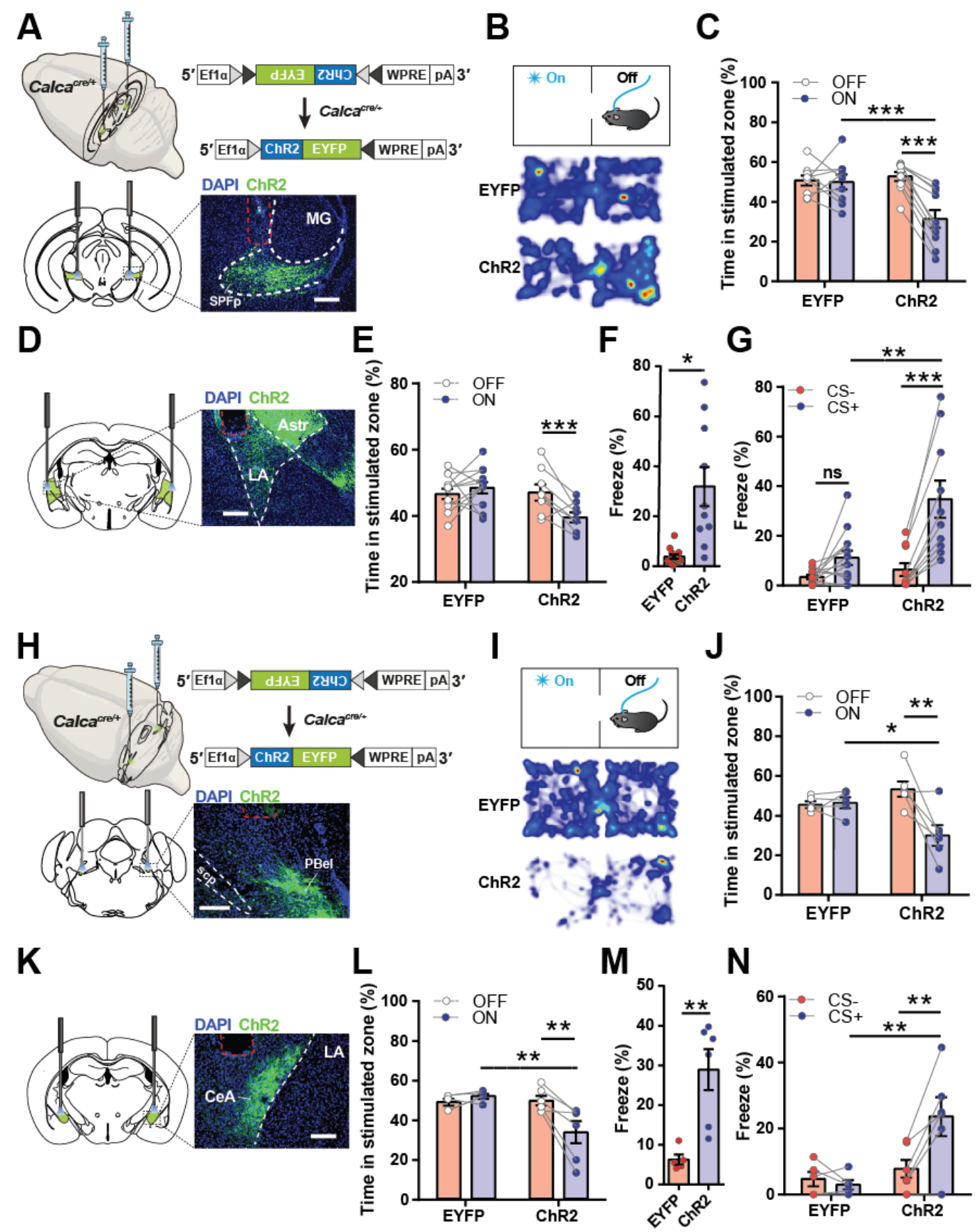

Figure 7. Activating the CGRP ${ }^{\mathrm{SPF} \rightarrow \mathrm{LA}}$ or $\mathrm{CGRP}^{\mathrm{PBel} \rightarrow \mathrm{CeA}}$ pathways encodes negative valence.

(A) Schematics and representative image of Cre-dependent expression of ChR2 in the SPFp of Calca ${ }^{\text {Cre }}$ 1049 mice and optic fiber placement (red dotted line).

(B) A schematic diagram and heatmap of the real-time place aversion (RTPA) test with CGRP ${ }^{\mathrm{SPF}}$ cell body stimulation.

1052 (C) Time spent in the stimulated zone during the RTPA test.

1053 (D) Representative image of optic fiber placement in the LA for terminal stimulation (red dotted line).

1054 (E) Time spent in the stimulated zone during the RTPA test with CGRP ${ }^{\mathrm{SPFp} \rightarrow \mathrm{LA}}$ terminal stimulation.

1055 (F) Time spent freezing during the context test. 
1056

1057

1058

1059

1060

1061

1062

1063

1064

1065

1066

1067

1068

1069

1070

1071

1072

1073

1074

1075

1076

1077

1078

1079

1080

1081

1082

1083

1084

1085

1086

1087

1088

1089

1090

1091

1092

1093

1094

1095

1096

(G) Time spent freezing during cue test after pairing optic stimulation and tone for cued fear conditioning. (H) Schematic and representative image of Cre-dependent expression of ChR2 in the PBel of Calca ${ }^{\mathrm{Cre}}$ mice and optic fiber placement (red dotted line).

(I) Schematic diagram and heatmap of real-time place aversion (RTPA) test with CGRP ${ }^{\text {PBel }}$ cell body stimulation.

(J) Time spent in the stimulated zone during the RTPA test with CGRP ${ }^{\text {PBel }}$ cell body stimulation.

(K) Representative image of optic fiber placement in the CeA (red dot).

(L) Time spent in the stimulated zone during the RTPA test with CGRP ${ }^{\mathrm{PBel} \rightarrow \mathrm{CeA}}$ terminal stimulation.

(M) Time spent freezing during the context test.

(N) Time spent freezing during cue test after pairing optogenetic stimulation and tone for cued fear conditioning. Scale bars indicate $200 \mu \mathrm{m}$.

\section{Statistics}

(C) Repeated measure two-way ANOVA showed significance in laser x group interaction $(\mathrm{F}(1,17)=17.69$, $\mathrm{p}=0.0006)$ and laser $(\mathrm{F}(1,17)=20.19, \mathrm{p}=0.0003)$ but not group $(\mathrm{F}(1,17)=4.03, \mathrm{p}=0.0608)$. Laser ON and OFF in ChR2 ( $\mathrm{p}<0.0001)$, and EYFP and ChR2 during ON $(\mathrm{p}<0.001)$ had statistically significant difference with Sidak's multiple comparisons test.

(E) Repeated measure two-way ANOVA showed significance in only laser $\mathrm{x}$ group interaction $(\mathrm{F}(1,18)=$ $8.718, \mathrm{p}=0.0085)$ but not laser $(\mathrm{F}(1,18)=3.159 \mathrm{p}=0.0924)$ and group $(\mathrm{F}(1,18)=4.343, \mathrm{p}=0.0517)$. Laser ON vs OFF in ChR2 ( $p<0.05)$, and EYFP vs ChR2 during ON ( $<<0.01)$ have statistically significant difference with Sidak's multiple comparisons test.

(F) EYFP: $3.842 \pm 0.89(\mathrm{n}=13)$, ChR2: $31.92 \pm 7.806(\mathrm{n}=10)$. Unpaired t test (two-tailed), $\mathrm{p}=0.0005$.

(G) Repeated measure two-way ANOVA showed significance in CS x group interaction $(\mathrm{F}(1,21)=12.41$, $\mathrm{p}=0.0020), \mathrm{CS}(\mathrm{F}(1,21)=38.26, \mathrm{p}<0.0001)$ and group $(\mathrm{F}(1,21)=8.392, \mathrm{p}=0.0086)$. CS- vs CS+ in ChR2 ( $\mathrm{p}<0.0001)$, and EYFP vs ChR2 during CS+ $(\mathrm{p}<0.001)$ have statistically significant difference with Sidak's multiple comparisons test.

(J) Repeated measure two-way ANOVA showed significance in laser x group interaction $(\mathrm{F}(1,9)=10.49$, $\mathrm{p}=0.0102)$ and laser $(\mathrm{F}(1,9)=8.959, \mathrm{p}=0.0151)$ but not group $(\mathrm{F}(1,9)=1.159, \mathrm{p}=0.3096)$. Laser ON and OFF in ChR2 ( $\mathrm{p}<0.01)$, and EYFP and ChR2 during ON $(\mathrm{p}<0.05)$ had statistically significant difference with Sidak's multiple comparisons test.

(L) Repeated measure two-way ANOVA showed significance in laser x group interaction $(\mathrm{F}(1,9)=12.91$, $\mathrm{p}=0.0058)$ and laser $(\mathrm{F}(1,9)=6.094, \mathrm{p}=0.0357)$ but not in group $(\mathrm{F}(1,9)=4.405, \mathrm{p}=0.0652)$. Laser ON and OFF in ChR2 ( $p<0.01)$, and EYFP and ChR2 during ON $(\mathrm{p}<0.01)$ had statistically significant difference with Sidak's multiple comparisons test.

(M) EYFP: $6.278 \pm 1.244(\mathrm{n}=5)$, ChR2: $28.93 \pm 5.133(\mathrm{n}=6)$. Unpaired t test (two-tailed), $\mathrm{p}=0.0035$.

(N) Repeated measure two-way ANOVA showed significance in CS x group interaction $(\mathrm{F}(1,9)=12.67$, $\mathrm{p}=0.0061), \mathrm{CS}(\mathrm{F}(1,9)=8.226, \mathrm{p}=0.0185)$ and group $(\mathrm{F}(1,9)=6.314, \mathrm{p}=0.0332)$. CS- vs CS+ in ChR2 ( $p<0.01)$, and EYFP vs ChR2 during CS+ $(\mathrm{p}<0.01)$ have statistically significant difference with Sidak's multiple comparisons test. 


\section{Supplementary Information}

1098

1099

1100

1101

1102

1103

1104

1105

1106

1107

1108

1109

1110

1111

1112

1113

1114

1115

1116

1117

1118

1119

1120

1121

1122

1123

1124

1125

1126

1127

1128

1129

1130

1131

1132

1133

1134

1135

1136

1137

1138

1139

1140

\section{Materials and methods Animals}

All protocols for animal experiments were approved by the IACUC of the Salk Institute for Biological Studies according to NIH guidelines for animal experimentation. The $\mathrm{Calca}^{\mathrm{Cre}}$, and $\mathrm{Tacrl}^{\mathrm{Cre}}$ transgenic mouse lines used in this study were generated from the Richard Palmiter's laboratory (Han et al., 2015 or Carter et al., 2013) $C d x 2^{F l p O}$ line was generated from the Martyn Goulding's laboratory. Calca ${ }^{\text {CreER }}$ mouse line was generated from the Pao-Tien Chuang's laboratory. RiboTag Rpl22 ${ }^{H A / H A}$ (Stock No. 011029) and Ai65 (Stock No: 021875) mouse line was obtained from the Jackson Laboratory. All mouse lines are backcrossed with C57B1/6J for $>6$ generations. Male and female mice were used in all studies. Animals were randomized to experimental groups and no sex differences were noted. Mice were maintained on a normal 12 hours light/dark cycle and provided with food and water ad libitum.

\section{Stereotaxic surgery for virus injection and optic fiber implantation}

Mice were anesthetized by isoflurane gas anesthesia (induction at 3.5\%, and maintenance at $1.5-2 \%$, the Dräger Vapor ${ }^{\circledR} 2000$; Draeger, Inc., USA). Mice were then placed on a stereotaxic frame (David Kopf Instruments, USA). Holes were drilled with a micromotor handpiece drill (Foredom, USA) after the exposure of the skull. The virus was injected using a syringe (65458-01, Hamilton, USA) connected to an ultra-micropump (UMP-3, World Precision Instruments, USA). Unilateral (right side) and bilateral injections were made for the following target regions: SPFp (antero-posterior (AP), -3.1 mm; medio-lateral (ML), $2.0 \mathrm{~mm}$; dorso-ventral (DV) -3.6 mm from bregma) or PBel (AP, -5.1 mm; ML, $1.35 \mathrm{~mm}$; DV, -3.5 $\mathrm{mm}$ ). Viruses were injected at a rate of $0.08 \mu \mathrm{l} / \mathrm{min}$ (total volume of $0.75 \mu \mathrm{l}$ for optogenetic projection studies and $0.5 \mu \mathrm{l}$ for all the others) and the syringe needle was slowly removed from the injection site seven-minute after injection. To determine the inputs to CGRP ${ }^{\mathrm{SPF}}$ and CGRP ${ }^{\mathrm{PBel}}$ neurons, $0.5 \mu 1$ of AAV8hSyn-FLEX-TVA-P2A-GFP-2A-oG (Salk Institute viral vector core, USA) was injected into the SPFp or PBel of $\mathrm{Calca}^{\mathrm{Cre}}$ transgenic mice. After three weeks, $0.5 \mu \mathrm{l}$ of EnvA- $\Delta$ G-rabies-mCherry (Salk Institute viral vector core, USA) was injected, and the mice were sacrificed five days after the injection. To silence the CGRP ${ }^{\mathrm{SPF}}$ and CGRP ${ }^{\mathrm{PBel}}$ neurons, $0.5 \mu \mathrm{l}$ of AAV-DIO-TetTox-GFP or AAV-DIO-EYFP was injected into the SPFp or PBel of $\mathrm{Calca}^{\mathrm{Cre}}$ transgenic mice, and experiments were performed two weeks after injection. For fiber photometry experiments, mice were injected with $0.5 \mu \mathrm{l}$ of either AAV-DIO-GCaMP6m, AAV-DIO-GCaMP7s or AAV-DIO-EYFP into the SPFp or PBel of Calca ${ }^{\text {Cre }}$ mice. Stainless-steel mono fiberoptic cannulas (400 um diameter, 0.37 NA, Doric Lenses) were implanted above the SPFp or PBel. For electrophysiology, mice were injected with 0.5 or $0.75 \mu$ l of AAV1-DIO-ChR2-EYFP into the SPFp or PBel of $\mathrm{Calca}^{\mathrm{Cre}}$ mice. Experiments were performed two weeks after viral injection for recording SPFp and PBel neurons or four weeks after injection for recording cells in terminal regions. For optogenetics, mice were injected with 0.5 or $0.75 \mu$ l of AAV1-DIO-ChR2-EYFP or AAV-DIO-EYFP into the SPFp or PBel of $\mathrm{Calca}^{\mathrm{Cre}}$ mice and custom made mono fiberoptic cannula (200 um diameter, $0.22 \mathrm{NA}$ ) were implanted above SPFp (0.5 mm above the injection site), PBel ( $0.5 \mathrm{~mm}$ above the injection site), Astr (AP, $-1.8 \mathrm{~mm}$; ML, $3.3 \mathrm{~mm}$, DV, -3.8 mm from bregma), lateral amygdala (LA; AP, -1.8 mm; ML, $3.6 \mathrm{~mm}, \mathrm{DV},-4.0 \mathrm{~mm}$ from bregma), pIC (AP, -1.5 mm; ML, $4.6 \mathrm{~mm}$, DV, -3.0 mm from bregma) or central amygdala (CeA; AP, $1.2 \mathrm{~mm}$; ML, $2.7 \mathrm{~mm}$; DV, $-4.2 \mathrm{~mm}$ from bregma). Experiments were executed two weeks after injection to manipulate SPFp neurons or four weeks later for terminal stimulations. 


\section{Histology and quantification of rabies tracing experiment}

1142 Mice were intracardially perfused with 4\% paraformaldehyde in PBS 5 days after the rabies virus injection. 1143 Spinal cords were post-fixed at $4^{\circ} \mathrm{C}$ for $1 \mathrm{~h}$ and dehydrated with $30 \%$ sucrose at $4^{\circ} \mathrm{C}$ overnight. 40 um transverse sections were obtained with a cryostat (CM 1950, Leica, USA) throughout the spinal cord. Spinal cord slices were directly dry mounted on superfrost plus microscope slide glasses (12-550-15, Fisher Scientific, USA). The labeled neurons were counted manually by dividing the transverse spinal sections into four groups (cervical, thoracic, lumbar, and sacral) or different dorsal horn layers. Brains were kept in $4 \%$ PFA overnight for post-fixation and dehydrated in 30\% sucrose for 1-2 days before sectioning. Frozen brains were cut into $50 \mu \mathrm{m}$ coronal slices with a cryostat and stored in Phosphate buffered saline before mounting. Both spinal cord and brain tissues were mounted on a slide glass with a DAPI containing mounting solution (0100-20, SouthernBiotech, USA).

\section{Fiber photometry}

Bulk calcium signals from the CGRP ${ }^{\mathrm{SPF}}$ and $\mathrm{CGRP}^{\mathrm{PBel}}$ neurons were monitored using a custom-built fiber

photometry system based on the open-source pyPhotometry platform (https://pyphotometry.readthedocs.io/en/latest/). $465 \mathrm{~nm}$ LED was used to induce $\mathrm{Ca}^{2+}$ dependent fluorescence signals, and $405 \mathrm{~nm}$ LED was used for $\mathrm{Ca}^{2+}$ independent (isosbestic) fluorescence signals. Motion corrected $\Delta \mathrm{F} / \mathrm{F}$ was calculated by a post-hoc analysis $\left(\Delta \mathrm{F} / \mathrm{F}=\mathrm{F}_{465}-\mathrm{F}_{405 \mathrm{fit}} / \mathrm{F}_{405 \mathrm{fit}}\right)$. The least-squares polynomial function was used to calculate $\mathrm{F}_{405 \mathrm{fit}}$ and the area under the curve was used to analyze the data.

\section{Multi-modal aversive stimuli experiments}

Mechanical and thermal stimuli were applied to mice forepaw, hind paw, or tail. Mechanical pressure was applied using a dial tension gauge (ATG-300-1, Vetus Instruments, USA) with stimulus strength 0, 50, 100, $200 \mathrm{~g}$. The thermal stimulus was applied using a custom-made temperature-controlled hot-rod (TA4SNR+K, Mypin, China) at 25, 35, 45, and $55^{\circ} \mathrm{C}$. A stable baseline was recorded first for $10 \mathrm{~s}$, and stimuli were applied immediately after 5 seconds.

\section{Formalin test}

1170

For fiber photometry, lightly anesthetized mice were placed in the stereotaxic frame head fixed to minimize movement. $10 \mu \mathrm{l}$ of $4 \%$ formalin (1.6\% Paraformaldehyde, 19210, Electron Microscopy Sciences, USA) was injected subcutaneously on the contralateral forepaw after at least $5 \mathrm{~min}$ of stable baseline. Calcium transients were recorded for $45 \mathrm{~min}$. For the loss of function experiment, $10 \mu 1$ of $4 \%$ formalin was injected subcutaneously on one side of the forepaw. Mice were then placed in a Plexiglas chamber $(10 \times 10 \times 13$ $\mathrm{cm}$ ) with a mirror placed behind. Behaviors were recorded for an hour, and licking behaviors were manually counted throughout the experiment.

\section{Auditory and visual stimuli}

For both auditory and visual stimuli experiments, mice were placed in a cylinder-shaped arena $(11 \mathrm{~cm}$ diameter with $15 \mathrm{~cm}$ height) with homecage bedding and were habituated for 30-120 min. For auditory experiments, after a stable $10 \mathrm{~s}$ baseline, an intense sound $(85 \mathrm{~dB}, 2 \mathrm{~s})$ or a control sound $(70 \mathrm{~dB})$ was played. For the loss-of-function experiments, mice were placed inside an open field chamber and were habituated for $10 \mathrm{~min}$. After 1 min baseline, an intense sound $(85 \mathrm{~dB}, 2 \mathrm{~s})$ was delivered three times with an interstimulus interval of $28 \mathrm{~s}$. All the trials were recorded by a USB camera (DFK 33GX236, Imagine Source, Germany) attached to a computer, and freezing behavior was analyzed using video-tracking software (Ethovision XT, Noldus, Netherlands). For visual looming experiments, after a stable $10 \mathrm{~s}$ baseline, an 
expanding looming stimulus ( $2 \mathrm{~s}$ ) was delivered three times with $10 \mathrm{~s}$ inter-stimulus interval with a LED screen facing the arena from above. For the loss-of-function experiment, mice were placed in a cage with bedding and were positioned under the same LED screen. Mice were habituated for 20-30 min. When mice were in the center, the expanding looming stimulus ( $2 \mathrm{~s})$ was delivered three times with $10 \mathrm{~s}$ inter-stimulus interval. All the trials were recorded by a USB camera (DFK 33GX236, Imagine Source, Germany) attached to a computer, and freezing behavior was analyzed using video-tracking software (Ethovision XT, Noldus, Netherlands) with manual counting for the duration of tail rattling behaviors.

\section{Gustatory stimulus}

For fiber photometry, mice were placed in the same arena for auditory and visual stimulus experiments with an additional $2 \mathrm{~cm}$ drilled hole. The water bottle spout was inserted into the hole, and the calcium signal was measured when the mice were licking. The bottle was filled with water or quinine $(0.5 \mathrm{mM}$, QU109, Spectrum Chemical, USA). For the loss-of-function experiment, mice were water-deprived overnight. The next day, mice were placed in a homecage with a water-, and $0.5 \mathrm{mM}$ quinine-containing bottle inserted into the water valve slot. Mice were allowed to drink for $10 \mathrm{~min}$ without habituation. All the trials were recorded by a USB camera (DFK 33GX236, Imagine Source, Germany) attached to a computer, and the licking behaviors were counted manually.

\section{Olfactory stimulus}

For fiber photometry, mice were placed in the same arena for gustatory stimulus experiments. Water- or Trimethylthiazoline (TMT, 97\%, $5 \mu$ l, 1G-TMT-97, BioSRQ, USA)-soaked cotton swap was introduced into the hole. Calcium signals were measured when mice smelled the cotton swap. For the loss-of-function experiment, mice movement was tracked in a two-chamber arena $(30 \times 60 \times 30 \mathrm{~cm})$ with a USB camera (DFK 33GX236, Imagine Source, Germany) using video-tracking software (EthoVision XT 12, Noldus, Netherlands). Two Petri dishes with small holes were placed in each chamber (one at the corner of the left chamber, and the other to the corner of the right chamber). On day 1, mice were able to habituate and explore the arena for $10 \mathrm{~min}$. The next day, a water-soaked cotton swap, or TMT-soaked cotton swap were placed in each dish. Mice were first placed at the center and monitored for 10 minutes as they interreacted with the two dishes.

\section{Foot shock}

1214

A fear-conditioning chamber (26 × $30 \times 33 \mathrm{~cm}$, ENV-007CT, MED Associates INC, USA) with a metal grid floor (ENV-005, MED Associates INC, USA) connected to a standalone aversive electric shock stimulator (ENV-414S, MED Associates INC, USA) was used for foot shock delivery. A USB camera (DFK 33GX236, Imagine Source, Germany) was connected to a computer, and the video tracking software (Ethovision XT, Noldus, Netherlands) was used for shock delivery and behavioral analysis. The chamber was enclosed in a light- and sound-attenuating cubicle (ENV-018MD, MED Associates INC, USA). The chamber was cleaned with $70 \%$ ethanol and double distilled water between each trial.

For fiber photometry and the loss-of-function experiment, mice were placed inside the chamber without habituation. After $2 \mathrm{~min}$ of baseline, an electric shock ( $2 \mathrm{~s}, 0.6 \mathrm{~mA})$ was delivered, and the behavior was recorded for an extra 2 min. Freezing behavior was monitored before (habituation), after (conditioning), and one day after (post-test) the shock.

\section{Elevated plus maze test}

A custom-built elevated plus maze with two transparent closed arms $(77 \times 7 \times 30 \mathrm{~cm})$ and two open arms $(77 \times 7 \times 2 \mathrm{~cm}$ ) was used to monitor the anxiety-like behaviors of test mice. This maze was elevated $70 \mathrm{~cm}$ above ground for all tests. Mice were placed to the tip of the open arm by facing towards the center of the maze. The behavior was video recorded for $10 \mathrm{~min}$ and tracked with a video-tracking software (EthoVision 
XT 12, Noldus, Netherlands). Both $70 \%$ ethanol solution and deionized water were used to clean the maze immediately after each trial. on a heated hot plate $\left(48\right.$ or $55^{\circ} \mathrm{C}, \mathrm{PE} 34$, IITC Life Science, USA). The latency of various pain responses (hind paw shake, lick, or jump) was measured manually.

A Dynamic Plantar Aesthesiometer (37450, Ugo Basile, Italy) was used to measure the mechanical pain thresholds. Mice were placed inside a Plexiglas chamber $(10 \times 10 \times 13 \mathrm{~cm})$ on a metal mesh floor and were habituated for $30 \mathrm{~min}$. The max force of the system was set to reach $50 \mathrm{~g}$ at $20 \mathrm{~s}$. The blunt metal rod of the aesthesiometer was placed under the hind paw and gradually protruded as the mice were immobile but awake. The latency and force delivered were automatically recorded as the mouse withdraw hind paw from the metal rod. The measurement was performed 5 times with 5-10 min intervals in between trials and averaged for a final mechanical threshold value.

\section{Optogenetics}

A $470 \mathrm{~nm}$ laser (LRD-0470-PFFD-00100-05, LaserGlow Tech., Canada) was used for all optogenetic experiments in this study. Optic fibers were bilaterally connected to pre-implanted optic ferrules on the mice. All mice were optogenetically stimulated $90 \mathrm{~min}$ before sacrifice for cFos immunohistochemistry.

The experiments were performed as described in the 'Hot plate test' section above with minor modification for optogenetic stimulation. The order of laser ON or OFF was counterbalanced, and the interval time between each experiment was more than $30 \mathrm{~min}$.

The experiments were performed as described above in the 'Electronic von Frey test' with minor modification for optogenetic stimulation. The laser was on immediately before the metal rod touched the paw pad and turned off right after paw withdrawal. camera (DFK 33GX236, Imagine Source, Germany) using video-tracking software (EthoVision XT 12, Noldus, Netherlands). After connecting the optic fiber, mice were placed in one side of the chamber. No stimulation was given for 10 min baseline. Afterward, one side of the chamber was randomly selected, and the mouse was photostimulated $(20 \mathrm{~Hz}$ for cell body stimulation, and $40 \mathrm{~Hz}$ for terminal stimulation, 8-9 $\mathrm{mW}$ ) for $20 \mathrm{~min}$. The stimulated side was counterbalanced between animals. Mice showing over 15\% preference to one side during baseline were excluded.

\section{Context-dependent optogenetic conditioning}

An open field arena $(40$ x 40 x $30 \mathrm{~cm}$ ) was used for context-dependent threat conditioning. After 10-min habituation to head-attached optic fibers in the home cage, mice were placed in the novel open field area and received photostimulation $(20 \mathrm{~Hz}$, and $8-9 \mathrm{~mW})$ throughout the experiment. After $24 \mathrm{~h}$, the mouse was re-introduced in the same context to test whether the photo-stimulation produced aversive memory. All the trials were recorded by a USB camera (DFK 33GX236, Imagine Source, Germany) attached to a computer, and freezing behavior was analyzed by a video-tracking software (EthoVision XT 12, Noldus, Netherlands). Auditory cue dependent optogenetic conditioning 
The same fear-conditioning chamber and the settings as described in the 'Foot shock' section above were

1273

1274

1275

1276

1277

1278

1279

1280

1281

1282

1283

1284

1285

1286

1287

1288

1289

1290

1291

1292

1293

1294

1295

1296

1297

1298

1299

1300

1301

1302

1303

1304

1305

1306

1307

1308

1309

1310

1311

1312

1313

1314

1315 used. Two speakers (AX210, Dell, USA) were placed beside the chamber for CS. On day 1, the test mouse was habituated with the conditioning chamber, which was cleaned with $70 \%$ ethanol and DW immediately after each test. During habituation, optic fibers were connected bilaterally to the optic ferrules on the mouse's head, and the CS+ $(30 \mathrm{~s}, 3 \mathrm{kHz}$ pure tone, $75 \mathrm{~dB})$ was delivered to the test mouse six times with random inter-event intervals. On day 2 , the test mouse was returned to the same context with optic fibers connected and received 10-s photostimulation $(20 \mathrm{~Hz}$ frequency for cell body and $40 \mathrm{~Hz}$ for terminal stimulation, 8-9 $\mathrm{mW}$ intensity) as the US, which was co-terminated with CS+ six times with random interevent intervals. On day 3 , the conditioned mouse without the optic fiber connected was returned to the same context for $2 \mathrm{~min}$. On day 4, a conditioned mouse without the optic fiber connected was introduced to a new context (a glass cylinder wrapped with a non-transparent material), and the CS+ was delivered without the US. All the trials were recorded by a USB camera (DFK 33GX236, Imagine Source, Germany) attached to the computer, and freezing behavior was analyzed by a video-tracking software (EthoVision XT 12, Noldus, Netherlands).

\section{Immunohistochemistry}

Mice were perfused intracardially with \% PFA solution in PBS, and the brain was extracted. The brain was kept in 4\% PFA overnight for post-fixation and dehydrated in $30 \%$ sucrose for 1-2 days before sectioning. Frozen brains were cut into $40 \mu \mathrm{m}$ coronal slices with a cryostat (CM 1950, Leica, USA) and washed with PBST (Phosphate buffered saline with 0.1\% Tween-20 (BP337-500, Fisher BioReagents, USA)). Initial blocking was performed by $1 \mathrm{hr}$ incubation with 3\% normal donkey serum (NDS, 017-000-121, Jackson ImmunoResearch Laboratories, Inc., USA). After another round of washing with PBST, the slices were incubated with anti-GFP (diluted 1:100 in 3\% NDS, GFP-1020, Aves, USA), anti-fos (1:10000, rabbit polyclonal), anti-Nav1.7 (1:200, ASC-008, Alomone), anti-Cav2.1 (1:100, ACC-001, Alomone, Isreal), or anti-FAAH $\left(1: 250,101600\right.$, Cayman, USA) antibody at $4{ }^{\circ} \mathrm{C}$ overnight. The next day, brain tissues were rinsed with PBST, then incubated with anti-rabbit Alexa Fluor ${ }^{\circledR}$ 647-secondary antibody (1:500, 711-605152, Jackson ImmunoResearch Laboratories, Inc., USA), and / or anti-chicken Alexa Fluor ${ }^{\circledR} 488$-secondary antibody (1:500, Jackson ImmunoResearch Laboratories) for $1 \mathrm{~h}$. After washing these brain slices with PBS, they were mounted on slide glass (12-550-143, Fisher Scientific, USA) with DAPI containing mounting solution.

\section{Preparation of acute brain slices and electrophysiology}

Mice were anesthetized with isoflurane and perfused via the vascular system using ice-cold cutting solution (110.0 mM choline chloride, $25.0 \mathrm{mM} \mathrm{NaHCO}_{3}, 1.25 \mathrm{mM} \mathrm{NaH}_{2} \mathrm{PO}_{4}, 2.5 \mathrm{mM} \mathrm{KCl}, 0.5 \mathrm{mM} \mathrm{CaCl}_{2}, 7.0 \mathrm{mM}$ $\mathrm{MgCl}_{2}, 25.0 \mathrm{mM}$ glucose, $5.0 \mathrm{mM}$ ascorbic acid and $3.0 \mathrm{mM}$ pyruvic acid, bubbled with $95 \% \mathrm{O}_{2}$ and $\%$ $\mathrm{CO}_{2}$ ). After decapitation, brains were quickly removed and chilled in an ice-cold cutting solution. Coronal slices containing the SPFp, PBel $(250 \mu \mathrm{m})$ or the amygdaloid complex $(300 \mu \mathrm{m})$ were cut by using a Leica VT 1200S Vibratome (Leica Biosystems Inc.), and subsequently transferred to a storage chamber containing artificial cerebrospinal fluid (aCSF; $124 \mathrm{mM} \mathrm{NaCl}, 2.5 \mathrm{mM} \mathrm{KCl}, 26.2 \mathrm{mM} \mathrm{NaHCO}, 1.2 \mathrm{mM}$ $\mathrm{NaH}_{2} \mathrm{PO}_{4}, 13 \mathrm{mM}$ glucose, $2 \mathrm{mM} \mathrm{MgSO} 4$ and $2 \mathrm{mM} \mathrm{CaCl}_{2}$, at $32{ }^{\circ} \mathrm{C}, \mathrm{pH} 7.4$, bubbled with $95 \% \mathrm{O}_{2}$ and $5 \%$ $\left.\mathrm{CO}_{2}\right)$. After at least $30 \mathrm{~min}$ recovery time, slices were transferred to room temperature $\left(22-24^{\circ} \mathrm{C}\right)$ for at least $60 \mathrm{~min}$ before use. Slices were transferred into the recording chamber, perfused with aCSF (flow rate around $2 \mathrm{ml} / \mathrm{min}$ ). The temperature of aACSF was held constant at $32^{\circ} \mathrm{C}$ by $\mathrm{TC}-324 \mathrm{C}$ temperature controller (Warner Instruments). Since CGRP-positive neurons express EGFP under the Calca promoter, 
they were visualized under Scientifica Microscope equipped with epifluorescence illumination at $490 \mathrm{~nm}$ LED. The Astr, LA, and IC neurons were visualized under trans-illumination. Whole-Cell patch clamp was performed with Multiclamp 700B amplifiers (Molecular Devices). Signals were digitized at $10 \mathrm{kHz}$ with Digidata 1550B (Molecular Devices). For evoked EPSCs, synaptic responses were evoked with a broken glass pipette positioned $100 \mu \mathrm{m}$ away from the recording glass electrode (3.0 5.0 MOm, back filled with internal solution: $\mathrm{CsMeSO}_{3} 130 \mathrm{mM}, \mathrm{CsCl}$ 5, HEPES $10 \mathrm{mM}, \mathrm{MgCl}_{2} 2.5 \mathrm{mM}$, EGTA $0.6 \mathrm{mM}$, Sodium phosphocreatine $10 \mathrm{mM}, \mathrm{Na}_{2} \mathrm{ATP} 4 \mathrm{mM}$ and $\mathrm{Na}_{3} \mathrm{GTP} 0.4 \mathrm{mM}, \mathrm{pH}$ 7.23, $285 \mathrm{Osm}$ ). The stimulus was given at $0.1 \mathrm{~Hz}$. AMPA EPSC was recorded holding at $-70 \mathrm{mV}$ for 10 to 30 sweeps to get a stable response. NMDA EPSC was recorded at $+40 \mathrm{mV}$ for $10-15$ sweeps. To ensure that the EPSCs were stable, the holding potential was set to $-70 \mathrm{mv}$ to check the AMPA EPSC change after NMDA EPSC recording. Evoked EPSCs were recorded with picrotoxin $(100 \mu \mathrm{M})$ in the aCSF. mEPSCs were recorded in the presence of tetrodotoxin $(1 \mu \mathrm{M})$ and picrotoxin $(100 \mu \mathrm{M})$. To record optogenetically evoked EPSC and IPSC, slices were harvested from the AAV-DIO-ChR2-EYFP injected Calca ${ }^{\text {Cre }}$ mice brain. 2-ms $470 \mathrm{~nm}$ LED light (TTL from Clampex to Cool Led pE-300) was illuminated through 40X NA 0.8 objective lens at $0.1 \mathrm{~Hz}$ to evoke optogenetically evoked postsynaptic current. The internal solution was calculated to make chloride reversal potential at $-70 \mathrm{mV}$. EPSCs were recorded at $-70 \mathrm{mV}$, and IPSCs were recorded at $0 \mathrm{mV}$. CNQX $(10 \mu \mathrm{M})$ was perfused to check the glutamatergic synapse. EPSCs and IPSCs were analyzed using pCLAMP 10 software (Molecular Devices). NMDA EPSCs were defined as signals 100 ms apart from stimulus artifacts. mEPSCs were analyzed using Mini Analysis Program (Synaptosoft).

\section{Imaging}

The images were taken with an automatic fluorescence microscope (BZ-X710, Keyence, USA) using included imaging software (BZ-X viewer, Keyence, USA) or with a scanning confocal microscope (FV 1000, Olympus, Japan) using with Fluoview software (Olympus, Japan). For quantification purposes, images were processed with the same gain, offset, and exposure time. Cell counting for retrograde tracing was done manually.

\section{RiboTag Transcriptomic Profiling}

To label the active transcriptome of CGRP ${ }^{\text {SPFp }}$ and CGRP ${ }^{\text {PBel }}$ neurons, we crossed Calca $^{\text {CreER }}$ with RiboTag $R p l 22^{H A / H A}$ mice. To induce gene expression, $200 \mu \mathrm{l}$ of tamoxifen freshly prepared with $20 \mathrm{mg} / \mathrm{ml} \mathrm{in}$ corn oil and dissolved overnight with continuous agitation was administered intraperitoneally for five consecutive days in each mouse. Experiments were performed two weeks after the final tamoxifen injection. $250 \mu \mathrm{m}$ thick slices containing the PBel and the SPFp were obtained using a VT 1200S Vibratome (Leica, Germany). The region of interest was further dissected using surgical scissors under the stereoscope. Tissues of interest from four Calca ${ }^{\text {CreER}}$; RiboTag crossed mice (10-12 weeks old) were collected into 1.5 $\mathrm{mL}$ microcentrifuge tubes containing homogenization buffer and were mechanically dissociated and lysed using pellet pestles (Cat.no.7495211500-DS, DWK Life Sciences LLC, USA). Total RNA was extracted from $15 \%$ of cleared lysate for input samples. The remaining lysate was incubated with mouse anti-HA antibody (Cat.no.MMS-101R, Biolegend, USA) and was rocked for 4 hours at $4{ }^{\circ} \mathrm{C}$. Afterward, magnetic beads (Cat.no.88803, Thermo Fisher Scientific, USA) were added, and the solution was incubated overnight, rocking at $4{ }^{\circ} \mathrm{C}$. The beads were washed three times in high salt solution. The bound ribosomes and RNA were separated from the beads by $30 \mathrm{~s}$ of vortexing in RLT lysis buffer as IP. All RNA samples were purified from the IP and corresponding input samples (Qiagen RNeasy Mini Kit, cat.no. 74104), then quantified with the Qubit RNA Assay Kit (Invitrogen, USA) and analyzed with the RNA 6000 Pico Kit 
(Agilent, USA). Isolated RNA was prepared using the Trio RNA-Seq (Cat. No. 0507-08; NuGEN, USA). Briefly, cDNA was synthesized from the total RNA using reverse transcriptase with oligo dT and resynthesized to produce double-stranded cDNA. After amplifying double-stranded cDNA, cDNA was purified with AMPure XP Beads (Cat. No. A63881; Beckman Coulter, USA), fragmented to the library, and classified using a barcoded adaptor. All libraries were quantified by qPCR and analyzed with the RNA 6000 Pico Kit. RNA library quality was checked using the 2100 Bioanalyzer (Agilent, USA). Barcoded samples were pooled and sequenced on the NextSeq500 (Illumina, USA) with the 75 bp read length singleend. Image analysis and base calling were conducted using the Illumina CASAVA-1.8.2 software. The FastQC package was utilized to evaluate the sequencing read quality. Fastq reads were then aligned to the reference genome (GRCm38.p6) using the STAR tool (version 2.7.2) in a pair-end mode. The quantification package RSEM (version 1.2.28) was employed to calculate gene expression from BAM files using the default setting changed to pair-end mode. In doing so, estimated count and TPM (Transcripts Per Million) were generated. Fold changes were calculated from TPM values (estimated counts, > 20) between HA-tag and HA negative controls., The ggplot 2 package from $R$ was utilized to visualize fold changes. UP (> 2.5fold change) and DOWN (<-2.5-fold change) were highlighted with orange and blue colors, respectively.

\section{Statistical analysis}

1377 All data are shown as mean \pm s.e.m. and analyzed using Student's t-test, one-way ANOVA with Tukey's post hoc comparison, and two-way ANOVA with Sidak's post hoc comparison. All the statistical analyses were done using Prism 6 (GraphPad Software Inc., USA). NS p $>0.05, * \mathrm{p}<0.05, * * \mathrm{p}<0.01$, *** $\mathrm{p}<$ 0.001 


\section{Supplementary Figure Legends}

A

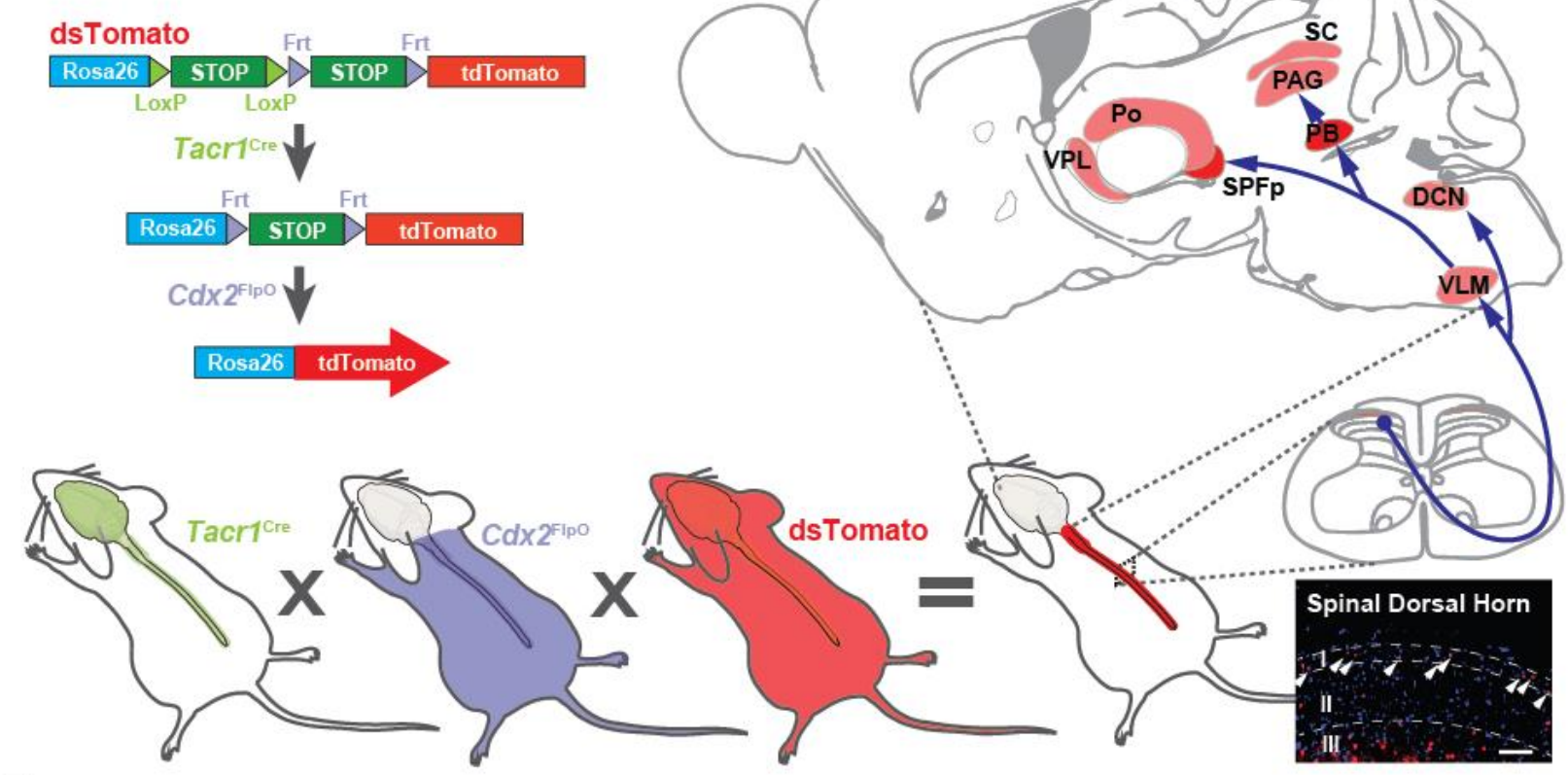

B
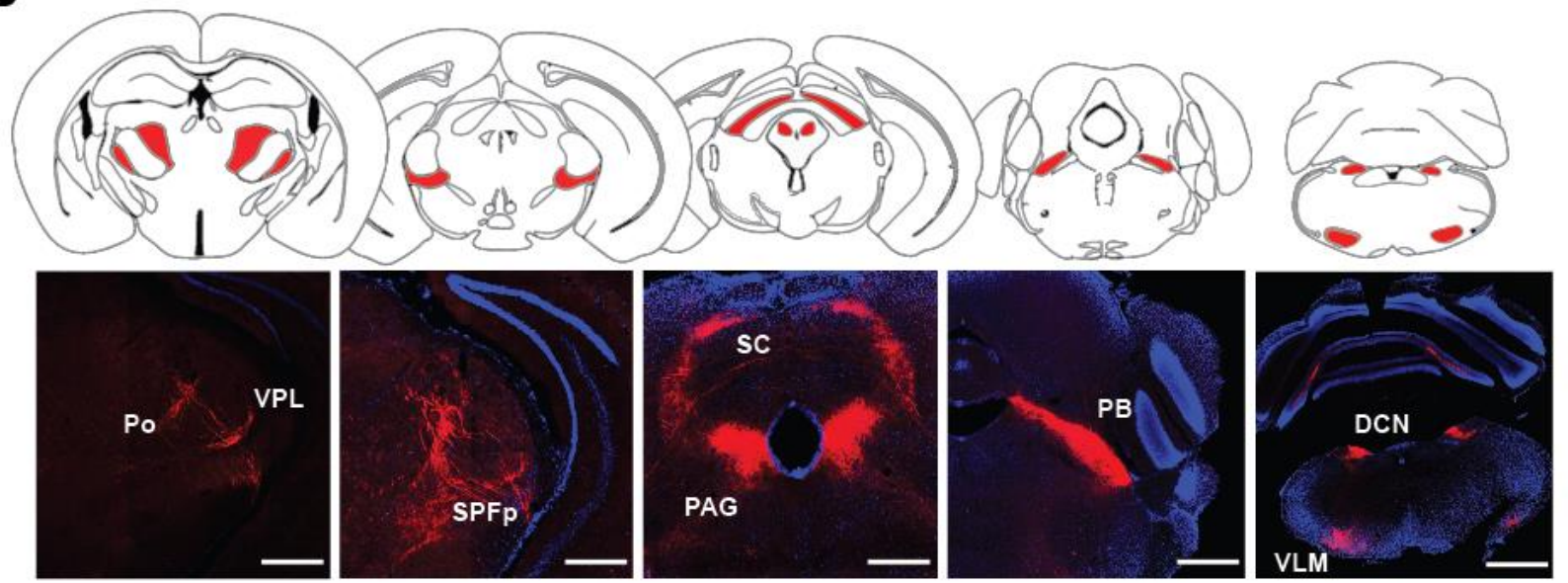

Figure S1. Identification of direct spino-recipient areas in the brain by specific genetic labeling of Tacrl-expressing spinal projection neurons.

(A) Schematics of the triple cross strategy to specifically label Tacrl-expressing neurons in the spinal dorsal horn. Scale bar indicates $200 \mu \mathrm{m}$.

(B) Spinal Tacrl-expressing neurons send projections to the posterior complex of the thalamus (PO), ventral posterolateral nucleus of the thalamus (VPL), the ventral posteromedial nucleus of the thalamus (VPM), SPFp, superior colliculus (SC), periductal gray (PAG), PB, dorsal column nuclei (DCN) and 
A
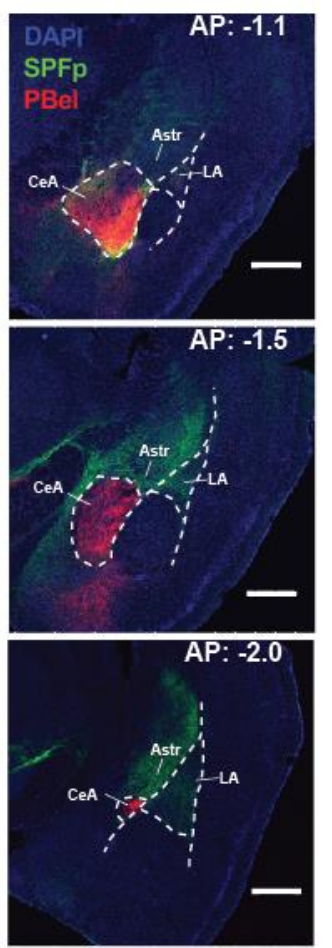

B
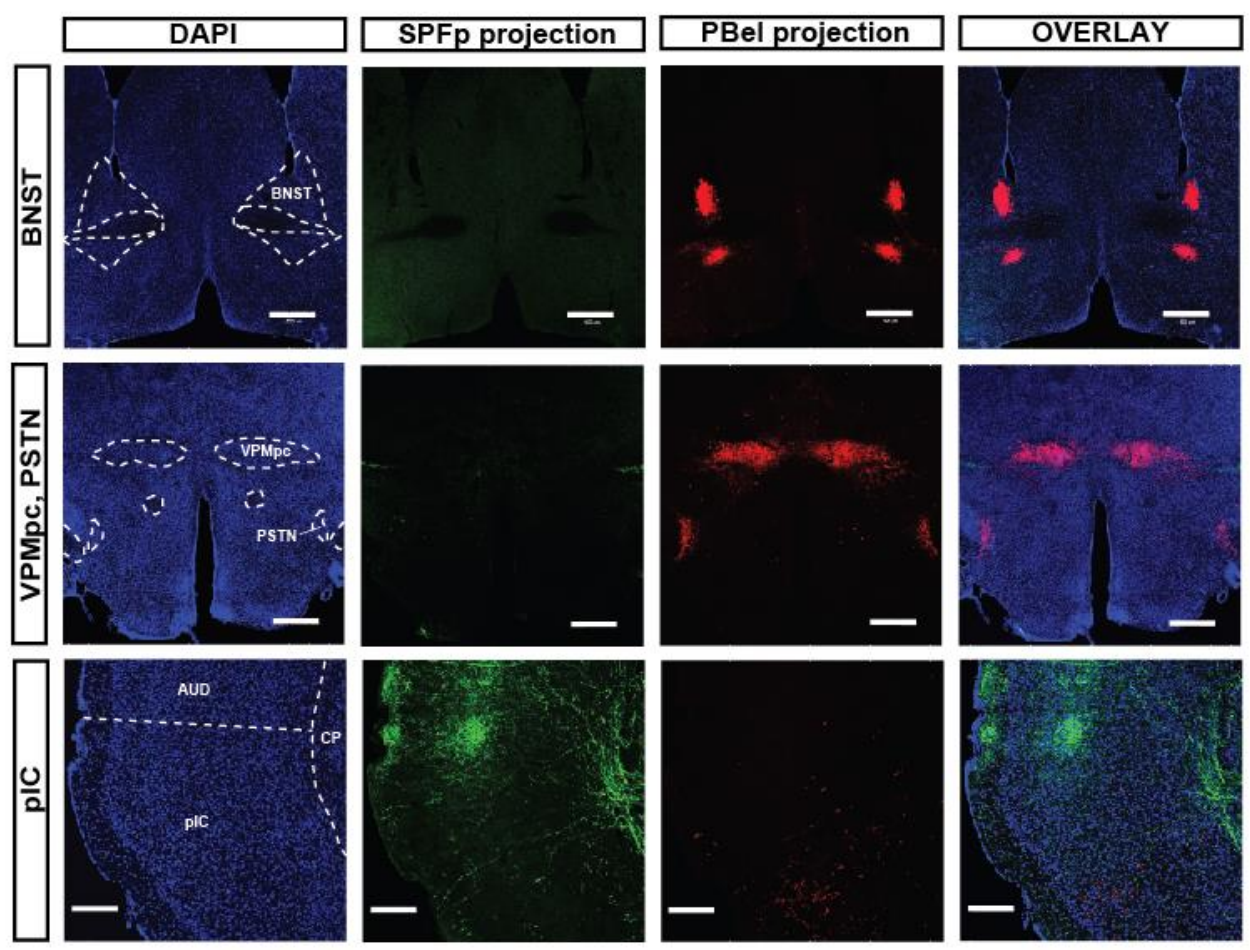

\section{Figure S2. Projections from CGRP ${ }^{\mathrm{SPFp}}$ and CGRP ${ }^{\mathrm{PBel}}$ neurons.}

(A) Additional images from Figure 1A, B. Projections are prominent in the amygdala regions and have distinct patterns along the anterior-posterior axis; AP: -1.1, -1.5 and -2.0 $\mathrm{mm}$ from bregma. Scale bar indicates $500 \mu \mathrm{m}$.

(B) The CGRP ${ }^{\mathrm{PBel}}$ neurons also project to BNST, VPMpc, PSTN, and ventral pIC. The CGRP ${ }^{\mathrm{SPF}}$ neurons project to the auditory cortex, and dorsal pIC. Scale bars indicate $500 \mu \mathrm{m}$ for BNST, and VPMpc; $200 \mu \mathrm{m}$ for $\mathrm{pIC}$. 

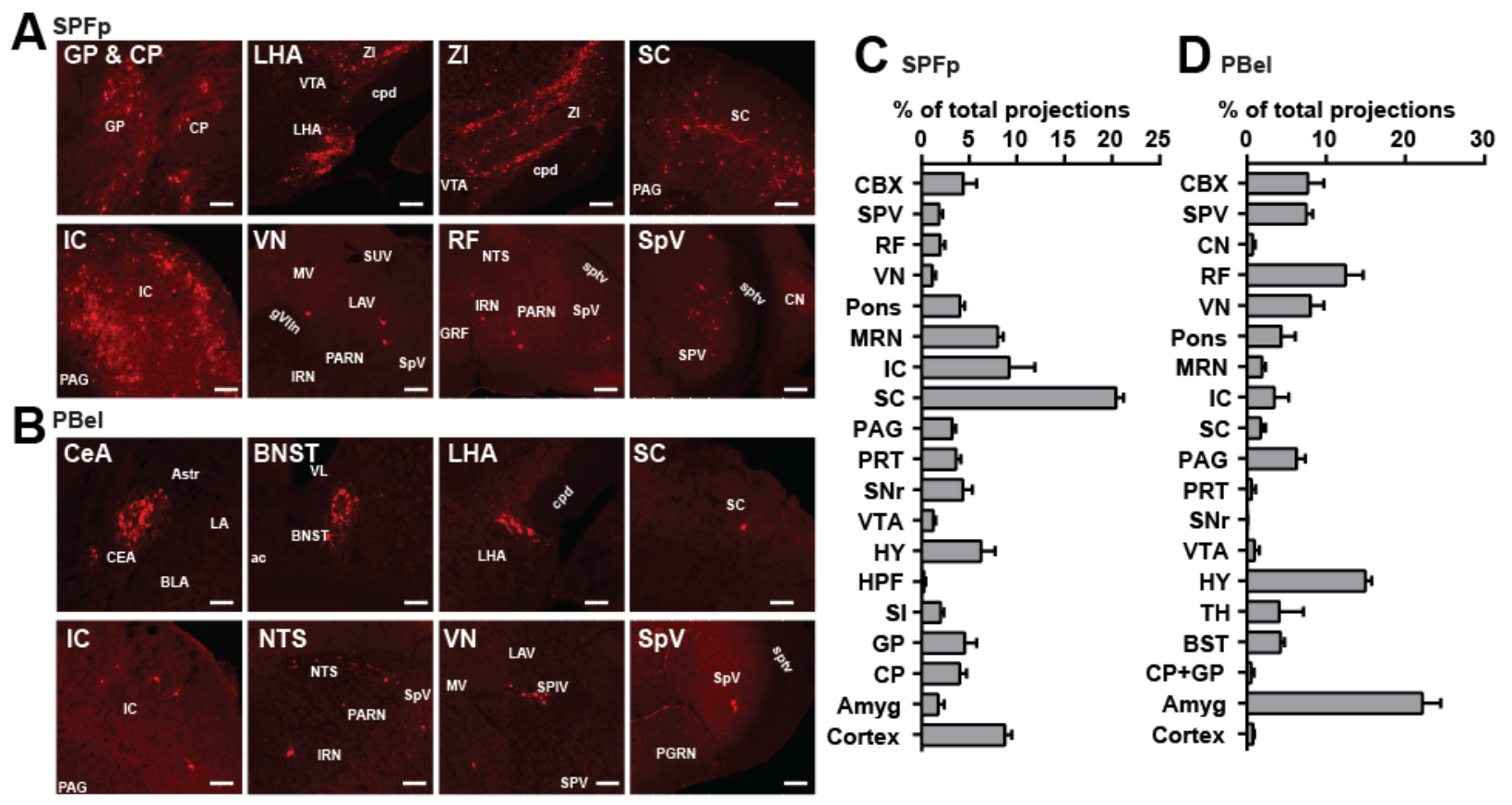

$E_{\text {sPFp }}$

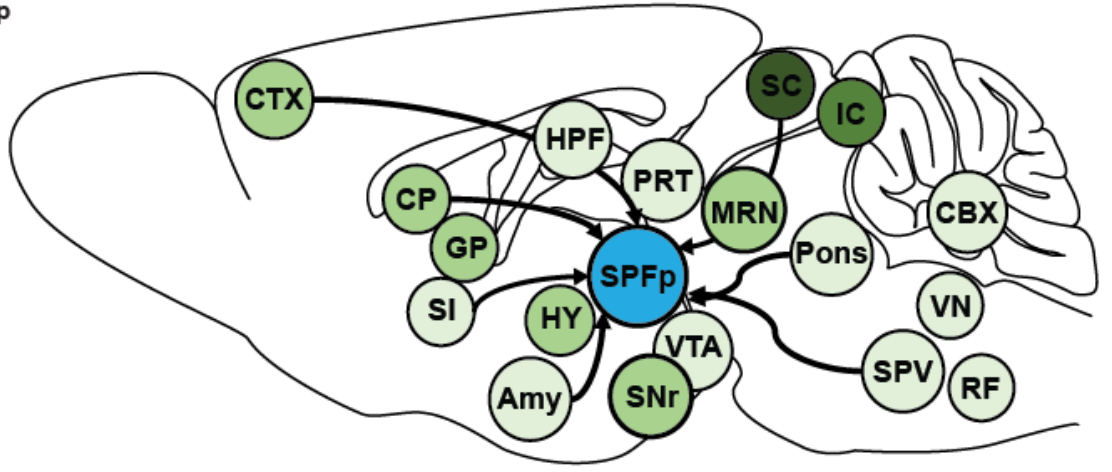

$\%$ projections

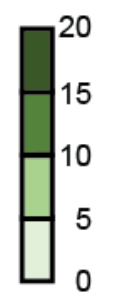

$\mathbf{F}_{\text {PBel }}$

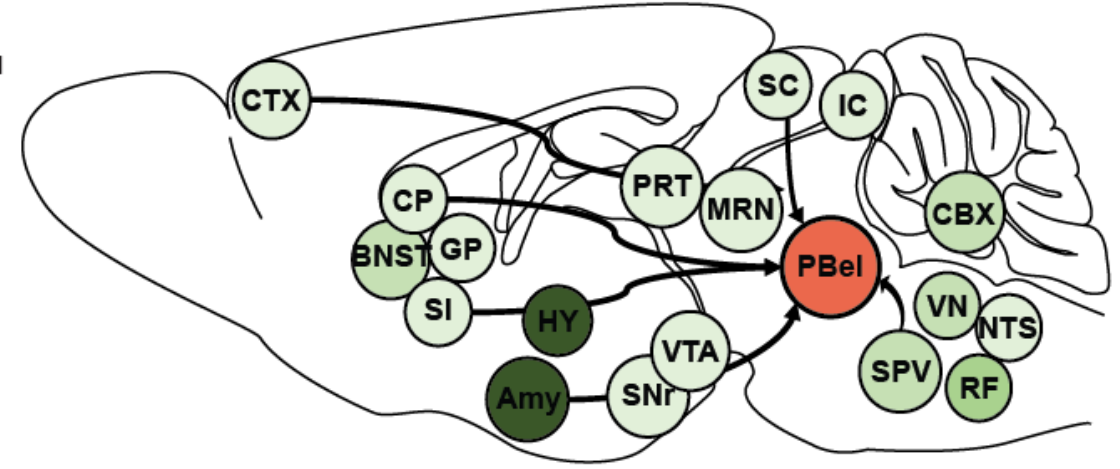

$\%$ projections

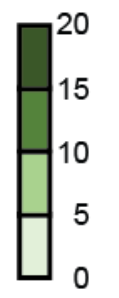

Figure S3. Retrograde tracing from CGRP ${ }^{\mathrm{SPF}}$ and CGRP ${ }^{\mathrm{PBel}}$ neurons.

1404 (A and B) Example schematic of brain regions that send inputs to CGRP ${ }^{\mathrm{SPF}}(\mathbf{A})$ and $\mathrm{CGRP}^{\mathrm{PBel}}$ neurons $(\mathbf{B})$.

1405 Scale bars indicate $100 \mu \mathrm{m}$.

1406 (C) Percentage of total projections from brain regions to CGRPSPFp neurons.

1407 (D) Percentage of total projections from brain regions to $\mathrm{CGRP}^{\mathrm{PBel}}$ neurons.

1408 (E and $\mathbf{F})$ Diagram of the projection (\%) from other brain regions to CGRP ${ }^{\text {SPFp }}(\mathbf{E})$ and CGRP ${ }^{\text {PBel }}$ neurons $1409(\mathbf{F})$. 
1410 CTX: cortex, Amy: amygdala, CP: striatum, GP: globus pallidus, BNST: bed nuclei of the stria terminalis, 1411 SI: substantia innominate, HPF: hippocampus, HY: hypothalamus, TH: thalamus, VTA: ventral tegmental 1412 area, SNr: substantia nigra, PRT: pretectal region, PAG: periaqueductal gray, SC: superior colliculus, IC: 1413 inferior colliculus, MRN: midbrain reticular nucleus, Pons: including the nucleus of the lateral lemniscus, 1414 pontine central gray, PBN, pontine reticular nucleus, VN: vestibular nucleus, NTS: nucleus tractus solitarius, 1415 SpV: trigeminal spinal nucleus, CBX: cerebellum. 

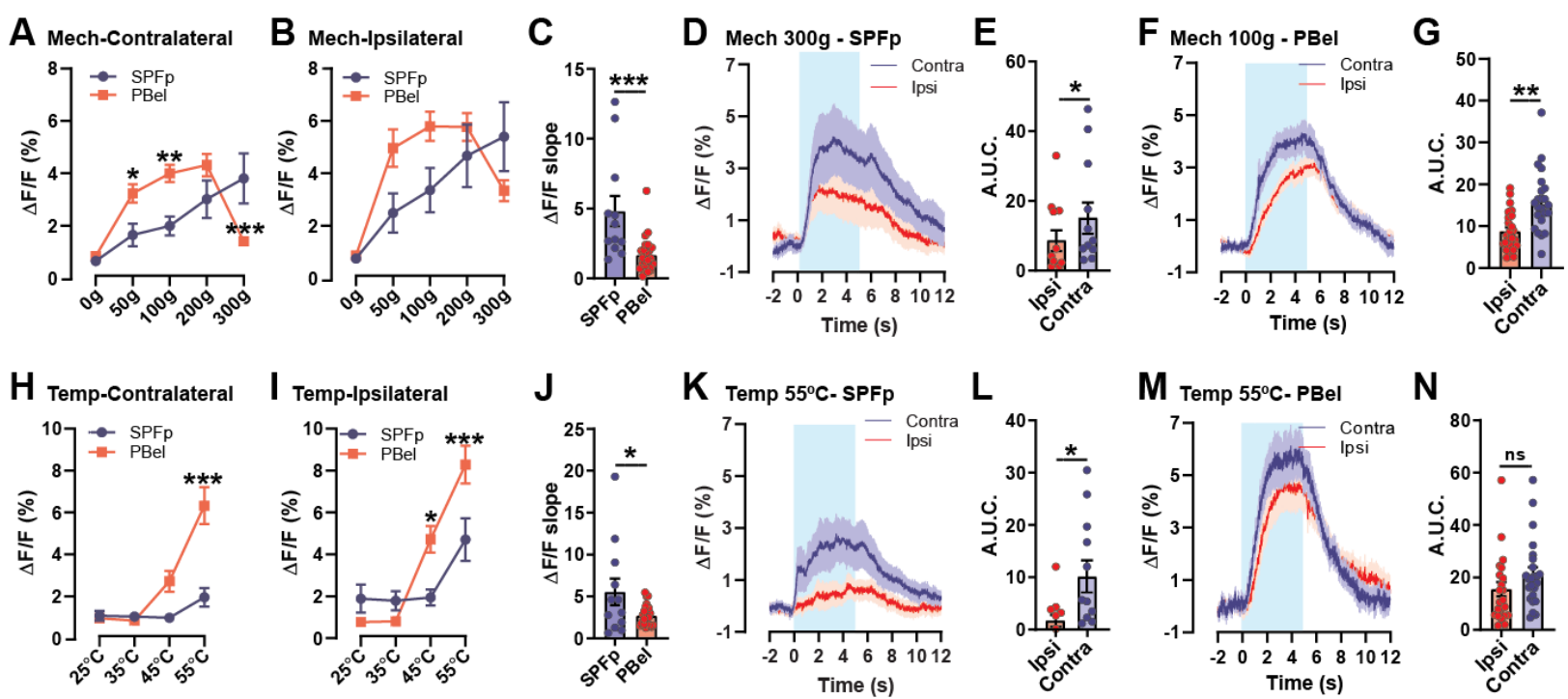

\section{Figure S4. CGRP ${ }^{\mathrm{SPF}}$ and CGRP ${ }^{\mathrm{PBel}}$ neurons are bilaterally stimulated by noxious stimuli.}

(A and B) Maximum calcium responses of the CGRP ${ }^{\text {SPFp }}$ and CGRP $^{\text {PBel }}$ neurons to ipsilateral (A) and contralateral (B) mechanical stimulation.

(C) CGRP ${ }^{\text {SPFp }}$ displayed faster increase of the calcium responses (bigger initial slope) to the contralateral mechanical stimulation (300 $\mathrm{g}$ for CGRP ${ }^{\mathrm{SPFp}}$ and $100 \mathrm{~g}$ for CGRP ${ }^{\mathrm{PBel}}$ neurons).

(D-G) Calcium responses of CGRP ${ }^{\text {SPFp }}$ (D and E) neurons by ipsilateral or contralateral $300 \mathrm{~g}$ stimulation and $\mathrm{CGRP}^{\mathrm{PBel}}$ ( $\mathbf{F}$ and $\mathbf{G}$ ) neurons by $100 \mathrm{~g}$ stimulation.

(H and I) Maximum calcium responses of the CGRP ${ }^{\text {SPFp }}$ and CGRP $^{\text {PBel }}$ neurons to ipsilateral (H) and contralateral (I) thermal stimulation.

(J) Comparison of the initial slope of both neurons to contralateral $55^{\circ} \mathrm{C}$ stimulation.

(K-N) Calcium responses of CGRP ${ }^{\mathrm{SPFp}}(\mathbf{K}$ and $\mathbf{L})$ neurons and $\mathrm{CGRP}^{\text {PBel }}(\mathbf{M}$ and $\mathbf{N})$ neurons by ipsilateral or contralateral $55^{\circ} \mathrm{C}$ stimulation.

\section{Statistics}

(A) Repeated measure two-way ANOVA showed significance in intensity $\mathrm{X}$ region interaction $(\mathrm{F}(4,136)$ $=11.80, \mathrm{p}<0.0001)$, intensity $(\mathrm{F}(4,136)=17.66, \mathrm{p}<0.0001)$, but not in region $(\mathrm{F}(1,34)=2.075, \mathrm{p}=$ 0.1589). SPFp and PBel were significantly different in $50(\mathrm{p}<0.05), 100(\mathrm{p}<0.01)$ and $300 \mathrm{~g}(\mathrm{p}<0.001)$ with Sidak's multiple comparisons test.

(B) Repeated measure two-way ANOVA showed significance in intensity $\mathrm{X}$ region interaction $(\mathrm{F}(4,136)$ $=5.468, \mathrm{p}=0.0004)$, intensity $(\mathrm{F}(4,136)=18.46, \mathrm{p}<0.0001)$, but not in region $(\mathrm{F}(1,34)=1.528, \mathrm{p}=$ $0.2249)$.

(C) SPFp: $4.82 \pm 1.08$ ( $\mathrm{n}=6$ mice, 12 trial), PBel: $1.68 \pm 0.26$ ( $\mathrm{n}=6$ mice, 24 trial). Unpaired $\mathrm{t}$ test (twotailed), $\mathrm{p}=0.0007$.

(E) SPFp; Ipsi: $8.51 \pm 3.00(\mathrm{n}=6$ mice, 12 trial), Contra: $15.06 \pm 4.51(\mathrm{n}=6$ mice, 12 trial). Paired t-test (two-tailed), $\mathrm{p}=0.0498$.

(G) PBel; Ipsi: $8.79 \pm 0.95$ ( $\mathrm{n}=6$ mice, 24 trial), Contra: $15.26 \pm 1.61$ ( $\mathrm{n}=6$ mice, 24 trial). Paired t-test (two-tailed), $\mathrm{p}=0.0012$. 
$1446(\mathrm{H})$ Repeated measure two-way ANOVA showed significance in intensity $\mathrm{X}$ region interaction $(\mathrm{F}(3,102)$ $1447=8.995, \mathrm{p}<0.0001)$, intensity $(\mathrm{F}(3,102)=17.79, \mathrm{p}<0.0001)$, and region $(\mathrm{F}(1,34)=11.19, \mathrm{p}=0.002)$.

1448 SPFp and PBel were significantly different in $55^{\circ} \mathrm{C}(\mathrm{p}<0.0001)$ with Sidak's multiple comparisons test.

1449 (I) Repeated measure two-way ANOVA showed significance in intensity $\mathrm{X}$ region interaction $(\mathrm{F}(3,102)=$ $145010.16, \mathrm{p}<0.0001)$, intensity $(\mathrm{F}(3,102)=40.99, \mathrm{p}<0.0001)$, but not in region $(\mathrm{F}(1,34)=2.885, \mathrm{p}=0.0986)$.

1451 SPFp and PBel were significantly different in $45(\mathrm{p}<0.05)$ and $55^{\circ} \mathrm{C}(\mathrm{p}<0.0001)$ with Sidak's multiple 1452 comparisons test.

1453 (J) SPFp: $5.55 \pm 1.59$ ( $\mathrm{n}=6$ mice, 12 trial), PBel: $2.72 \pm 0.26$ ( $\mathrm{n}=6$ mice, 24 trial). Unpaired t test (two1454 tailed), $\mathrm{p}=0.0210$.

1455 (L) SPFp; Ipsi: $1.74 \pm 1.12$ ( $\mathrm{n}=6$ mice, 12 trial), Contra: $10.15 \pm 3.08$ ( $\mathrm{n}=6$ mice, 12 trial). Paired t-test 1456 (two-tailed), $\mathrm{p}=0.0146$.

1457 (N) PBel; Ipsi: $15.55 \pm 2.71$ ( $n=6$ mice, 24 trial), Contra: $21.24 \pm 2.63$ ( $n=6$ mice, 24 trial). Paired t-test 1458 (two-tailed), $\mathrm{p}=0.1385$. 

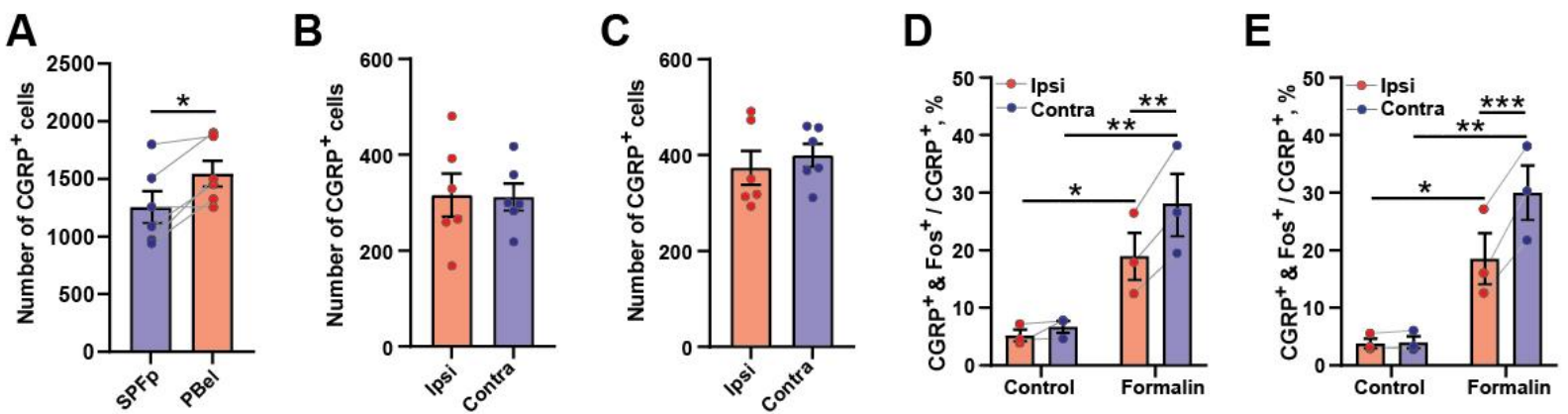

$\mathbf{F}$
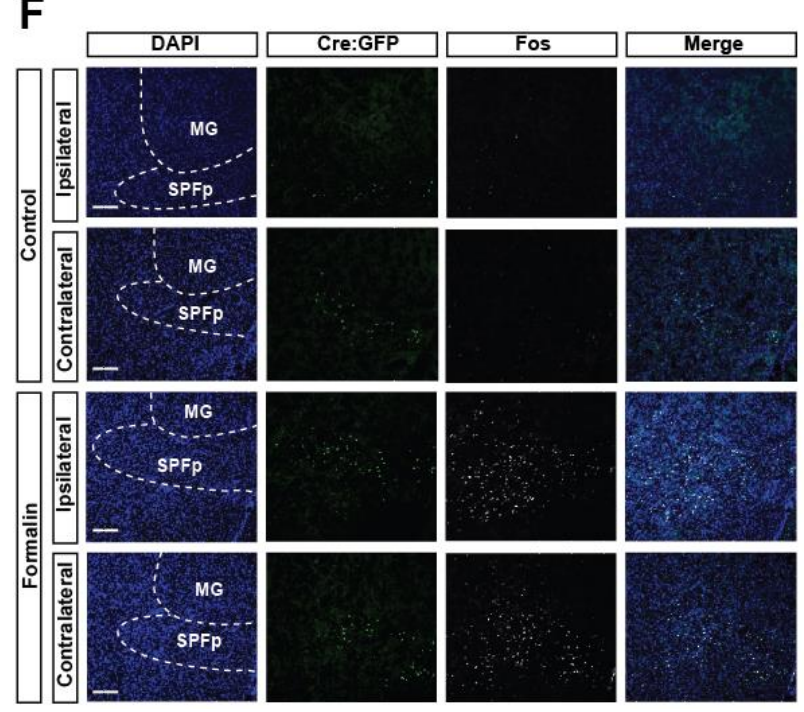

G
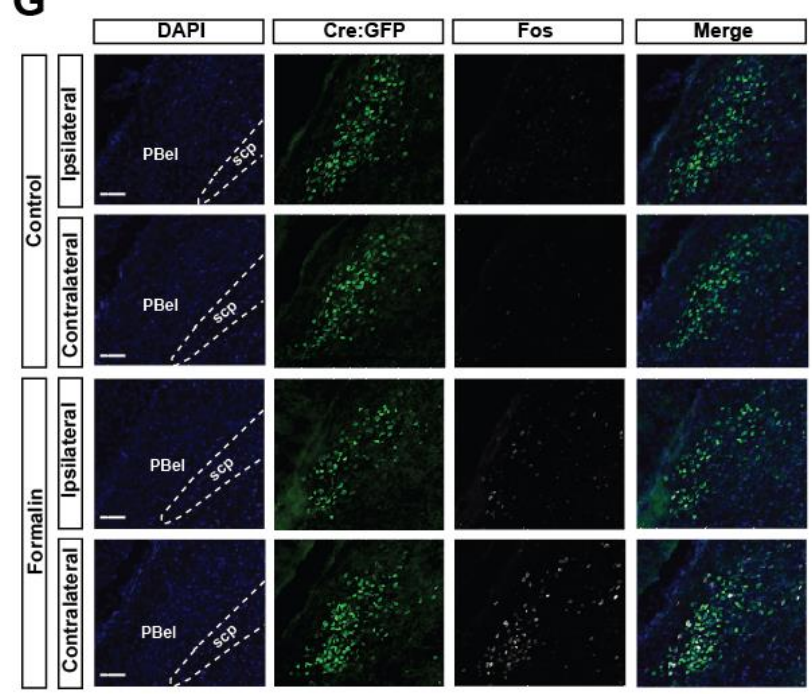

Figure S5. Bilateral activation of CGRPSPF and CGRP ${ }^{\mathrm{PBe}}$

neurons by unilateral formalin injection in the same animal.

1463 (A) Number of CGRP positive cells in the SPFp and PBel.

1464

\section{Statistics}
(A) SPFp: $1255 \pm 137.5$, PBel $1545 \pm 112.2$ ( $\mathrm{n}=6$ mice). Paired t-test (two-tailed), $\mathrm{p}=0.0188$.

1472

(B) SPFp; Ipsi: $315.5 \pm 44.94$, Contra: $311.8 \pm 27.85$ ( $\mathrm{n}=6$ mice). Paired t-test (two-tailed), $\mathrm{p}=0.9057$.

1473

(C) PBel; Ipsi: $373.0 \pm 35.34$, Contra $399.3 \pm 24.06$ ( $n=6$ mice). Paired t-test (two-tailed), $\mathrm{p}=0.2958$.

1474

(D) SPFp; Repeated measure two-way ANOVA showed significance in treatment $\mathrm{X}$ side interaction $(\mathrm{F}(1$,

1475 $4)=17.42, p=0.0140)$, treatment $(F(1,4)=13.28, p=0.0219)$, and side $(F(1,4)=33.97, p=0.0043)$.

1476 Ipsi vs contra was significant in formalin group $(\mathrm{p}<0.01)$. Control vs formalin was significantly different in both ipsi $(\mathrm{p}<0.05)$ and contra $(\mathrm{p}<0.01)$ with Sidak's multiple comparisons test. $4)=55.27, \mathrm{p}=0.0017)$, treatment $(\mathrm{F}(1,4)=19.57, \mathrm{p}=0.0115)$, and side $(\mathrm{F}(1,4)=58.66, \mathrm{p}=0.0016)$. 
A

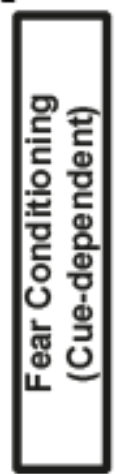

Day 1 (Habituation)

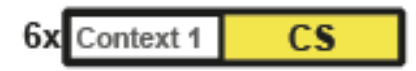

Day 2 (Conditioning)

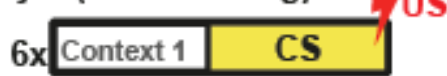

Day 3 (Cue test)

\begin{tabular}{|c|c|}
\hline Context 2 & CS \\
\hline
\end{tabular}

CS: $70 \mathrm{~dB}, 3 \mathrm{kHz}$ Tone
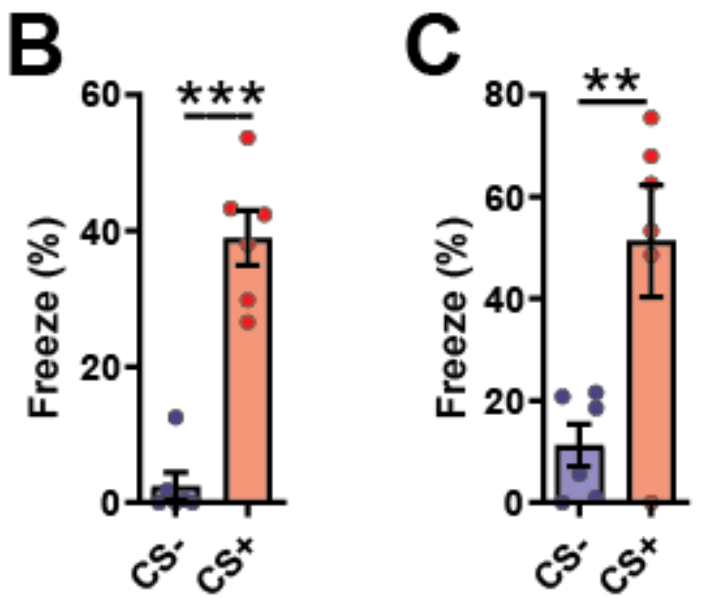

Figure S6. Cued fear conditioning with mice for CGRP ${ }^{\text {SPFp }}$ and CGRP ${ }^{\text {PBel }}$ fiber photometry.

(A) Behavioral scheme for cued fear conditioning. Low intensity $(70 \mathrm{~dB}, 3 \mathrm{kHz})$ tone was used as CS in order not to induce calcium activity by sound.

1486

$(\mathbf{B}$ and $\mathbf{C})$ Freezing was induced in both CGRP $\mathrm{SPFp}^{\mathrm{B}}(\mathbf{B})$ and $\mathrm{CGRP}^{\mathrm{PBel}}(\mathbf{C})$ group.

\section{Statistics}

1490

(B) SPFp; CS-: $2.47 \pm 2.04 \%$, CS+: $38.96 \pm 4.02 \%(\mathrm{n}=6)$. Paired t test (two-tailed), $\mathrm{p}<0.0001$.

1491

1492

(C) PBel; CS-: $11.27 \pm 4.14 \%, C S+51.31 \pm 11.00 \%(n=6)$. Paired t test (two-tailed), $\mathrm{p}=0.01$. 

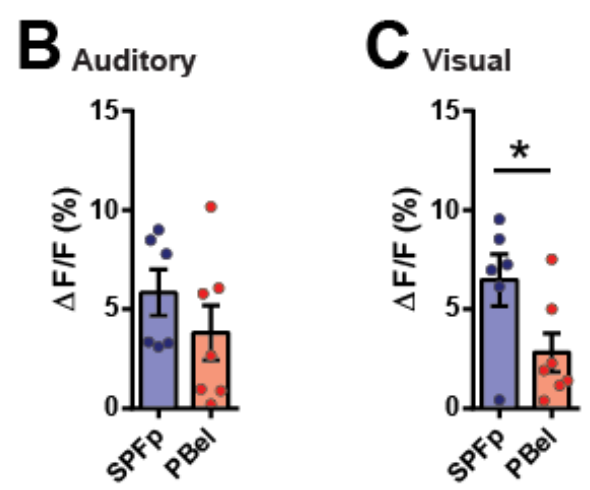

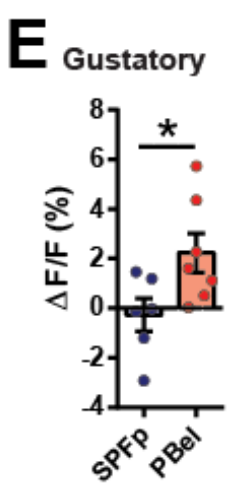

Figure S7. The CGRP ${ }^{\text {SPFp }}$ and CGRP ${ }^{\text {PBel }}$ neurons are differentially activated by multiple sensory threat cues.

(A-E) Calcium response (peak amplitude) in the CGRP ${ }^{\mathrm{SPF}}$ and $\mathrm{CGRP}^{\mathrm{PBel}}$ neurons by 2-s electric foot shock $(0.6 \mathrm{~mA})(\mathbf{A}), 85-\mathrm{dB}$ intense sound $(\mathbf{B})$, rapidly expanding looming disk $(\mathbf{C})$, TMT (D), and $0.5 \mathrm{mM}$ quinine solution $(\mathbf{E})$.

\section{Statistics}

(A) SPFp: $9.17 \pm 2.25 \%(\mathrm{n}=6)$, PBel: $5.70 \pm 1.03 \%(\mathrm{n}=6)$. Unpaired t test (two-tailed), $\mathrm{p}=0.1917$.

(B) SPFp: $5.84 \pm 1.17 \%(\mathrm{n}=6)$, PBel: $3.82 \pm 1.39 \%(\mathrm{n}=7)$. Unpaired t test (two-tailed), $\mathrm{p}=0.2987$.

(C) SPFp: $6.48 \pm 1.31 \%(\mathrm{n}=6)$, PBel: $2.81 \pm 0.96 \%(\mathrm{n}=7)$. Unpaired t test (two-tailed), $\mathrm{p}=0.0418$.

(D) SPFp: $-2.80 \pm 1.68 \%(\mathrm{n}=6)$, PBel: $2.68 \pm 1.07 \%(\mathrm{n}=6)$. Unpaired t test (two-tailed), $\mathrm{p}=0.0206$.

(E) SPFp: $-0.27 \pm 0.66 \%(\mathrm{n}=6)$, PBel: $2.24 \pm 0.79 \%(\mathrm{n}=7)$. Unpaired t test (two-tailed), $\mathrm{p}=0.0363$. 


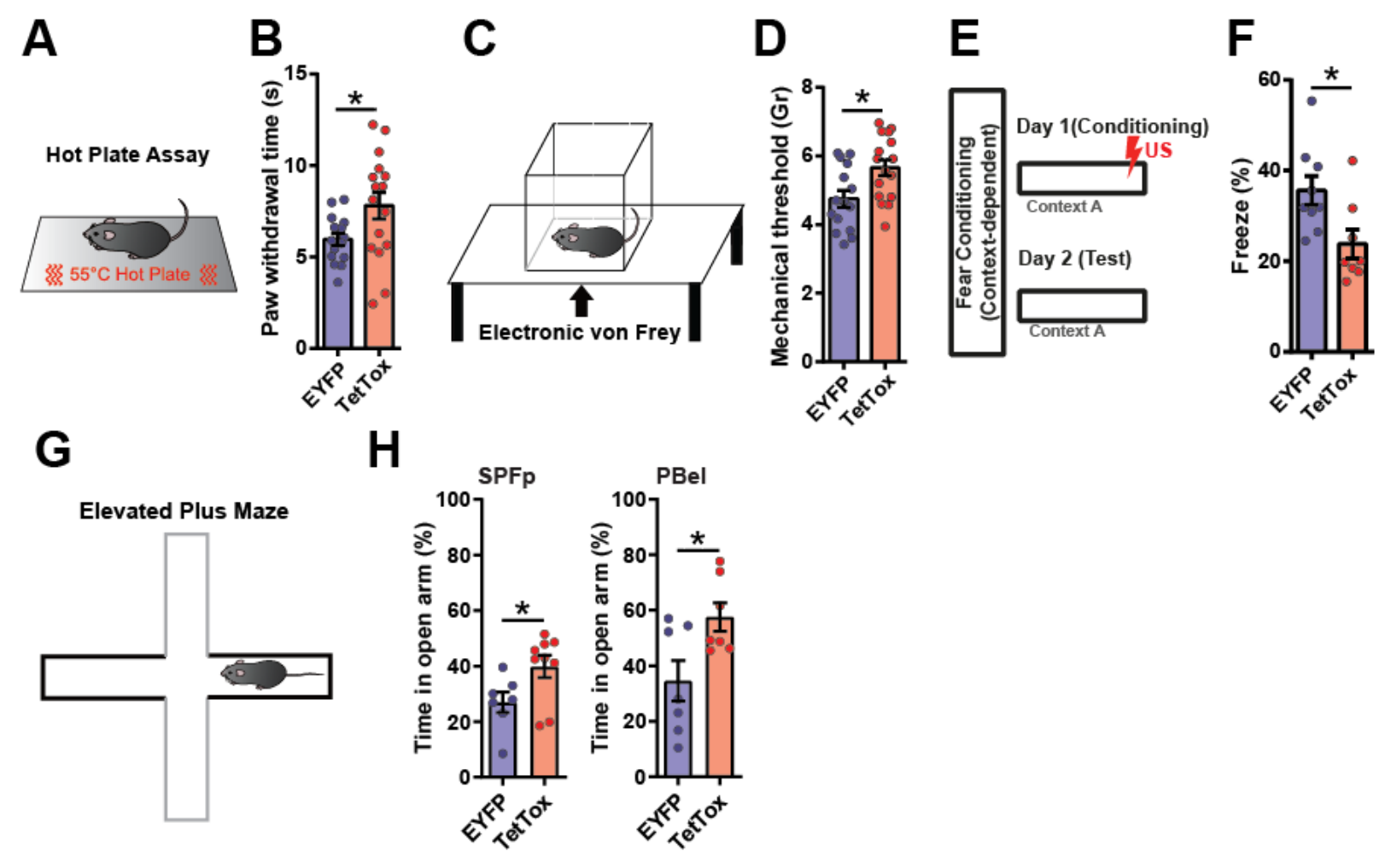

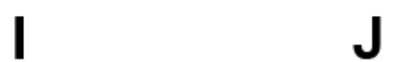

Hot Plate Assay

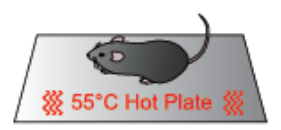

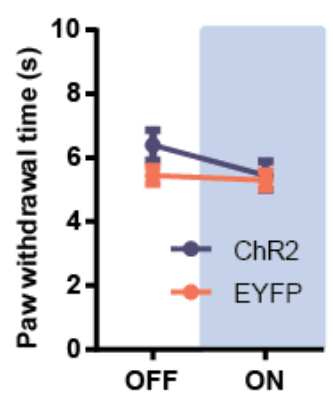

K
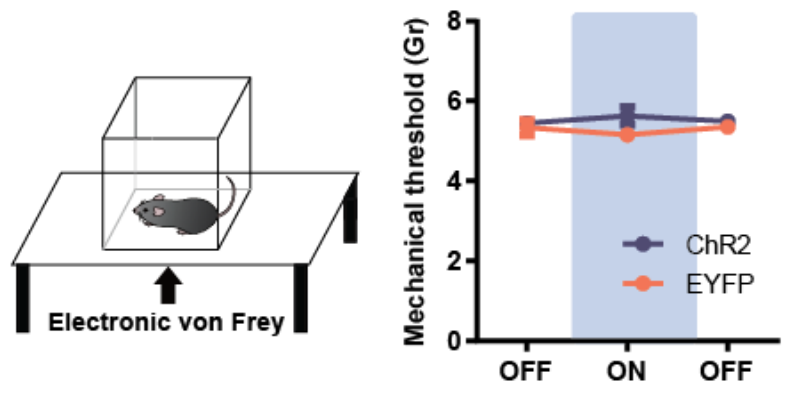

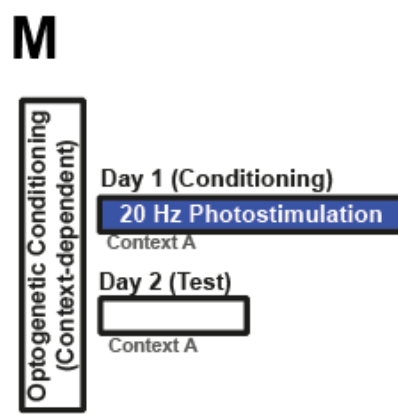

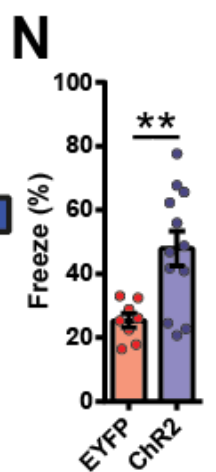

0

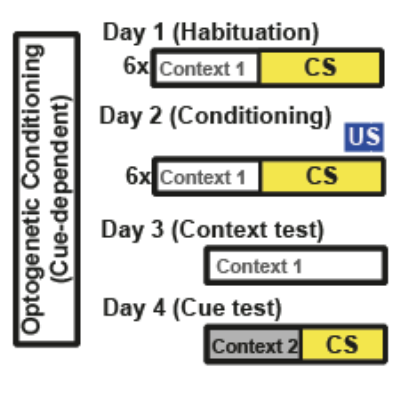

$\mathbf{P}$

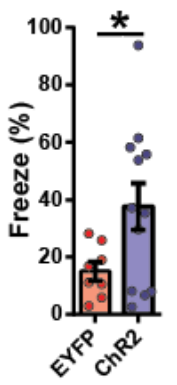

Q

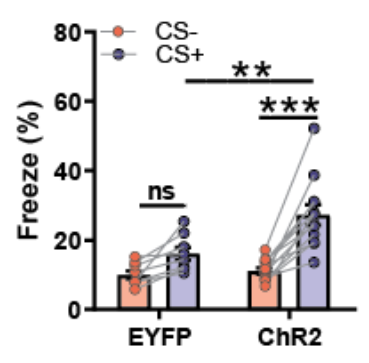

Figure S8. Manipulation of CGRP ${ }^{\mathrm{SPF}}$ neurons in behavior assays.

(A, and B) Hot plate assay $\left(55^{\circ} \mathrm{C}\right)$ with CGRP ${ }^{\mathrm{SPF}}$ silenced mice.

1510

(C, and D) Automatic von Frey assay with CGRP ${ }^{\mathrm{SPF} p}$ silenced mice.

1511

(E) Experimental design for contextual fear conditioning. 
1512 (F) Quantification of freezing $24 \mathrm{hr}$ after contextual fear conditioning.

1513 (G) Schematic diagram of the elevated plus maze (EPM) test.

1514 (H) The EPM tests in CGRP ${ }^{\mathrm{SPFp}}$ (left panel)- or CGRP ${ }^{\mathrm{PBel}}$ neurons (right panel)-silenced mice.

1515 (I, and $\mathbf{J})$ Optogenetic stimulation during hot plate assay $\left(55^{\circ} \mathrm{C}\right)$.

1516 (K, and $\mathbf{L})$ Optogenetic stimulation during electric von Frey assay.

1517 (M) Schematic diagram of optogenetic fear conditioning (photostimulation was used instead of foot shock).

1518 (N) Quantified freezing level $24 \mathrm{hr}$ after optogenetic conditioning.

1519 (O) Schematic diagram of optogenetic cue-dependent fear conditioning (photostimulation paired with 3

$1520 \mathrm{kHz}$ tone).

1521 (P) Context-dependent freezing $24 \mathrm{hr}$ after optogenetic conditioning.

1522 (Q) Cue-dependent freezing $24 \mathrm{hr}$ after optogenetic conditioning.

1523

1524

1525

\section{Statistics}

1526

1527

(B) EYFP: $5.95 \pm 0.33 \mathrm{~s}(\mathrm{n}=15)$, TetTox: $7.82 \pm 0.73 \mathrm{~s}(\mathrm{n}=16)$. Unpaired t test (two-tailed), $\mathrm{p}=0.0306$

1528

(D) EYFP: $4.75 \pm 0.24 \mathrm{~g}(\mathrm{n}=15)$, TetTox: $5.66 \pm 0.33 \mathrm{~g}(\mathrm{n}=16)$. Unpaired $\mathrm{t}$ test (two-tailed), $\mathrm{p}=0.0113$

1529

(F) EYFP: $35.61 \pm 3.18 \%(\mathrm{n}=9)$, TetTox: $23.84 \pm 3.17 \%(\mathrm{n}=8)$. Unpaired t test (two-tailed), $\mathrm{p}=0.0196$

(H) SPFp; EYFP: $26.96 \pm 3.66 \%(\mathrm{n}=7)$, TetTox: $39.83 \pm 4.05 \%(\mathrm{n}=9)$. Unpaired t test (two-tailed), $\mathrm{p}=$ 0.0383 .

1532

PBel; EYFP: $34.56 \pm 7.37 \%(\mathrm{n}=7)$, TetTox: $57.58 \pm 5.15 \%(\mathrm{n}=7)$. Unpaired t test (two-tailed), $\mathrm{p}=$ 0.0250 .

1534

(I) Repeated measure two-way ANOVA showed no significance in interaction $(\mathrm{F}(1,16)=1.79, \mathrm{p}=$ $0.2002)$, Laser $(\mathrm{F}(1,16)=3.36, \mathrm{p}=0.0857)$ and group $(\mathrm{F}(1,16)=1.49, \mathrm{p}=0.2393)$.

(L) Repeated measure two-way ANOVA showed no significance in interaction $(\mathrm{F}(2,36)=1.72, \mathrm{p}=$ $0.1938)$, Laser $(\mathrm{F}(2,36)=0.06, \mathrm{p}=0.9465)$ and group $(\mathrm{F}(1,18)=1.26, \mathrm{p}=0.2768)$. (N) EYFP: $25.31 \pm 2.18 \%(\mathrm{n}=8), \mathrm{ChR} 2: 47.95 \pm 5.42 \%(\mathrm{n}=12)$. Unpaired $\mathrm{t}$ test (two-tailed), $\mathrm{p}=0.0042$. $\mathrm{p}=0.0108), \mathrm{CS}(\mathrm{F}(1,18)=39.57, \mathrm{p}<0.0001)$ and group $(\mathrm{F}(1,18)=6.827, \mathrm{p}=0.0176)$. Freezing at CS- 

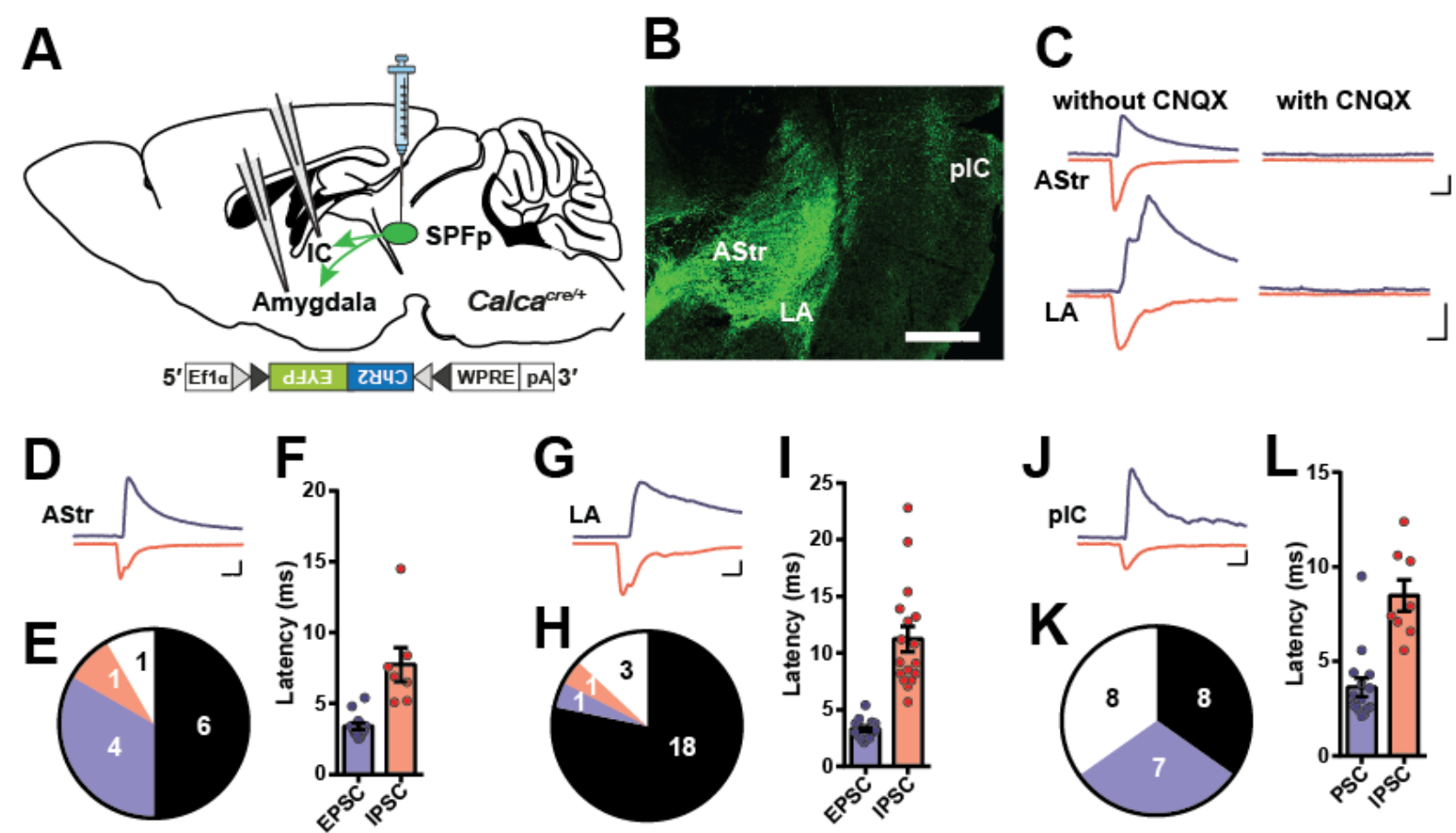

Both $\square$ EPSC only $\square$ IPSC only $\square$ Non Response

M

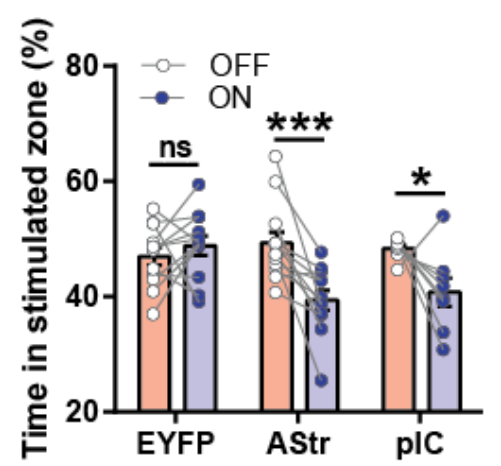

$\mathbf{N}$

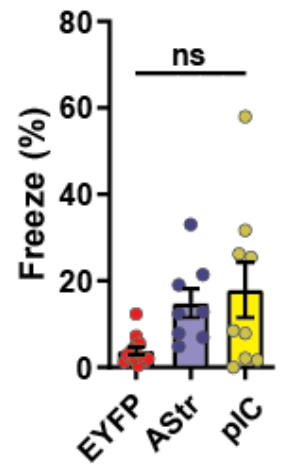

0

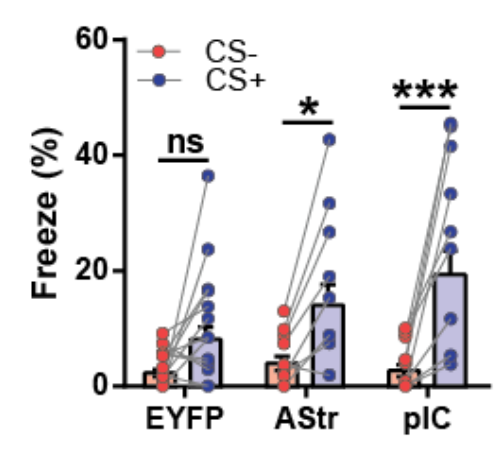

Figure S9. Mapping the functional downstream of CGRP ${ }^{\mathrm{SPF}}$ projection.

1545 (A) Schematics of the experiment.

(B) Representative image of the projection regions from CGRP ${ }^{\mathrm{SPF}}$ neurons. Scale bar indicates $500 \mu \mathrm{m}$.

(C) Example traces of an optically induced EPSC (blue) and IPSC (red) of Astr and LA without or with CNQX to confirm glutamatergic synapse. Scale bars indicate $10 \mathrm{~ms}$ and $50 \mathrm{pA}$.

1549 (D) Examples of Astr EPSC (blue) and IPSC (red) traces by optogenetic terminal activation. Scale bars indicate $10 \mathrm{~ms}$ and $50 \mathrm{pA}$.

1551 (E) Proportion of Astr cells with 'Both' EPSC and IPSC, 'EPSC only', 'IPSC only', or 'No-Response'.

1552

(F) Onset of each Astr EPSC and IPSC to optogenetic stimulation.

(G-L) Results of the same experiments with LA (G-I) and pIC (J-L) neurons. Scale bars indicate $10 \mathrm{~ms}$ 1554 and $50 \mathrm{pA}$.

1555 (M) Result of RTPA with terminal photo-stimulation.

1556 (N) Context-dependent freezing at $24 \mathrm{~h}$ after terminal photo-stimulation conditioning.

1557 (O) Cue-dependent freezing at $24 \mathrm{~h}$ after terminal photo-stimulation conditioning. 


\section{Statistics}

(F) Astr; EPSC: $3.40 \pm 0.25 \mathrm{~ms}(\mathrm{n}=12$ cells), Astr IPSC: $7.74 \pm 1.21 \mathrm{~ms}(\mathrm{n}=7$ cells). Unpaired t test (twotailed), $\mathrm{p}=0.0003$.

1561 (I) LA; EPSC: $3.30 \pm 0.16 \mathrm{~ms}(\mathrm{n}=21$ cells), LA IPSC: $11.24 \pm 1.13 \mathrm{~ms}(\mathrm{n}=17$ cells). Unpaired t test (two1562 tailed), $\mathrm{p}<0.0001$.

1563 (L) pIC; EPSC: $3.63 \pm 0.49 \mathrm{~ms}(\mathrm{n}=15$ cells), IC IPSC: $8.49 \pm 0.83 \mathrm{~ms}(\mathrm{n}=8$ cells). Unpaired $\mathrm{t}$ test (two1564 tailed), $\mathrm{p}<0.0001$.

1565 (M) Repeated measure two-way ANOVA showed significance in laser $\mathrm{X}$ group interaction $(\mathrm{F}(2,29)=$ $15667.215, \mathrm{p}=0.0029)$ and laser $(\mathrm{F}(1,29)=13.24, \mathrm{p}=0.0011$, but not group $(\mathrm{F}(2,29)=2.607, \mathrm{p}=0.0910)$. 1567 Laser ON and was significantly larger than OFF in AStr $(p<0.001)$ and pIC (p < 0.05). Additionally, 1568 difference between EYFP vs Astr $(\mathrm{p}<0.01)$ and EYFP vs pIC $(\mathrm{p}<0.05)$ during ON period were significant. 1569 (N) EYFP: $3.842 \pm 0.89(n=13)$, AStr: $14.82 \pm 3.32(n=8)$, pIC: $17.94 \pm 6.40 \%(n=9)$. One-way ANOVA 1570 showed significant $(\mathrm{p}=0.0161)$. EYFP vs IC was significantly different $(\mathrm{p}<0.05)$ with Tukey's multiple 1571 comparison test.

1572 (O) Repeated measure two-way ANOVA showed significance in CS X group interaction $(\mathrm{F}(2,27)=3.647$, $1573 \mathrm{p}=0.0396)$ and $\mathrm{CS}(\mathrm{F}(1,27)=39.74, \mathrm{p}<0.0001)$ but not in group $(\mathrm{F}(2,27)=2.948, \mathrm{p}=0.0695)$. CS+ was 1574 significantly larger than CS- in AStr $(\mathrm{p}<0.05)$ and $\mathrm{pIC}(\mathrm{p}<0.0001)$. Moreover, difference between EYFP 1575 and $\mathrm{pIC}$ in CS+ was significant $(\mathrm{p}<0.01)$ with Sidak's multiple comparison test. 


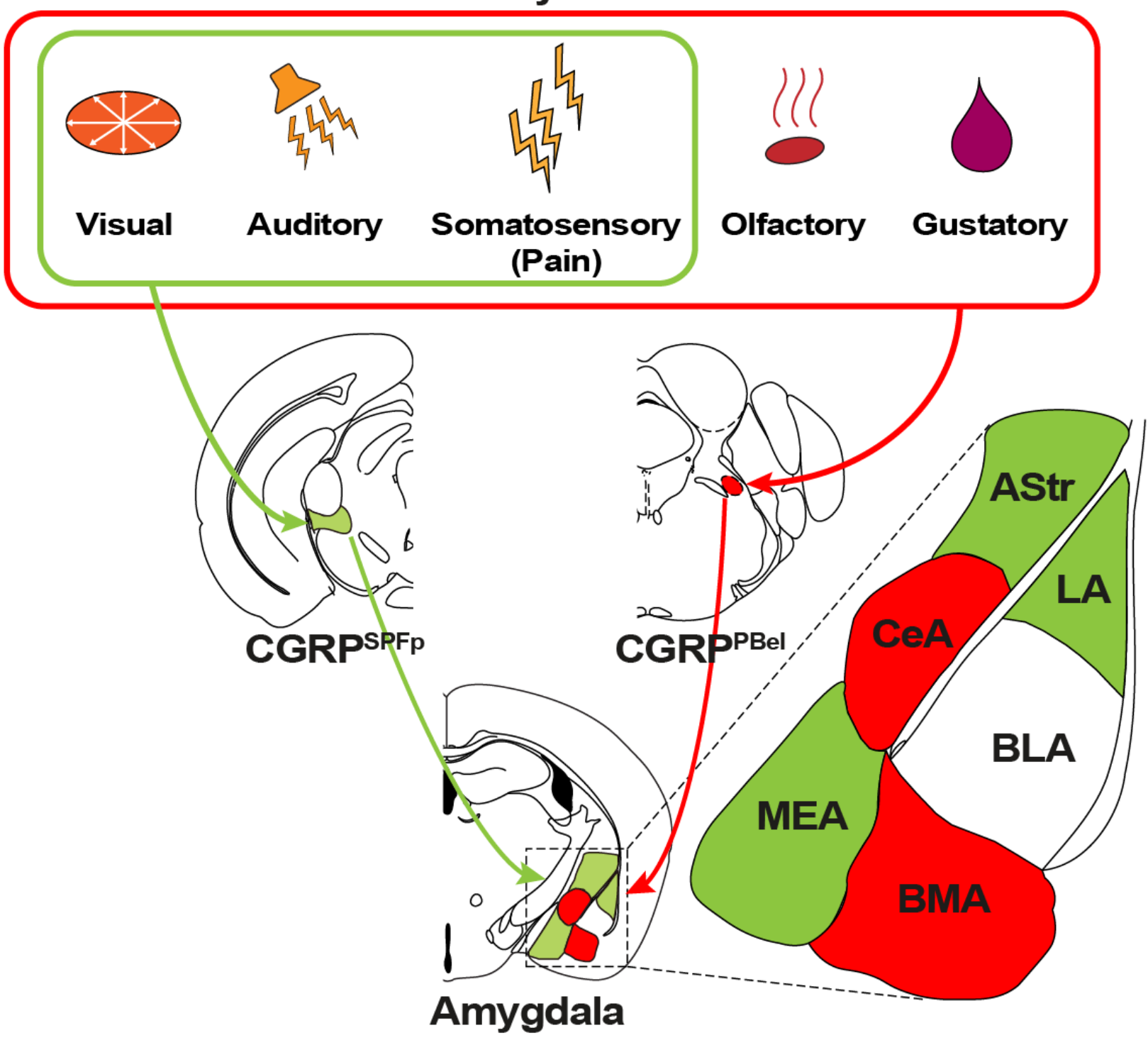

